Aus der Klinik für Anästhesiologie

(Prof. Dr. med. M. Quintel)

im Zentrum Anästhesiologie, Rettungs- und Intensivmedizin

der Medizinischen Fakultät der Universität Göttingen

\title{
Der Einfluss von Crisis-Resource-Management-Training als Teil des medizinischen Curriculums auf die Qualität und Leitlinienadhärenz des Advanced Life Supports
}

\author{
Inaugural - Dissertation \\ zur Erlangung des Doktorgrades \\ der Medizinischen Fakultät \\ der Georg-August-Universität zu Göttingen \\ vorgelegt von \\ Lea Kaminski \\ aus \\ Marburg
}

Göttingen 2015 
Dekan:

I. Berichterstatter:

II. Berichterstatter:

III. Berichterstatter:

Tag der mündlichen Prüfung:
Prof. Dr. rer. nat. H. K. Kroemer

Prof. Dr. med. A. Timmermann

Prof. Dr. med. S. König

Prof. Dr. med. M. Oppermann

12.05.2015 


\section{INHALTSVERZEICHNIS}

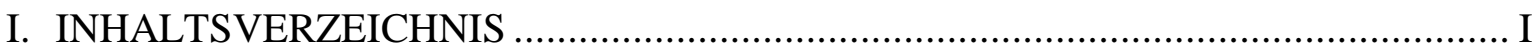

II. ABKÜRZUNGEN, ABBILDUNGEN UND TABELLEN ........................................

II.1 Liste der verwendeten Abkürzungen ..............................................................IV

II.2 Abbildungsverzeichnis .......................................................................... VI

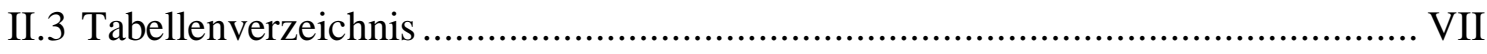

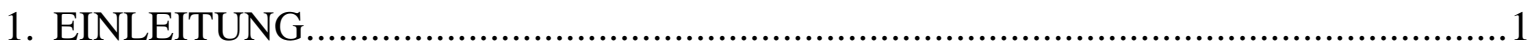

1.1 Geschichtliche Entwicklung der Wiederbelebung ..............................................1

1.2 Advanced Life Support (ALS) - Leitlinien 2005 ................................................

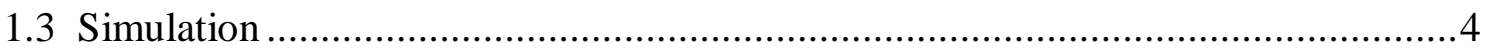

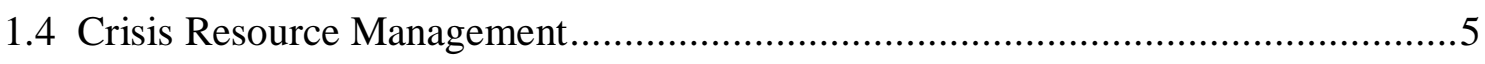

1.4.1 Anwendung von CRM in der Medizin ................................................6

1.4.2 CRM im medizinischen Curriculum .................................................... 8

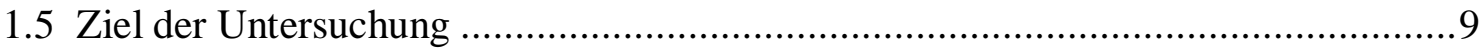

2. MATERIAL UND METHODEN …..................................................................... 10

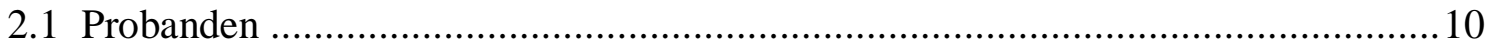

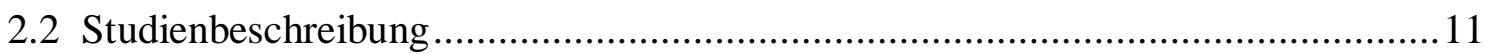

2.2.1 Lehre an der Universität Göttingen: Notfallmedizin................................11

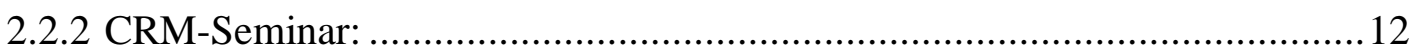

„Die Bewältigung von Zwischenfällen, der 'Human Factor"”................................ 12

2.2.3 Cardiac Arrest Scenario Training (CAST) ............................................. 14

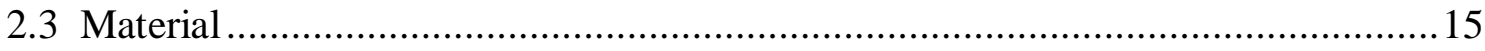

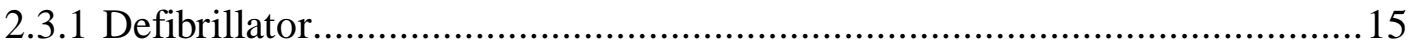

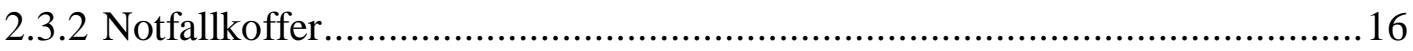

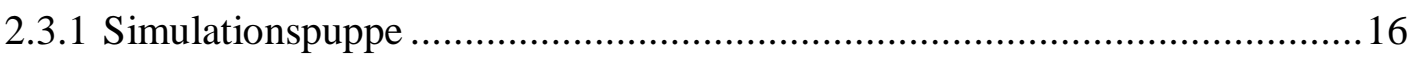

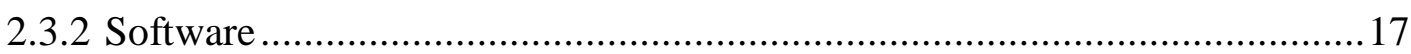

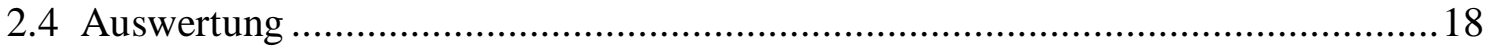

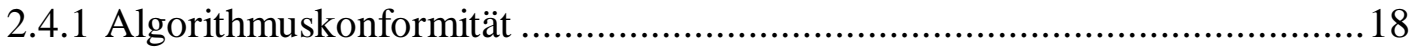

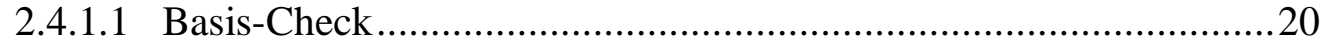

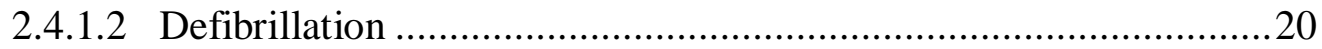

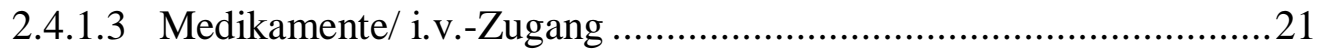

2.4.1.4 Intubation/ asynchrone Beatmung ..........................................2 21

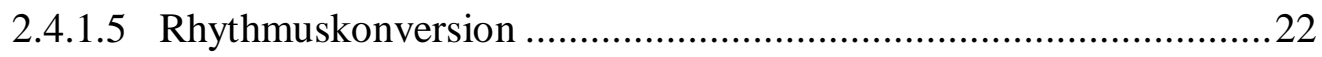

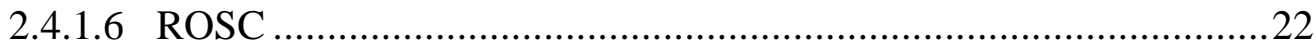


2.4.1.7 Reversible Ursachen ............................................................22

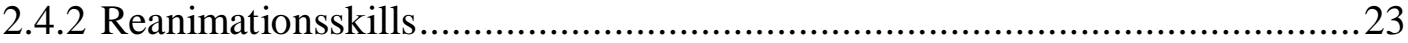

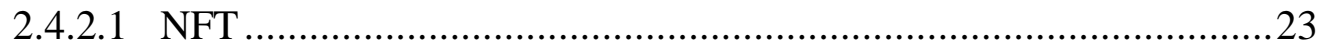

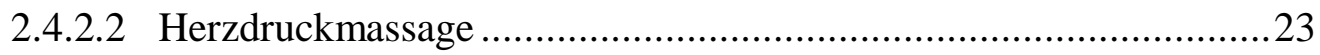

2.4.2.3 Maskenbeatmung ...............................................................24

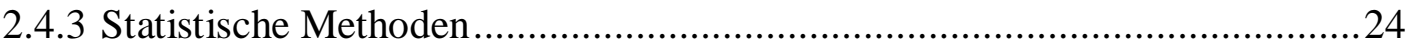

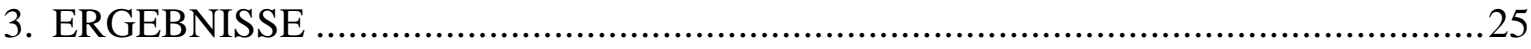

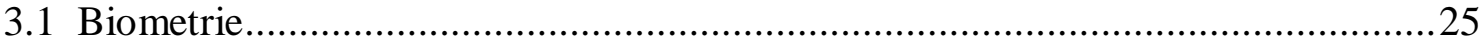

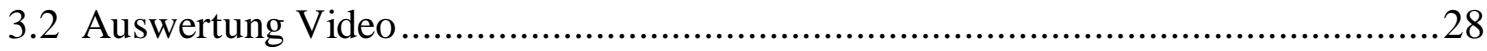

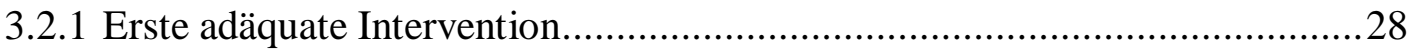

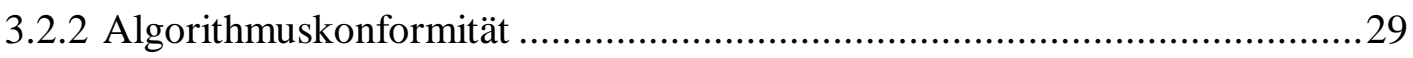

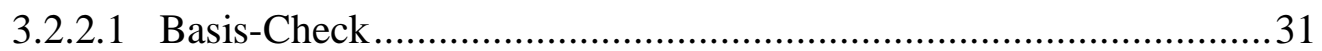

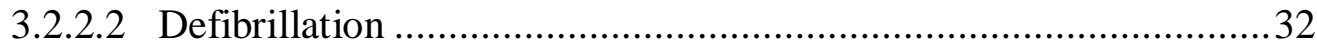

3.2.2.3 Medikamente/ i.v.-Zugang ................................................... 33

3.2.2.4 Intubation/ asynchrone Beatmung .............................................35

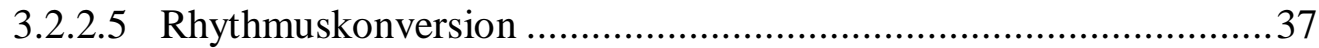

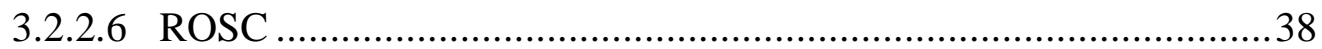

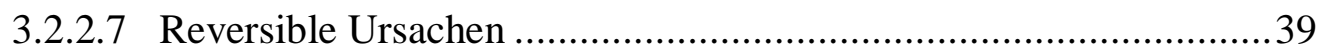

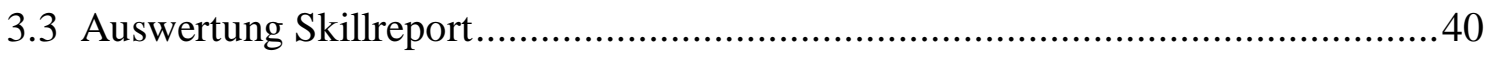

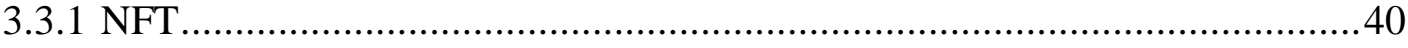

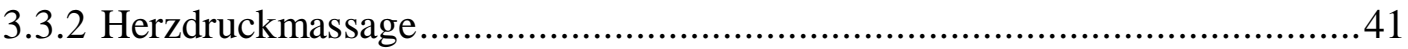

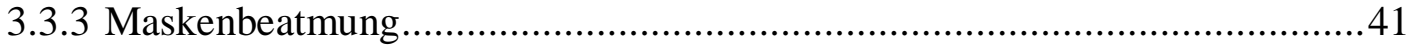

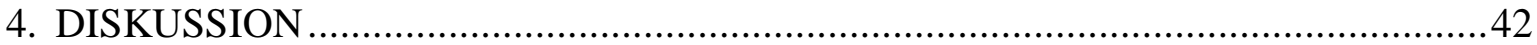

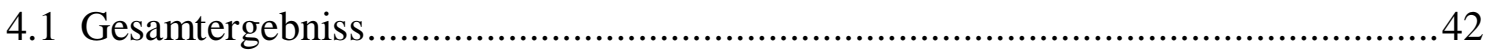

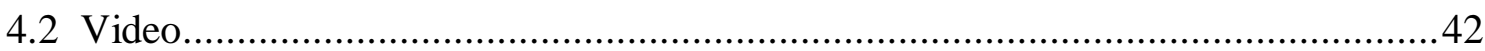

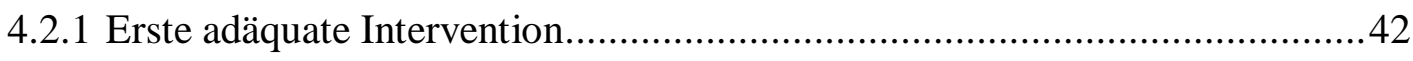

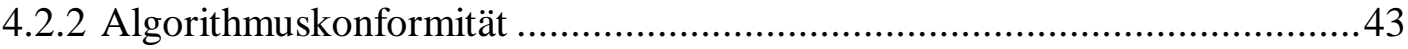

4.2.2.1 Basis-Check .......................................................................44

4.2.2.2 Defibrillation ................................................................. 45

4.2.2.3 Medikamente ........................................................................ 45

4.2.2.4 Intubation/ asynchrone Beatmung ........................................46

4.2.2.5 Rhythmuskonversion, ROSC und reversible Ursachen .................47

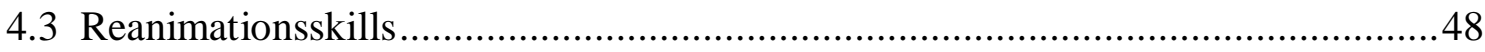

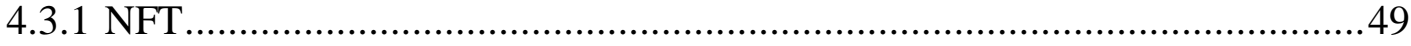




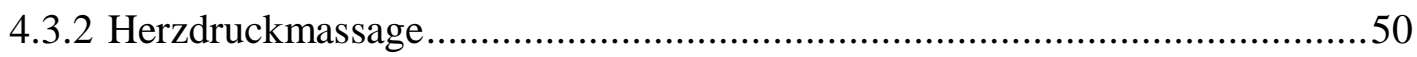

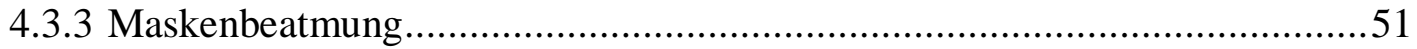

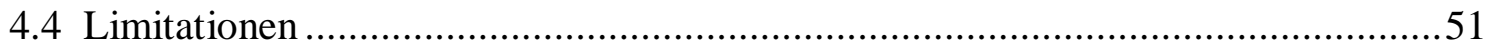

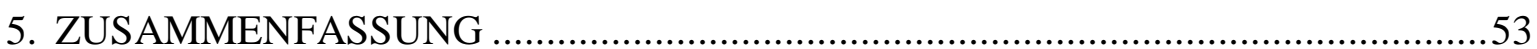

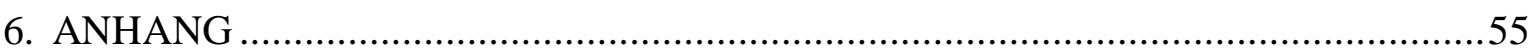

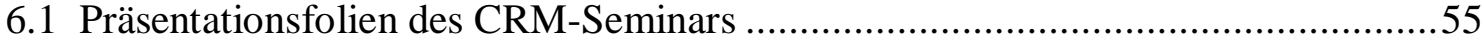

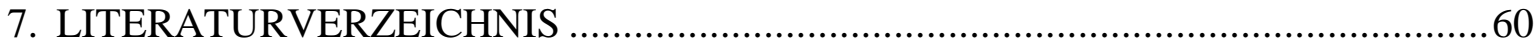


II. Abkürzungen, Abbildungen und Tabellen

\section{II.1 Liste der verwendeten Abkürzungen}

\begin{tabular}{|c|c|}
\hline Abb. & Abbildung \\
\hline ACRM & Anaesthesia Crisis Resource Management \\
\hline AHA & American Heart Association \\
\hline ALS & Advanced Life Support \\
\hline BLS & Basic Life Support \\
\hline bzw. & beziehungsweise \\
\hline ca. & circa \\
\hline CAST & Cardiac Arrest Scenario Training \\
\hline CRM & Crisis Resource Management \\
\hline CPR & cardio pulmonary resuscitation (Kardiopulmonale Reanimation) \\
\hline Defi & Defibrillation \\
\hline EKG & Elektrokardiogramm \\
\hline ERC & European Resuscitation Counsil \\
\hline etc. & et cetera $=$ und so weiter \\
\hline ggf. & gegebenenfalls \\
\hline HDM & Herzdruckmassage \\
\hline IOM & Institute of Medicine \\
\hline i.v. & intravenös \\
\hline MAX & Maximum \\
\hline MB & Maskenbeatmung \\
\hline $\min$ & Minute \\
\hline MIN & Minimum \\
\hline $\mathrm{n}$ & Anzahl \\
\hline NASA & National Aeronautics and Space Administration \\
\hline NAS-NRC & National Academy of Science-National Research Council \\
\hline NFT & no-flow time (Zeit ohne Herzdruckmassage) \\
\hline OSCE & objective structured clinical examination \\
\hline PEA & pulslose elektrische Aktivität \\
\hline ROSC & return of spontaneous circulation \\
\hline SD & Standardabweichung \\
\hline
\end{tabular}




\begin{tabular}{|l|l|}
\hline sek & Sekunde \\
\hline SS & Sommersemester \\
\hline SPSS & Statistical Package for the Social Science (Statistikprogramm) \\
\hline Tab. & Tabelle \\
\hline vs. & versus \\
\hline VF & ventricular fibrillation (Kammerflimmern) \\
\hline VT & ventricular tachycardia (ventrikuläreTachykardie) \\
\hline WS & Wintersemester \\
\hline
\end{tabular}




\section{II.2 Abbildungsverzeichnis}

Abb. 1.2 ALS-Algorithmus nach den ERC-Leitlinien 2005 für Erwachsene (Wenzel et al. 2006) ............................................................................

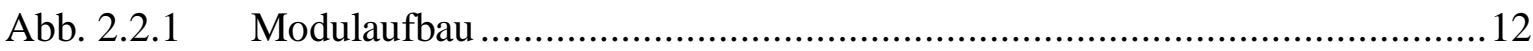

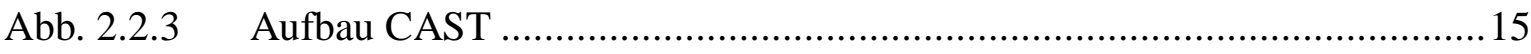

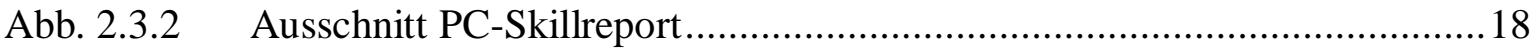

Abb. 3.1.a Flowchart des Probandenkollektivs in der Auswertung Video ..................25

Abb. 3.1.b Flowchart des Probandenkollektivs in der Auswertung PC-Skillreport ......26

Abb. 3.1c Altersverteilung im Auswertungsbereich Video.....................................27

Abb. 3.1.d Altersverteilung im Auswertungsbereich PC-Skillreport...........................27

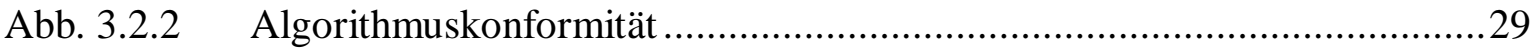

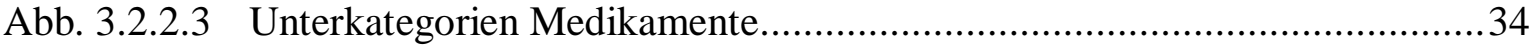

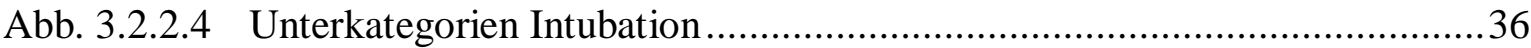

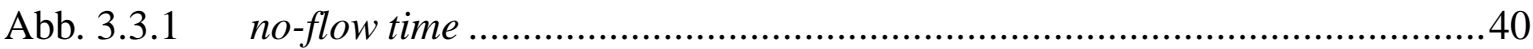




\section{II.3 Tabellenverzeichnis}

Tab. 2.4.1 Punkteverteilung Algorithmuskonformität.............................................19

Tab 3.1 Geschlechterverteilung in den Kohorten ...........................................26

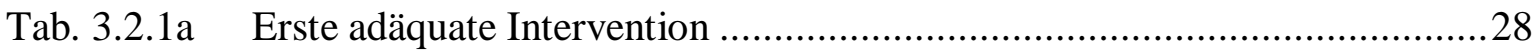

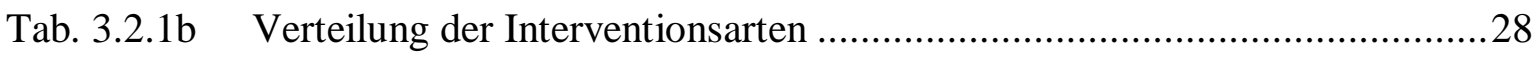

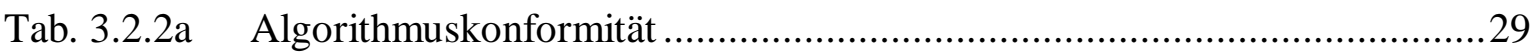

Tab. 3.2.2b Punktzahlen (Median) beider Kohorten in allen Kategorien der

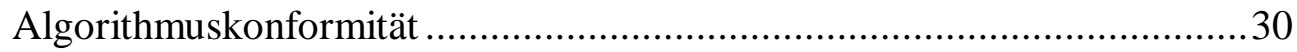

Tab. 3.2.2.1a Gesamtpunktzahl im Basis-Check ...................................................... 31

Tab.3.2.2.1b Unterkategorien des Basis-Checks ........................................................ 31

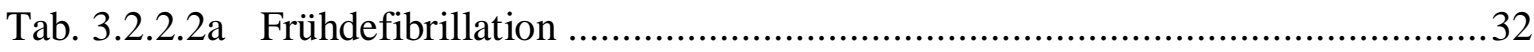

Tab. 3.2.2.2b Unterkategorien der Defibrillation und Gesamtpunktzahl Defibrillation....32

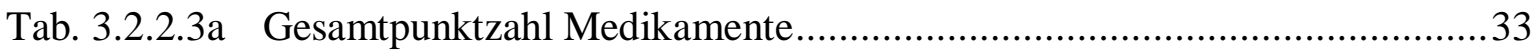

Tab. 3.2.2.3b Unterkategorien Medikamente............................................................... 33

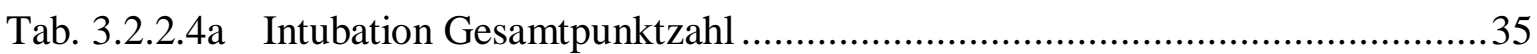

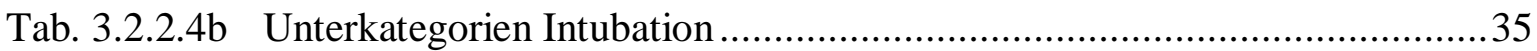

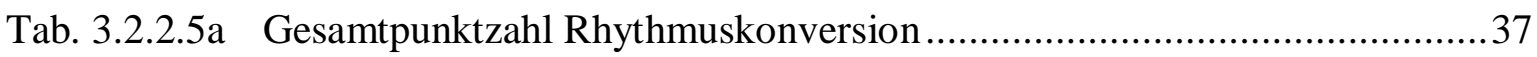

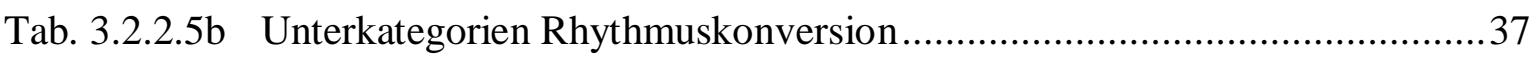

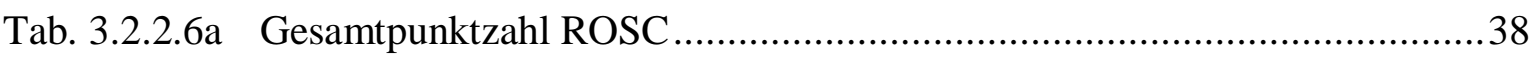

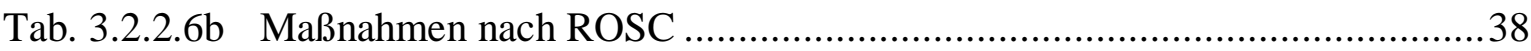

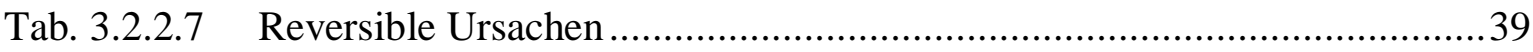

Tab. 3.3.2 Qualität und Quantität der Herzdruckmassage …..................................41

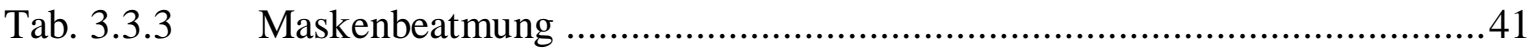




\section{EINLEITUNG}

\subsection{Geschichtliche Entwicklung der Wiederbelebung}

Vor Ende des 19. Jahrhunderts unterschieden sich die Maßnahmen zur Wiederbelebung noch sehr stark von den heutigen, die auf neuen Erkenntnissen beruhen. Leblose Personen wurden kopfüber aufgehängt oder auf rollende Fässer gelegt. Man nahm an, dass sich ihre Lungen durch die passive Thoraxexkursion wieder mit Luft füllen würden (Liss 1986). Die Forschung an effektiveren Methoden begann u.a. mit Experimenten von zunächst interner, dann externer Herzdruckmassage an Hunden im Jahr 1906 (Crile und Dolley 1906). Die Wirksamkeit beider Formen der Herzdruckmassage wurde damit zum ersten Mal wissenschaftlich gesichert. Der Einsatz von externen Defibrillatoren zur Terminierung von malignen Herzrhythmusstörungen gelang 1956 (Zoll et al. 1956). Das Problem der suffizienten Beatmung unter Reanimation löste Elam 1957, der Erfinder der Mund-zuMundbeatmung. Er fand heraus, dass die gespendete Ausatemluft eines Helfers ausreiche, um den Gasaustausch einer leblosen Person aufrechtzuerhalten (Greene et al. 1957). Peter Safar griff die gewonnenen Erkenntnisse der vergangenen Jahre in seiner Entwicklung des ABC-Schemas auf. A (airway) stand für das Freimachen der Atemwege durch Kopfüberstrecken beim Auffinden einer leblosen Person, B (breathing) für die Beatmung und C (circulation/chest compressions) bedeutete Herzdruckmassage (Safar 1999). 1957 veröffentlichte er sein Buch „ABC of resuscitation“. Herzdruckmassage, Defibrillation und Beatmung waren als Grundbausteine der Reanimation erkannt worden und wurden schließlich erfolgreich experimentell kombiniert: 70\% der Patienten mit Kreislaufstillstand überlebten dank dieser Mehode (Kouwenhoven et al. 1960). Um die Reanimationsprinzipien auf der ganzen Welt zu verbreiten und weitergeben zu können, entwickelte Safar nach Veröffentlichung seines Buchs zusammen mit Asmud Laerdal, einem Spielzeughersteller aus Norwegen, die Reanimations-Puppe Resusci Anne zu Übungszwecken. Die heutige Firma Laerdal vetreibt medizinische Lehrausrüstung wie u.a. die genannte Resusci Anne.

Das Thema Reanimation gewann durch die Forschungsergebnisse wachsende Akkzeptanz unter den Medizinern und auch das Interesse der allgemeinen Bevölkerung stieg, sodass in den kommenden Jahren erste Konferenzen zum Verfassen offizieller Empfehlungen zur Reanimation stattfanden (Paraskos 1993). Die erste amerikanische Nationalkonferenz 1966 für kardiopulmonale Reanimation (cardiopulmonary resuscitation=CPR), ausgerichtet von der Academy of Science- National Research Council (NAS-NRC), sprach sich für ein 
einheitliches CPR-Training für medizinisches Personal aus, wofür die Standards von der American Heart Association (AHA) festgelegt werden sollten. Nach der Konferenz wurden die Beschlüsse durch Trainingsprogramme und Materialien realisiert und stark von der AHA und anderen privaten Gruppen gefördert. Daraufhin wurden im Zuge der zweiten Nationalkonferenz 1973, ausgerichtet vom NAS-NRC und der AHA, die vorangegangenen Empfehlungen erweitert. Das CPR-Training sollte nun auf die Allgemeinbevölkerung ausgeweitet werden. Kurse in Basic Life Support (BLS) und Advanced Life Support (ALS), geleitet von geschulten Ausbildern, wurden in das Ausbildungsschema jedes Krankenhauses integriert und Standards zur Durchführung von BLS (Standards CPR 1974b) und ALS (Standards CPR 1974a) von der AHA festgelegt. Leitlinien für BLS und ALS wurden auf der nächsten Nationalkonferenz 1979 entwickelt und 1980 veröffentlicht. Die bisherigen Empfehlungen von 1974 wurden aufgrund fehlender Forschungsergebnisse beibehalten. Hinzu kamen Leitlinien für den pädiatrischen BLS und neonatalen ALS (Standards CPR 1980). Der weltweite Enthusiasmus in der Erforschung der Reanimation stieg, sodass die Empfehlungen 1985 einige Änderungen erfuhren. Durch die Zusammenarbeit der American Acadamy of Pediatrics mit der AHA wurden Aspekte zur Reanimation von Kindern und Neugeborenen in die modifizierten Leitlinien integriert. Desweiteren wurde die zeitige Thrombolyse nach akutem Myokardinfarkt und eine möglichst frühe Defibrillation empfohlen sowie die Ausbildung in primärer und sekundärer Prävention von Kreislaufstillständen und deren Risikoerkennung (Standards CPR 1986).

Zeitgleich zu den akzeptierten Reanimationsentwicklungen der AHA in den USA gab es auch in Europa erste Bemühungen hinsichtlich einheitlicher Reanimation. Auf die Initiative des schwedischen Kardiologen Lars Mogensen trafen sich 1988 unabhängige multidisziplinäre Vertreter verschiedener europäischer Länder in Antwerpen und gründeten den European Resuscitation Counsil (ERC). 1989 wurde er als offizielles Komitee anerkannt. Auf der ersten großen Konferenz des ERCs 1992 waren auch australische und amerikanische Kollegen geladen. Die Vorstellung von einem internationalen Verband für kardiopulmonale Reanimation wurde auf dieser Konferenz mit der Gründung eines Liasion-Komitees etabliert, später bekannt als International Liasion Committee of Resuscitation (ILCOR). Die zugehörigen Unterorganisationen wie u.a. der ERC und die AHA veröffentlichen heute ihre Leitlinien zur Reanimation auf Grundlage des ILCOR-Konsenses.

1992 fand in Amerika die fünfte Nationalkonferenz zur CPR statt. Mittlerweile hatte das Thema eine so große Bedeutung gewonnen, dass zahlreiche Studien seit der letzten 
Konferenz 1985 veröffentlicht wurden, auf deren Erkenntissen nun aufgebaut werden konnte. In den Guidelines fanden die frühzeitige Defibrillation, der Hinweis zur Infektionsgefahr während CPR und der automatisierte externe Defibrillator ihren festen Platz. Desweiteren wurden ethische Gesichtspunkte berücksichtigt (Standards CPR 1992). Um den wissenschaftlichen Wert der Studien zu erhöhen, auf denen die Empfehlungen letztendlich beruhten, mussten Vorgaben zu den Kernpunkten Studiendesign, Methoden, Quellen, Ethik und Umsetzbarkeit des Studiengegenstandes eingehalten werden.

Die Änderungen in den Reanimationsrichtlinien bis zum heutigen Tag belaufen sich auf kleine Details. Der Empfehlungsrahmen für CPR, dessen Grundsteine Anfang des 20. Jahrhunderts gelegt wurden, bleibt jedoch immer erhalten.

Seit dem Jahr 2000 galten in Europa die Leitlinien des ERC, die immer im fünfjährigen Abstand aktualisiert werden. Die vorliegende Arbeit stützt sich auf die Leitlinen von 2005. Die derzeit gültigen Leitlinien wurden 2010 veröffentlicht.

\subsection{Advanced Life Support (ALS) - Leitlinien 2005}

Die Qualität der CPR korreliert positiv mit dem Überleben der Patienten und der Lebensqualität (Wik et al. 1994; Gallagher et al. 1995; Abella et al. 2005). Verspätete und unterbrochene Herzdruckmassage haben eine verminderte koronare und zerebrale Durchblutung zur Folge, beides assoziiert mit abnehmenden Überlebensraten (Valenzuela et al. 2005). Folglich repräsentiert die no-flow time (NFT=Zeit in der keine Herzdruckmassage stattfindet) einen validierten Marker, um CPR-Qualität zu erfassen (Wik et al. 2005). Die ERC-Leitlinien 2005 für Advanced Life Support (Abb 1.2) unterstützen diese Erkenntnisse zur minimalen Unterbrechung der Herzdruckmassage. Alle Änderungen gegenüber den Leitlinien von 2000 zielen auf die möglichst ununterbrochene Herzdruckmassage (Roessler et al. 2008). Sie führten zu einer signifikanten Verbesserung der Reanimationsqualität wie der Reduzierung der NFT mit einem leichten Trend zur Überlebenssteigerung der Patienten bis zur Entlassung (Olasveengen et al. 2009). Auch Ilper et al. konnten zeigen, dass die Leitlinien von 2005 gegenüber 2000 zu einer signifikanten Verringerung der NFT führten (Ilper et al. 2012). Für die vorliegende Arbeit ist vor allem der linke Schenkel des Reanimationsalgorithmus von Bedeutung, der das Handeln im Falle eines Kammerflimmerns bzw. einer ventrikulären Tachykardie beschreibt. 


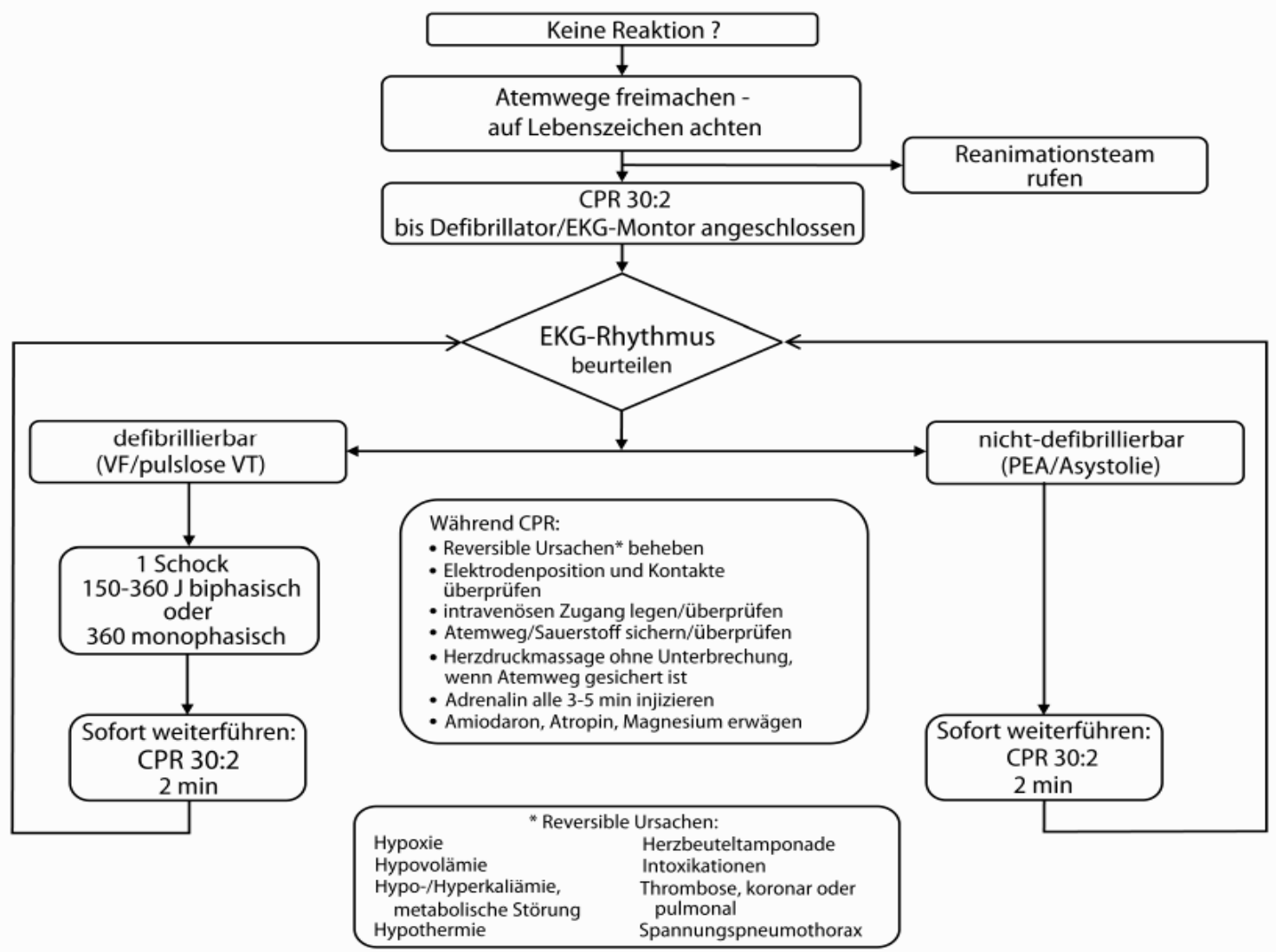

Abb. 1.2 ALS-Algorithmus nach den ERC-Leitlinien 2005 für Erwachsene (Wenzel et al. 2006)

$\mathrm{VF}=$ ventricular fibrillation (Kammerflimmern)

$\mathrm{VT}=$ ventricular tachycardia (ventrikuläre Tachykardie)

$\mathrm{PEA}=$ pulslose elektrische Aktivität

\subsection{Simulation}

Um praktische Fähigkeiten wie ALS zu erwerben, stellt die Simulation eine bewährte und sehr effektive Methode dar (Weller et al. 2004; Ruesseler et al. 2012). Simulation ermöglicht die beliebig wiederholbare Darstellung gleicher Bedingungen zu Übungs- und Forschungszwecken ohne das Risiko, Patienten oder Anwendern zu schaden (Hunt et al. 2007). Sie erleichtert die Umsetzung von kognitiven, psychomotorischen und affektiven Fähigkeiten im klinischen Alltag und hilft, das Verhalten in kritischen Situationen zu verbessern (Timmermann et al. 2005). Durch eine gestiegene Akzeptanz des Übens praktischer Fähigkeiten an Patienten, der Bedeutung von Patientensicherheit und der gesunkenen Toleranz hinsichtlich klinischer Fehler hat die Simulation einen hohen Stellenwert in der medizinischen Lehre erreicht (Okuda et al. 2009). Dennoch existieren vereinzelte Studien, die zeigen, dass Simulation einschüchternd und stressig für die Teilnehmer sein kann (Kurrek und Fish 1996). Somit sollte Simulation angemessen 
verwendet und eine angenehme, sichere Umgebung für die Teilnehmer geschaffen werden (Leblanc 2011).

Es besteht hohe Evidenz für die tatsächliche Übertragung von erlernten Fähigkeiten aus dem Simulationstraining in den klinischen Alltag (Chandra et al. 2008). Auch die Nachhaltigkeit von in simulationsbasierten Trainings vermittelten Inhalten ist höher gegenüber konventionellen Lehrmethoden (z.B. Vorlesung). Assistenzärzte zeigten auch 14 Monate nach einem viermal zweistündigen ALS-Simulationskurs eine stabile Performance (Wayne et al. 2006). Hinsichtlich des ALS-Trainings bildet das Cardiac Arrest Scenario Training (CAST) eine zentrale Komponente und dient zur Übung und Prüfung der erforderlichen Skills (Perkins et al. 2007). In einem CAST wird mit einer Simulationspuppe ein Kreislaufstillstand simuliert, den die Teilnehmer mit entsprechendem Equipment (Defibrillator, Notfallkoffer) zu bewältigen haben.

\subsection{Crisis Resource Management}

Crisis Resource Management (CRM) ist ein Begriff aus der Luftfahrt, dort eher bekannt als Crew Resource Management. Neben der technischen Ausbildung von Luftfahrzeugbesatzungen anhand von Flugsimulation ist auch das Crew Resource Management Training weltweit verpflichtend, das in den achtziger Jahren entwickelt wurde. Hierbei werden nicht-technische Fähigkeiten an Simulatoren trainiert wie u.a. Kommunikation, Entscheidungsfindung, Situationsbewusstsein, Teamführung und Teamarbeit (Helmreich 2000). Diese Schulung in zwischenmenschlichen Fähigkeiten hilft, Fehler und Unfälle aufgrund menschlichen Versagens zu vermeiden. Ursprung eines solchen Trainings bildete eine Untersuchung, basierend auf Daten der National Aeronautics and Space Administration (NASA) 1976-1983, welche ergab, dass mehr als die Hälfte der Flugunfälle durch menschliches Versagen ausgelöst wurden (Billings und Reynard 1984). Die Hauptgründe dafür bestanden aus Kommunikationsfehlern innerhalb der Crew, mangelnder Teamführung und ungenügender Entscheidungsfindung im Cockpit. Auch hierarchische Unterschiede zwischen Befehlsgebenden und untergeordneten Personen mindern die Effektivität von Teams, was ein weiterer Faktor für Unfälle in der Luftfahrt ist (Harris und Li 2008). Wichtig ist, dass das ganze Team in die Diskussion involviert ist, wie man ein bestimmtes Ziel am besten erreichen kann. Das steigert die Effektivität mehr als nur die Anweisungen des Teamleiters alleine (Wilson et al. 2005; Spreier et al. 2006). Das bedeutet, dass Teammitglied zu sein, keine passive Rolle ist. Ein effektives Teammitglied 
muss ein mit den anderen geteiltes Verständnis über die Ziele des Teams haben, muss sich seiner Rolle und Verantwortung bewusst sein, seine Aufgaben priorisieren, Feedback geben, andere Teammitglieder unterstützen und notfalls auch korrigieren (Risser et al. 1999). Klare Kommunikation kann Kommunikationsschwierigkeiten entgegenwirken: der Sender übermittelt eine Nachricht, welche der Empfänger empfängt, interpretiert und den Empfang rückmeldet. Der Sender versichert sich, dass die gesendete Nachricht empfangen wurde. In den klinischen Alltag übersetzt könnte das bedeuten: der Teamleiter fordert ein Teammitglied auf, Adrenalin zu geben, das adressierte Teammitglied rückversichert sich gegebenenfalls über die Dosierung und bestätigt die Gabe (Wilson et al. 2005).

Das CRM-Training zielt mit der Vermittlung von Verhaltensstrategien in Krisen auf die Fehlervermeidung und die Abschwächung von eventuellen Konsequenzen aus Fehlern (Helmreich 2000). Theorie, Gruppenübungen, Diskussionen und eine zu lösende simulierte Krisensituation mit nachfolgender Besprechung (Debriefing) sind die Bausteine des Trainings. Das Debriefing wird von Teilnehmern als wichtigster Teil gewertet, wo Verhaltensalternativen und CRM-Kriterien diskutiert werden, die im Szenario entweder umgesetzt oder missachtet worden sind (Gaba et al. 2001).

\subsubsection{Anwendung von CRM in der Medizin}

1989 wurde erstmalig ein simulationsbasiertes Curriculum für Anästhesisten entworfen, das sich auf die CRM-Prinzipien der Luftfahrt berief (Howard et al. 1992). Das Curriculum wurde Anaesthesia Crisis Resource Management (ACRM) genannt und 1990 bei dem ersten ACRM-Kurs eingesetzt. Das Wort Crew wurde durch Crisis ersetzt, da Howard und Kollegen dies für das Anwendungsgebiet der Anästhesie als passender empfanden. Jede Fachrichtung in der Medizin wurde als Crew betrachtet, bestehend aus einer oder mehreren Personen. Verschiedene Crews müssen zusammen als Team arbeiten, wie z.B. im OP, wo das Team aus der Anästhesie-, Chirurgie- und Pflege-Crew besteht. Mittlerweile hat sich das Kurskonzept auf andere Fachgebiete ausgeweitet und findet hohen Zuspruch bei den Teilnehmern, wie z.B. in der Radiologie (Sica et al. 1999), Notfallmedizin (Reznek et al. 2003) oder Hals-Nasen-Ohren-Heilkunde (Volk et al. 2011).

2000 veröffentlichte das Institute of Medicine (IOM) den aufsehenerregenden Bericht „To err is human: buildung a safer health system“: mindestens 44.000 , wenn nicht sogar 98.000 Menschen sterben in den USA jährlich auf Grund von vermeidbaren medizinischen Behandlungsfehlern. Dafür verantwortliche Vorfälle wie falsche Transfusionen, 
Patientenverwechslung, Operationen an falschen Körperteilen sind Beispiele für zugrundeliegendes menschliches Versagen. Das IOM sprach sich dafür aus, die Zahlen in den nächsten fünf Jahren um 50\% zu reduzieren, alles andere sei unverantwortlich (Kohn et al. 2000). Das durch dieses Buch geförderte Interesse an der Sicherheit von Patienten in Krankenhäusern führte zu einem signifikanten Anstieg von Veröffentlichungen zu diesem Thema (Stelfox et al. 2006). Trotz der gesteigerten wissenschaftlichen Aktivitäten konnten die meisten Krankenhäuser die Vorfälle durch den Faktor Mensch fünf Jahre nach „To err is human“ aber noch nicht halbieren (Morrissey 2004). Zahlen belegen, dass zwar im Krankenhausmanagement mit der Einführung von Warnsystemen für Zwischenfälle reagiert wurde, aber die Möglichkeiten von Teamtrainings im Rahmen des Crisis Resource Managements zum großen Teil ungenutzt blieben. Solche Sicherheitssysteme bieten keine Transparenz für den Faktor Mensch, den eigentlichen Angriffspunkt einer Verbesserungsstrategie zur erhöhten Patientensicherheit (Wischet und Schusterschitz 2009).

Schmidt et al. berichten 2010 über Anwendungen von CRM-Training im Risikomanagement von Krankenhäusern. Zur Erhöhung der Patientensicherheit wurden schon beim Bau eines neuen Krankenhauses in Minden alle Angestellten trainiert, ihre zwischenmenschlicher Fähigkeiten zu verbessern. Die Teilnehmer des CRM-Kurses evaluierten überaus positiv und sahen in ihm einen sehr hohen Nutzen für ihre praktische Tätigkeit (Schmidt et al. 2010).

Einige Berichte über die tatsächlichen Auswirkungen von Teamtraining auf den klinischen Alltag sind in der Literatur zu finden. Es gibt Daten über die schnellere Versorgung von Traumapatienten und die verbesserte Teamleistung (Capella et al. 2010), Fehlerreduzierung und Performanceverbesserung in der Notaufnahme (Morey et al. 2002), Vermeidung vieler Todesfälle und Behinderungen sowie Kosteneinsparungen durch Fehlervermeidung (Risser et al. 1999). In einem amerikanischen Krankenhaus konnten durch CRM-Training Operationen am falschen Körperteil und das Hinterlassen von Operationsmaterial im Patientensitus gesenkt werden. Jedoch konnte dieses Ergebnis ohne wiederholtes Training nicht dauerhaft aufrechterhalten werden (Ricci und Brumsted 2012). 


\subsubsection{CRM im medizinischen Curriculum}

Das CRM-Training hält mehr und mehr Einzug in die Arztausbildung, vor allem in die Anästhesie seit dem ursprünglichen ACRM-Konzept von Howard et al. 1992. CRM-Kurse für Ärzte und Medizindstudenten erhalten hohen Zuspruch und werden als sehr hilfreich und wichtig evaluiert. Teilnehmer geben an, das Gelernte 6-12 Monate nach dem CRMKurs im Kliniksalltag tatsächlich angewendet zu haben (Flanagan et al. 2004). Zu ähnlichen Ergebnissen kam auch die Klinik für Anästhesiologie des Universitätsklinikums Aachen 2009. Krüger et al. zeigten durch das CRM-Training als Wahlfachangebot für Medizinstudenten einen hohen Lerneffekt bezüglich Kommunikation und effektiven Teamworks sowie eines Zuwachses an subjektivem Sicherheitsgefühl im Management kritischer Situationen im Kliniksalltag (Kruger et al. 2009). Ein einheitliches Curriculum für den Unterricht von CRM für Studierende gebe es bisher nicht, bemängeln Lerner et al. Sie fordern dringlich die Etablierung der Teamworkausbildung in das medizinische Curriculum im Konsens mit der in ihrem Review betrachteten Literatur (Lerner et al. 2009).

Ein Vergleich hinsichtlich der CPR-Leistungen zwischen Allgemeinärzten und Medizinstudenten im letzten Jahr ergaben eine längere NFT und eine spätere, erste adäquate Intervention, Defibrillation und Herzdruckmassage unter den Studierenden. Auch hier verlangen die Autoren nach einer speziellen Ausbildung in den nicht-technischen Fähigkeiten während des Studiums, um die angehenden Ärzte besser auf die bevorstehende Verantwortung in Notfallsituationen während ihrer ersten Arbeitserfahrungen vorzubereiten (Luscher et al. 2010). Für die Umsetzung dieser Ziele ist die Kombination aus CRM und Simulation unerlässlich (Gaba et al. 2001). 


\subsection{Ziel der Untersuchung}

Crisis Resource Management ist eine neue Lehrmethode in der Ausbildung von Medizinstudenten. Ihre positiven Effekte auf Leistungen im Team sind in der Literatur nur in einigen Aspekten beschrieben. Im Anbetracht der Forderungen für die Implementierung des CRM in das medizinische Curriculum sollen Ziele dieser Arbeit sein:

- die Integration eines CRM-Seminars mit Lehrvideos in das Curriculum der Notfallmedizin der Universität Göttingen zu beschreiben;

- den Einfluss des CRM-Seminars auf die Leistungen von Medizinstudenten in den erweiterten Maßnahmen zur Wiederbelebung (ALS) im Vergleich mit den Leistungen der Studierenden ohne vorheriges CRM-Seminar hinsichtlich der Reanimationsqualität (unterbrochene Herzdruckmassage (NFT), erste adäquate Intervention, Herzdruckmassage und Beatmung) und der Einhaltung der Reanimationsleitlinien (Algorithmuskonformität) mit Hilfe eines neu entwickelten Punktescores zu untersuchen. 


\section{MATERIAL und METHODEN}

\subsection{Probanden}

Aus Gründen der Vereinfachung wird in dieser Arbeit, wenn von Personen gesprochen wird, ausschließlich die männliche Form verwendet. Personen weiblichen wie männlichen Geschlechts sind darin gleichermaßen eingeschlossen.

Die prospektive, doppelblinde, randomisierte und simulationsbasierte Fall-/Kontrollstudie fand im Rahmen des zweiwöchigen curriculären Kurses für Notfallmedizin am Lehr- und Simulationszentrum für Anästhesiologie, Rettungs- und Intensivmedizin der GeorgAugust-Universität in Göttingen statt. Zugelassene Teilnehmer dieses Kurses bildeten Studierende der Humanmedizin im zehnten Fachsemester. Von der Studie ausgeschlossen wurden Studierende mit Erfahrungen im Rettungsdienst (Rettungssanitäter/-assistenten). Es wurde sichergestellt, dass nur Studierende an der Studie teilnahmen die die Praktika zum Thema Basic Life Support (BLS), Airwaymanagement, Defibrillation und Rhythmusanalyse zuvor besucht hatten. Im Praktikum Basic Life Support wurde das Vorgehen als Ersthelfer beim Auffinden einer leblosen Person anhand eines Reanimationstorsos geübt, der Resusci Anne Advanced Skilltrainer (C) (Firma Laerdal, Stavanger, Norwegen). Die Übung umfasste das Ansprechen der am Boden liegenden „Person“, Hilfe rufen, Überprüfen und Freimachen der Atemwege, Notruf absetzen und die korrekte Durchführung von Herzdruckmassage und Mund-zu-Mund-Beatmung im Verhältnis 30:2. Das Praktikum Airwaymanagement beinhaltete das Üben der Maskenbeatmung sowie das Kennenlernen und Anwenden verschiedener Methoden zur Atemwegssicherung (Guedel-/Wendltubus, endotrachealer Tubus, Larynxmaske, Intubationslarynxmaske). Dazu wurde der Airway Management Trainer (C) (Firma Laerdal, Stavanger, Norwegen) genutzt. Weiterhin wurde der korrekte und sichere Umgang mit dem Defibrillator (Kap. 2.3.1) in einem Praktikum demonstriert und geübt sowie das Erkennen verschiedener Herzrhythmusstörungen erlernt wie u.a. das Kammerflimmern.

Der Studienzeitraum erstreckte sich über zwei aufeinanderfolgende Semester: Wintersemester 2009/10 und Sommersemester 2010. Insgesamt nahmen 156 Studierende an der Studie teil. Sie wurden alphabetisch von in 39 Gruppen $(n=39)$ aufgeteilt zu jeweils vier Personen. Die Gruppen wurden randomisiert (computergeneriert) den Kohorten zugeordnet: 23 Gruppen nahmen vor der Datenerhebung an einem CRM-Training teil (Interventionsgruppe) und 16 Gruppen an einem ALS-Skill-Training (Kontrollgruppen) 
(Abb. 3.1.a und b). Im Folgenden werden zur Vereinfachung die Interventionsgruppen als CRM-Gruppen und die Kontrollgruppen als Skillgruppen bezeichnet. Alle teilnehmenden Studierenden erklärten sich mit ihrer Unterschrift einverstanden für Studienzwecke gefilmt zu werden und waren sich ihrer Kohortenzugehörigkeit nicht bewusst.

\subsection{Studienbeschreibung}

\subsubsection{Lehre an der Universität Göttingen: Notfallmedizin}

Innerhalb von zwei Wochen durchliefen die Göttinger Medizinstudenten den Lehrplan der Notfallmedizin, aufgeteilt in Vorlesungen, Praktika und Seminare mit einer abschließenden mündlich-praktischen Prüfung an fünf Stationen (OSCE=objective structured clinical examination) sowie einer Klausur (Abb. 2.2.1).

Relevante Kursbausteine für die Untersuchung bildete zunächst die vorbereitende Vorlesung zum Thema ALS und BLS nach den Leitlinien des ERC 2005. Hierbei wurde der BLS-Algorithmus kurz wiederholt, der im 5. Fachsemester bereits vermittelt, praktisch geübt und geprüft wurde. Eine ausführliche theoretische Einführung in ALS mit Hinweisen zu den Elementen Herzdruckmassage, Defibrillation, Rhythmusanalyse und Medikamentenapplikation folgte, um die theoretischen Grundlagen der Studierenden für die praktischen Übungen zu sichern.

Die praktischen Übungseinheiten bildeten vier obligatorische Notfallpraktika, wobei zunächst jene zum Thema BLS, Airwaymanagement und Defibrillation/Rhythmusanalyse (Kap.2.1) absolviert wurden, bevor die Studierenden im vierten Praktikum alle erlernten Fähigkeiten mit Hilfe des Anne Advanced Skilltrainer (C) in einem CAST übertragen und anwenden mussten. Dies bedeutete eine vollständige Reanimation bei beobachtetem Kreislaufstillstand mit Kammerflimmern im vierköpfigen Team.

Die Studierenden der Fallgruppen nahmen vor dem CAST an einem interaktiven, videobasierten Seminar zum Thema CRM teil, in dem die CRM-Prinzipien vorgestellt, abermals der ALS-Algorithmus wiederholt und beides in Zusammenhang gebracht wurde (siehe 2.2.2). Die Studierenden der Kontrollgruppen hingegen absolvierten vor dem CAST ein Skilltraining, in dem auch der ALS-Algorithmus wiederholt, jedoch kein Inhalt zu CRM vermittelt wurde. Danach konnten alle Inhalte der Praktika (Defibrillation, Rhythmusanalyse, BLS, Airwaymanagement) nochmals praktisch an den Geräten und Puppen geübt werden, unter der Anleitung eines Dozenten. 
Nach dem CAST nahmen die Gruppen an der jeweils fehlenden Unterrichtseinheit teil, die Fallgruppen am Skilltraining und die Kontrollgruppen am CRM-Seminar. Somit wurde das dort vermittelte Wissen und die Trainingsmöglichkeiten allen Studierende gewährt, um keine Nachteile zwischen den Gruppen hinsichtlich der Abschlussprüfung (OSCE) zu erzeugen.

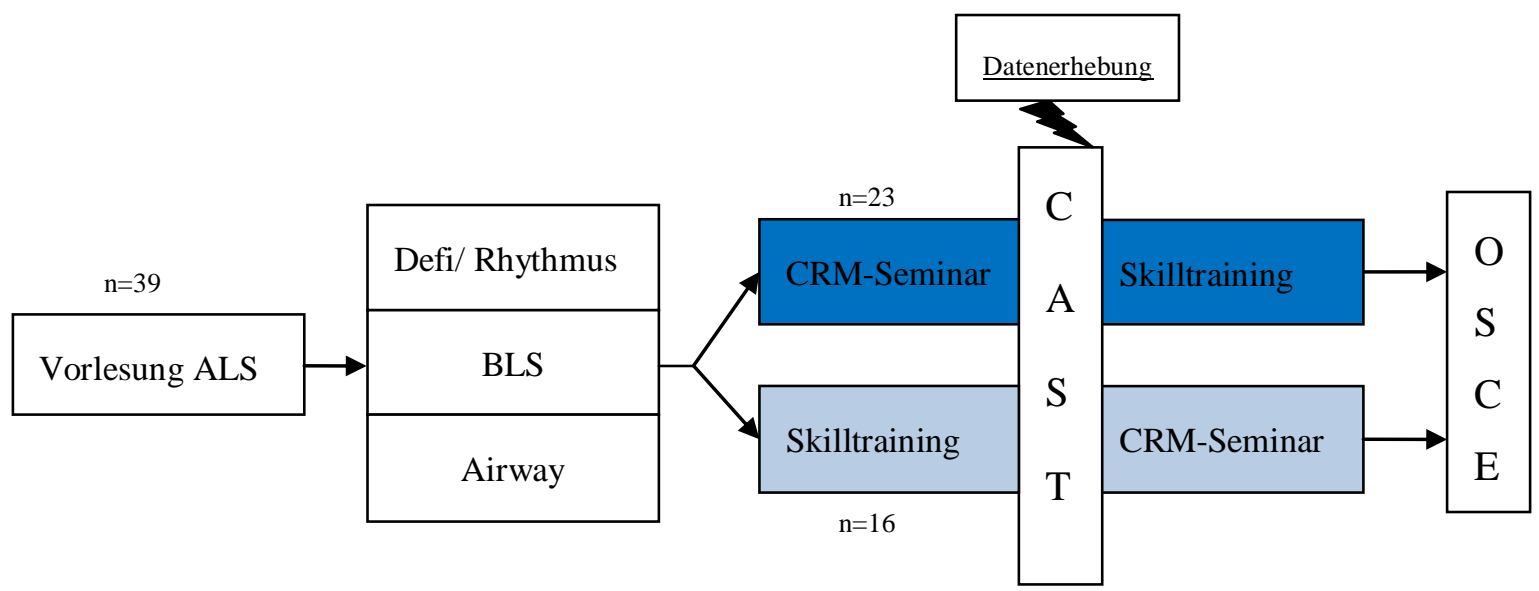

Abb. 2.2.1 Modulaufbau

Fallgruppen (CRM)

Kontrollgruppen (Skill)

\subsubsection{CRM-Seminar:}

\section{„Die Bewältigung von Zwischenfällen, der 'Human Factor'”}

In dieser Unterrichtseinheit (90 Minuten) wurde der Algorithmus zum ALS kurz wiederholt und eine sinnvolle Aufgabenverteilung innerhalb eines Reanimationsteams anhand eines Planspieles erarbeitet. Ziel war es zu erkennen, dass es einen Teamleiter geben muss, der die Reanimation leitet, ohne selbst in praktische Tätigkeiten involviert zu sein. Der Teamleiter übernimmt das Zeitmanagement, um z.B. Rhythmusanalysen zum korrekten Zeitpunkt durchführen zu lassen. Desweiteren muss er Anweisungen geben und in Rücksprache mit dem Team Entscheidungen treffen. Idealerweise sollte ein Teammitglied für Herzdruckmassage und Defibrillation, einer für Atemwegsmanagment und einer für Medikamente und „Hilfe“ zuständig sein. Der Aspekt „Hilfe“ umfasst u.a. die Vorbereitung für eine Intubation oder das Ablösen bei der Herzdruckmassage. Im Falle von kleinen Reanimationsteams, wie im CAST mit vier Personen, lassen sich die Aufgabenfelder sinnvoll miteinander kombinieren, sodass eine mögliche Aufgabenverteilung eines Reanimationsteams folgendermaßen aussehen könnte 
1.Teammitglied: Teamleiter

2.Teammitglied: Basis-Check, Atemwegsmanagement

3.Teammitglied: Defibrillation, Herzdruckmassage

4.Teammitglied: Medikamente, Hilfe

Anhand von Checklisten wurden die Aufgaben genau definiert (siehe Anhang). Die verantwortliche Person für beispielsweise das Atemwegsmanagement muss den initialen Basis-Check durchführen, zweimal nach jeder 30.Thoraxkompressionen mit Hilfe von Beutel und Maske beatmen, sich die Intubation vorbereiten lassen und diese unter laufender HDM versuchen durchzuführen, mit anschließender Lagekontrolle. Sie muss auf die Maskenbeatmung bei Fehlintubation zurückgreifen bzw. bei gelungener Intubation mit einer Frequenz von 10-12/min asynchron, d.h. unter durchgehender HDM, ventilieren. Rückmeldungen über durchgeführte Tätigkeiten waren für jedes Teammitglied obligativ an den Teamleiter zu richten.

Nach dem medizinischen Teil des Seminares, wurde zum kommunikationspsychologischen Teil mit Hilfe von Katastrophenbeispielen aus der Schifffahrt übergeleitet, die auf menschliche Fehler zurückzuführen waren, auf den sogenannten „Human Factor“. Übertragen auf die Medizin bedeuten das z.B. Todesfälle oder Folgeschäden durch Behandlungsfehler, denn 75\% aller Zwischenfälle in der Anästhesie sind auf den Faktor Mensch zurückzuführen (Chopra et al. 1992). Als Lösungsvorschlag wurden die CRMKriterien als nicht-technische Fähigkeiten vorgestellt, als Hilfe für einen Arzt, um Krisen nicht nur auf der Basis von medizinischem Wissen zu bewältigen. Diese umfassten Kommunikation, Teambildung/-leitung, Antizipation und Planung, Nutzung von Resourcen und Reevaluation.

Nach diesen theoretischen Einführungen wurden zwei Lehrvideos von simulierten Reanimationen gezeigt, die es von den Studierenden zu analysieren galt: zunächst eine „schlechte“ und dann eine „sehr gute“ Reanimation, gespielt von vier Mitarbeitern des Lehr- und Simulationszentrums Göttingen. Anhand des ersten Videos sollten die Studierenden die medizinischen und die nicht-technischen Fehler diskutieren. Es folgte die Sequenz der sehr guten Reanimation, in der beispielhaft der ALS-Algorithmus angewandt und viele CRM-Prinzipien umgesetzt wurden, die mit Overlays an den entsprechenden Szenen sichtbar gemacht worden waren. 
Abschließend wurde das zweite Video ohne Overlays abermals gezeigt und die Studierenden erhielten die Aufgabe, die Verwendung von CRM-Prinzipien spielerisch anhand eines Bingos aufzuzeigen. Jeder erhielt eine Karte mit unterschiedlichen CRMAspekten, die es schnellstmöglich im Video zu identifizieren galt.

\subsubsection{Cardiac Arrest Scenario Training (CAST)}

Auf die drei Praktika und das CRM-Seminar im Falle der Interventionsgruppen bzw. des Skilltrainings bei den Kontrollgruppenmitgliedern folgte das $\mathrm{zu}$ untersuchende vierte Praktikum CAST. Die Dozenten, die das CAST betreuten, erhielten zuvor eine Einführung in den Ablauf und die Technik. Diese lag auch in schriftlicher Form zur Erinnerung vor dem Praktikum für die Dozenten im Praktikumsraum bereit. Das CAST bestand aus zwei Szenarien, wobei nur das erste zu Studienzwecken analysiert wurde, um den Lerneffekt zu vermeiden. Im Folgenden wird der Begriff CAST auch nur im Sinne des ersten Szenarios verwendet. Genutzt wurde ein Resusci Anne Advanced Skilltrainer (C) als Patientensimulator, womit Aufzeichnungen der Reanimationsdaten über das entsprechende PCSkillreporting System (C) (Firma Laerdal, Stavanger, Norwegen; Version 2.2.1, 2003) auf einem Laptop gemacht und gespeichert wurden. Zur späteren Videoanalyse wurde das erste Szenario jeder Gruppe auch in Bild und Ton aufgezeichnet. Die Puppe lag auf dem Boden und war über ein Kabel mit der Steuerung zum Einspielen des erwünschten Herzrhythmus und dem Laptop verbunden. Notfallkoffer und Defibrillator wurden von dem vierköpfigen „Reanimationsteam“ zum Einsatzort mitgebracht.

Vor Beginn des ersten Szenarios erhielten die Studierenden eine kurze Einführung in die Funktionen der Simulationspuppe und den Inhalt des Notfallkoffers sowie eine Beschreibung der Ausgangssituation vor der Tür: die vier Studierenden im ersten Szenario seien Ärzte auf einer gefäßchirurgischen Station. Während der morgendlichen Visite käme eine Schwester hilferufend $\mathrm{zu}$ ihnen und berichtete, dass Herr Meier während des Waschens soeben kollabiert sei. Mit Notfallkoffer und Defibrillator ausgestattet betrat das Team das „Patientenzimmer“ und das Szenario begann.

Gruppen, die vorher das CRM-Seminar besucht hatten, bekamen vor Beginn des Szenarios die Checklisten mit der Aufgabenverteilung ausgehändigt: Teamleiter, HDM und Defi, Medikamente und Hilfe, Airwaymanagement. Nach der Teambildung und kurzer Wiederholung der jeweiligen Zuständigkeiten mussten die Checklisten wieder abgegeben werden, bevor das Szenario begann. Die Kontrollgruppen erhielten die gleiche Zeit vor 
dem Szenario, sich als Team zu besprechen, jedoch ohne CRM-Vorkenntnisse aus dem noch für sie anstehenden Seminar und ohne die Hilfe von Checklisten.

Der initiale Herzrhythmus des „Patienten“ war stets Kammerflimmern. Nach vier Defibrillationen konvertierte der Rhythmus in einen Sinusrhythmus, auch genannt return of sponateous circulation (ROSC). Den Teilnehmern wurde dann ausreichend Zeit gegeben, die HDM für zwei Minuten fortzuführen und anschließende Maßnahmen zu bedenken (post-resuscitation-care). Damit endete das Szenario und eine Besprechung folgte.

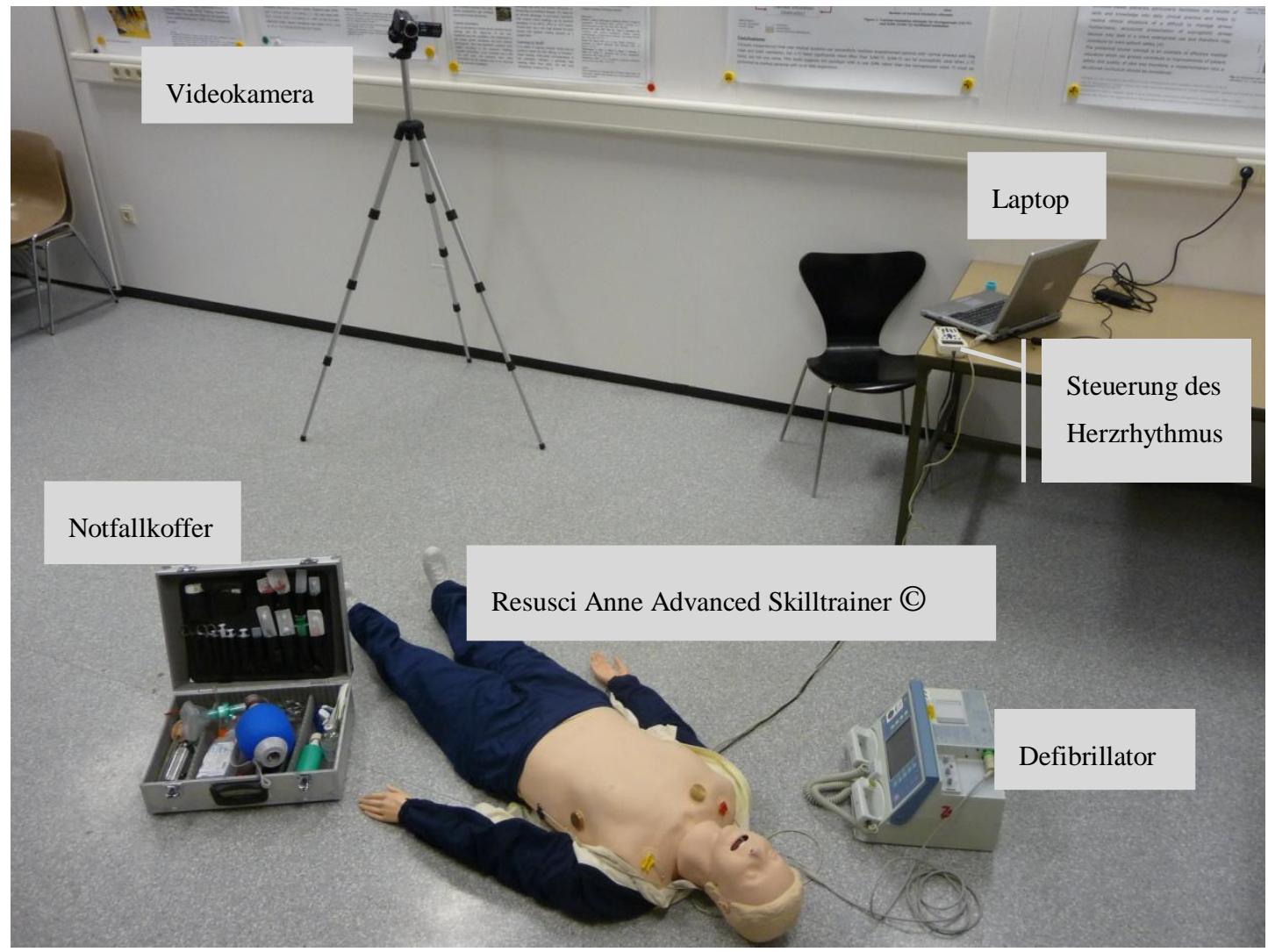

Abb. 2.2.3 Aufbau CAST

\subsection{Material}

\subsubsection{Defibrillator}


Während des Moduls wurde ausschließlich der monophasische Defibrillator Bruker 2000 (C) (Bruker Medical Cardio GmbH, Ettlingen, Deutschland) verwendet.

Die Studierenden erhielten eine Einweisung zum sicheren Gebrauch im Praktikum und übten das Defibrillieren mit den drei Kommandos „Achtung Defibrillation“ (Herausnahme der Paddels aus dem Gerät und Positionierung auf dem Thorax der Puppe), „Achtung, ich lade, alle weg vom Patienten“ (vor dem Laden auf 360 Joule) und „Achtung, ich schocke“ (nach dem Laden, aber vor der Schockabgabe). Parallel zu den letzten beiden Kommandos galt es, sich sorgfältig zu vergewissern, dass kein Teammitglied körperlichen Kontakt zur Puppe hatte.

\subsubsection{Notfallkoffer}

Der zur Verfügung stehende Notfallkoffer enthielt das notwendige Equipment, um das Szenario leitliniengerecht zu lösen. Materialien für das Airwaymanagement bildeten eine Puritanmaske, Beatmungsbeutel, Beatmungsfilter, Gesichtsmaske, Guedeltubus, Wendltubus, Laryngoskop, Tubus der Größe 7.0, Führungsstab, Magillzange, Larynxmaske der Größe 3 und ein Stethoskop. Zur i.v.-Applikation der bereitstehenden Medikamente Adrenalin, Amiodaron und Atropin standen Venenverweilkanülen, Stauschlauch, Pflaster, Spritzen, Kanülen, NaCl-Lösung und Desinfektionsmittel zur Verfügung. Als Infusion wurde eine zuvor geleerte 500ml-Elektrolytlösung mit angeschlossenem Infusionssystem verwendet, um versehentliches Auslaufen im Koffer und auf der Puppe zu vermeiden.

Darüber hinaus befand sich im Notfallkoffer eine Blutdruckmanschette.

\subsubsection{Simulationspuppe}

Der lebensgroße Reanimationstrainer Resusci Anne Advanced Skilltrainer (C) stellte im CAST den zu versorgenden „Patienten“ dar, der Atmung nicht simulieren, jedoch einen Karotispuls generieren konnte. Über zwei Metallplatten ließ sich mit einem Defibrillator das EKG ableiten und im Falle auch defibrillieren. Der Herzrhythmus wurde über eine Steuerung eingestellt, wobei zwischen vielen verschiedenen Rhythmen ausgewählt und die Pulsstärke reguliert werden konnte. In diesem Fall waren jedoch nur Kammerflimmern und Sinusrhythmus von Bedeutung. Die Fernbedienung war mit der Puppe über ein Kabel verbunden, um eventuelle Störungen von parallel genutzten Puppen im Nebenzimmer zu minimieren. Zusätzlich konnten zur Überwachung des Herzrhythmus auch EKGElektroden an der Puppe angeschlossen werden. Die Puppe besaß anatomisch 
nachgebildete Atemwege, was die Maskenbeatmung und endotracheale Intubation ermöglichte. Zur Atemwegssicherung konnten auch alternative Maßnahmen, wie z.B. die Larynxmaske angewendet werden. Der linke Arm der Puppe bot die Möglichkeit zur Venenpunktion, jedoch wurde diese zur Schonung des Materials im Praktikum nur durch Aufkleben des Gefäßzugangs angedeutet. Auch die Medikamente wurden nicht tatsächlich appliziert und statt dessen nur Luft in die Spritze aufgezogen.

\subsubsection{Software}

Über das PC-Skillreporting System (C) konnten die Daten der Reanimation visualisiert und gespeichert werden. Zur Vereinfachung wurde im Folgenden der Begriff PC-Skillreport verwendet. Aufgezeichnet wurde die Durchführung der Herzdruckmassage (Drucktiefe, Entlastung, Handposition) und der Beatmung (Atemzugvolumen). Der laufende Herzrhythmus wurde angezeigt sowie der Zeitpunkt des Defibrillierens. Darüber hinaus wurden Aktionen wie das Freimachen der Atemwege und das Tasten des Karotispuls registriert und auf der fortlaufenden Zeitachse angezeigt. Die Kompressionsfrequenz wurde per Hand ausgezählt wie auch die NFT. 


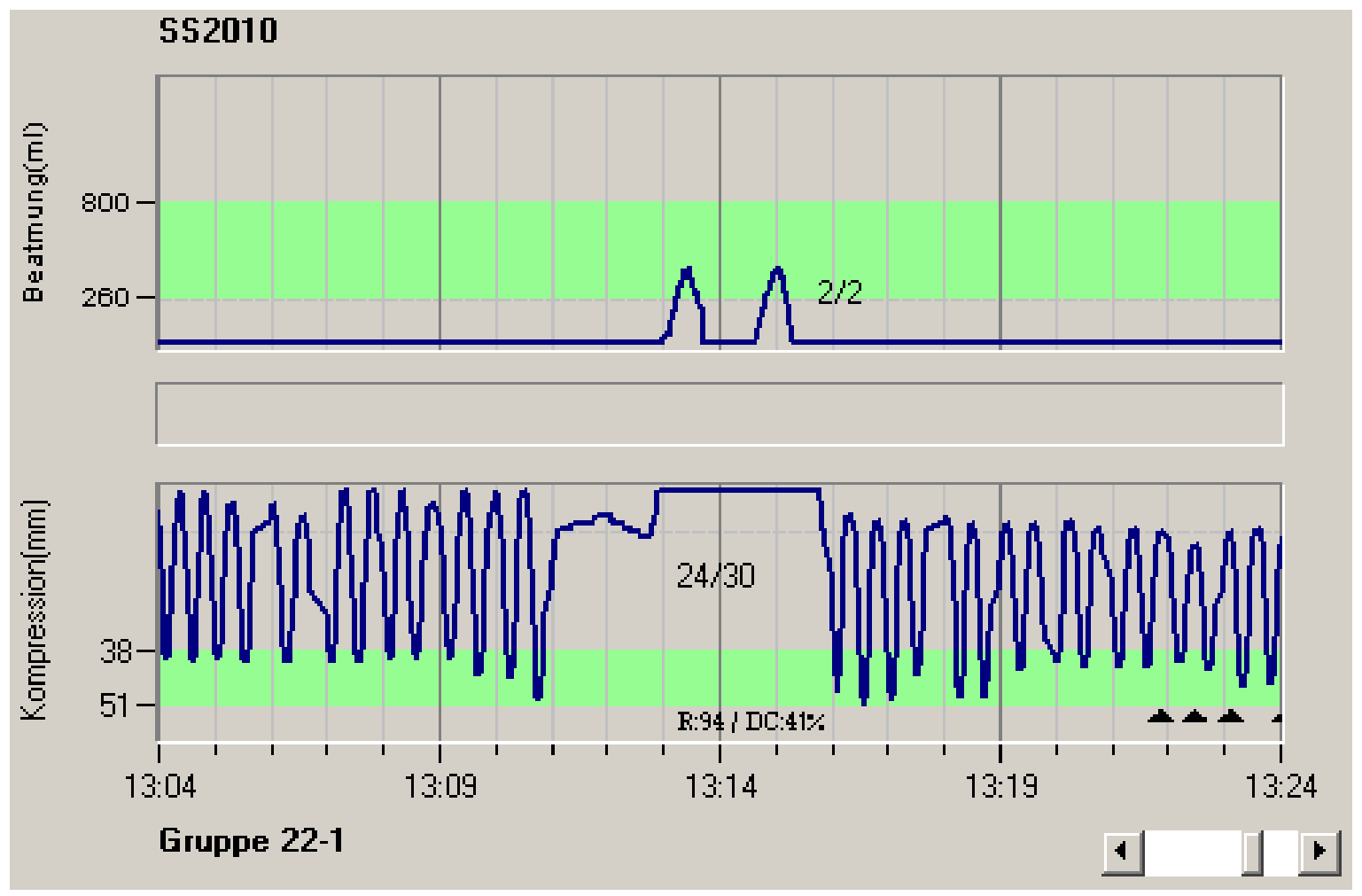

Abb. 2.3.2 Ausschnitt PC-Skillreport

: $\quad$ korrektes Beatmungsvolumen bzw. Kompressionstiefe

24/30: $\quad 24$ korrekte Kompressionen hinsichtlich Tiefe, Entlastung und Handposition von 30 durchgeführten Kompressionen

2/2: $\quad$ beide Beatmungen korrekt hinsichtlich Volumen und Geschwindigkeit durchgeführt

- : $\quad$ unvollständige Entlastung

\subsection{Auswertung}

\subsubsection{Algorithmuskonformität}

Der folgende Abschnitt beschreibt einen entwickelten Punktescore zur objektiven Beurteilung von Reanimationen hinsichtlich ihrer Konformität zum ALS-Algorithmus der ERC-Leitlinien 2005. An der Entwicklung beteiligten sich ERC-Instruktoren des Lehr- und Simulationszentrums Anästhesiologie, Rettungs- und Intensivmedizin in Göttingen.

Der Punktescore (Tab. 2.4.1) wurde auf beobachtete Kreislaufstillstände mit defibrillierbaren Rhythmen ausgelegt und erfasste Szenarien, die nach vier Defibrillationen zum ROSC führten. Die aufgezeichneten Videos während des CASTs, wurden anhand dieser Checkliste bewertet. Der maximal erreichbare Wert betrug 60 Punkte. 


\begin{tabular}{|c|c|c|c|}
\hline Kategorie & Unterkategorie & Punkte & Punkte insgesamt \\
\hline \multirow[t]{4}{*}{ Basis-Check } & Ansprechen & 1 & 4 \\
\hline & Atmung & 1 & \\
\hline & Pulskontrolle & 1 & \\
\hline & Diagnose Herz-Kreislaufstillstand & 1 & \\
\hline \multirow[t]{3}{*}{ 1. Defi } & indizierte Durchführung & 1 & 8 \\
\hline & sicher $^{1}$ und mit Gel durchgeführt & 1 & \\
\hline & als Frühdefibrillation ${ }^{2}$ & 6 & \\
\hline \multirow[t]{4}{*}{ 2. Defi } & indizierte Durchführung & 1 & 4 \\
\hline & Zeitintervall HDM 2min +/- 30sek & 1 & \\
\hline & $+/-15 \mathrm{sek}$ & 2 & \\
\hline & sicher & 1 & \\
\hline \multirow[t]{4}{*}{ 3. Defi } & indizierte Durchführung & 1 & 4 \\
\hline & Zeitintervall HDM 2min +/- 30sek & 1 & \\
\hline & $+/-15$ sek & 2 & \\
\hline & sicher & 1 & \\
\hline \multirow[t]{4}{*}{ 4. Defi } & indizierte Durchführung & 1 & 4 \\
\hline & Zeitintervall HDM 2min +/- 30sek & 1 & \\
\hline & $+/-15$ sek & 2 & \\
\hline & sicher & 1 & \\
\hline i.v. Zugang & ja & 2 & 2 \\
\hline \multirow[t]{2}{*}{ Adrenalin } & gegeben & 2 & 4 \\
\hline & zwischen 2. und 3.Schock & 2 & \\
\hline \multirow[t]{2}{*}{ Amiodaron } & gegeben & 2 & 4 \\
\hline & zwischen 3. und 4.Schock & 2 & \\
\hline \multirow[t]{2}{*}{ Intubation } & versucht & 2 & 4 \\
\hline & Lagekontrolle $^{3}$ & 2 & \\
\hline HDM/Beatmung & kontinuierliche HDM nach Intubation & 5 & 5 \\
\hline \multirow[t]{4}{*}{ Rhythmuskonversion } & erkannt & 2 & 10 \\
\hline & weiter HDM & 4 & \\
\hline & weiter HDM für $2 \mathrm{~min}$ & 4 & \\
\hline & Abbruch HDM nach Rhythmuskonversion & -8 & \\
\hline \multirow[t]{3}{*}{ ROSC } & Pulskontrolle zentral & 2 & 6 \\
\hline & Blutdruckkontrolle & 2 & \\
\hline & post-resuscitation-care diskutiert & 2 & \\
\hline \multirow[t]{2}{*}{ reversible Ursachen } & diskutiert & 1 & 1 \\
\hline & & & 60 \\
\hline
\end{tabular}

Tab. 2.4.1 Punkteverteilung Algorithmuskonformität

${ }^{1}$ Mindestens ein Kommando „Sicherheit“ (dazu 2.1.4.2) und kein Kontakt zur Puppe, geladene Paddels nicht in der Luft

2 Durchführung der ersten Defibrillation innerhalb 90 Sekunden nach Betreten des Raumes

${ }^{3}$ Auskultation beidseits und/oder $\mathrm{CO}_{2}$ angefordert und/oder Ansage „unter Sicht“ intubiert 


\subsubsection{Basis-Check}

Die erste Maßnahme beim Auffinden einer leblosen Person stellt der Basis-Check dar. Dieser beinhaltet das laute Ansprechen der Person mit Körperkontakt (1 Punkt) und die Überprüfung der Atmung (1 Punkt). Das Freimachen der Atemwege wurde hierbei nicht bewertet, da dies anhand des Videos nicht eindeutig zu beurteilen war. Die Kreislaufkontrolle wird vom ERC für den Laien nicht ausdrücklich empfohlen, dennoch ist das Tasten des Karotispulses (1 Punkt) von professionellen Helfern zu erwarten (Wenzel et al. 2006). Das Stellen einer Diagnose ist für den Beginn jeder Therapie unverzichtbar. In diesem Falle war es das Verbalisieren der Diagnose Herz-Kreislaufstillstand in Form von „wir müssen reanimieren“ oder ähnlichen Äußerungen (1 Punkt), was den unverzüglichen Beginn der Therapie (Reanimation) einleitete. Für die Kategorie Basis-Check konnten insgesamt vier Punkte erreicht werden.

\subsubsection{Defibrillation}

Für jede Defibrillation war grundlegend ein Punkt für ihre Durchführung zu erlangen, sofern ein defibrillierbarer Rhythmus vorlag und die Defibrillation somit indiziert war.

War die erste Defibrillation eine Frühdefibrillation, wurden sechs Punkte vergeben. Eine Frühdefibrillation wurde definiert durch eine Schockabgabe von professionellen Helfern spätestens 90 Sekunden nach Erreichen des leblosen Patienten. Erfolgte die erste Defibrillation später als 90 Sekunden, da z.B. zunächst mit der Herzdruckmassage begonnen wurde bis die Defibrillation vorbereitet war, dann wurde diese regulär nur mit einem Punkt für ihre indizierte Durchführung bewertet.

Hiermit sollte die Dringlichkeit der sofortigen Defibrillation bei Kammerflimmern hervorgehoben und belohnt werden.

Eine korrekt bzw. sicher durchgeführte Defibrilllation (1 Punkt) beinhaltete die Verwendung von Elektrodengel vor dem ersten Schock, die Verbalisierung von mindestens einem Kommando zum Thema Sicherheit („Achtung, ich lade“, „alle weg“ etc.), der Nachweis (Video), dass zum Defibrillationszeitpunkt kein Körperkontakt zum Patienten bestand sowie die Positionierung von geladenen Paddels entweder im Gerät oder auf dem Patienten. Die Einstellung der korrekten Joulezahl konnte in der Beurteilung nicht berücksichtigt werden, da es in den meisten Fällen nicht möglich war, sie retrospektiv anhand des Videos zu ermitteln. 
Desweiteren wurden Punkte bezüglich des Defibrillationsintervalls verteilt, das gemäß den Leitlinien zwei Minuten betragen sollte. Definiert wird dieses Intervall vom Moment der Wiederaufnahme der Herzdruckmassage nach einem Schock bis zur nächsten Rhythmuskontrolle (Nolan et al. 2005). Je genauer die zwei Minuten eingehalten wurden, desto mehr Punkte wurden vergeben. Betrug das Intervall zwei Minuten +/- 30 Sekunden gab es einen Punkt, bei zwei Minuten +/- 15 Sekunden zwei Punkte. Lag das Intervall außerhalb dieser Toleranzgrenzen, konnte kein Punkt für die Unterkategorie „Intervall“ erlangt werden. Maximal konnten also pro Defibrillation für die Durchführung, korrektes Intervall und ausreichende Sicherheit vier Punkte verteilt werden, für die erste Defibrillation sogar acht Punkte, wenn diese früh genug erfolgte, indiziert und sicher war.

\subsubsection{Medikamente/ i.v.-Zugang}

Bei persistierendem Kammerflimmern empfehlen die ERC-Leitlinien 2005 u.a. die Gabe von Adrenalin und Amiodaron (Nolan et al. 2005). Die intravenöse oder endotracheale Applikation von Adrenalin (2 Punkte) sollte initial zwischen der zweiten und dritten Defibrillation erfolgen (2 Punkte), die intravenöse Gabe von Amiodaron (2 Punkte) zwischen der dritten und vierten Defibrillation (2 Punkte). Mit der korrekten Medikamentengabe, auch hinsichtlich des empfohlenen Zeitpunktes der Gabe, konnten insgesamt acht Punkte erworben werden.

Das Legen einer Venenverweilkanüle als Voraussetzung für die intravenöse Medikamentenapplikation und Volumengabe wurde insgesamt mit zwei Punkten bewertet.

\subsubsection{Intubation/ asynchrone Beatmung}

Da die Leitlinien die Intubation unter Reanimation von geübten Helfern zwar befürworten, aber keinesfalls ausdrücklich empfehlen, sollte auch hier der Fokus nicht auf die Intubation gelegt werden. Somit sind im Abschnitt Intubation maximal vier Punkte zu erwerben. Der Intubationsversuch als solcher wurde mit zwei Punkten gewichtet. Im Fall einer endotrachealen Intubation zählte die folgende Lagekontrolle des Tubus ebenfalls zwei Punkte. Beidseitiges Auskultieren und/oder $\mathrm{CO}_{2}$-Messung bzw. Anforderung und/oder die Intubation unter Sicht galten als durchgeführte Lagekontrolle.

Die benötigte Zeit für die Intubation blieb unberücksichtigt, da diese bei der Auswertung der Skills über die NFT und Ventilationsdaten bereits erfasst wurde. 


\subsubsection{Rhythmuskonversion}

Die vierte Defibrillation führt in den Szenarien zum ROSC und war somit immer erfolgreich. Zur Kreislaufsicherung musste die Herzdruckmassage dennoch fortgeführt werden (4 Punkte) und zwar über einen Zeitraum von zwei Minuten (4 Punkte). Erst danach folgte die Rhythmusanalyse, in der das Erkennen, dass kein Kammerflimmern mehr vorlag, mit zwei Punkten entlohnt wurde. Falls die Herzdruckmassage nach der vierten Defibrillation abgebrochen wurde, da z.B. sofort die Konversion in einen Sinusrhythmus erkannt wurde, hatte das einen Punktabzug von acht Punkten zur Folge. Maximal konnten in der Kategorie Rhythmuskonversion also plus zehn Punkte erreicht werden, minimal durch den CPR-Abbruch jedoch sogar minus acht Punkte.

\subsubsection{ROSC}

Eine Pulskontrolle (2 Punkte) und Blutdruckmessung (2 Punkte) sollten sich nach den erforderlichen zwei Minuten HDM nach Rhythmuskonversion anschließen und auch das weitere Vorgehen (post-resuscitation-care) sollte von den Helfern diskutiert werden (2 Punkte), wie z.B. das Verlegen des Patienten auf Intensivstation etc. Für die Messung des Blutdrucks musste die Manschette angelegt werden, woraufhin der Wert von dem betreuenden Dozenten angesagt wurde. Insgesamt wurden für dieses Verhalten sechs Punkte vergeben.

\subsubsection{Reversible Ursachen}

Reversible Ursachen sollten unter laufender CPR besprochen (1 Punkt) und wenn möglich behoben werden. Dazu zählen die vier H's (Hypoxie, Hypothermie, Hypovolämie, Hypo/Hyperkaliämie) und die HITS's (Herzbeuteltamponade, Intoxikation, Thromboembolie, Spannungspneumothorax). Die Aufzählung der möglichen Ursachen musste nicht vollständig sein, sie sollten lediglich bedacht und diskutiert werden. 


\subsubsection{Reanimationsskills}

Die Erfassung der Reanimationsskills erfolgte anhand des PC-Skillreporting Systems@. Die automatisch errechneten Leistungsdaten wie NFT und Kompressionsfrequenz wurden bei der Auswertung nicht berücksichtigt, da die Werte über die ganze Länge des aufgezeichneten Szenarios vom Programm gemittelt wurden. Da es durch den betreuenden Dozenten im CAST nicht gewährleistet werden konnte, dass das Programm exakt synchron zum Geschehen gestartet und beendet wurde, waren die automatisch ermittelten Werte unzuverlässig und konnten nicht verwendet werden. Die zu messenden Parameter wurden deshalb alle manuell anhand der Graphen (Abb. 2.3.2) ausgezählt. Um die Reanimationsleistungen der Gruppen unabhängig von der Länge des Szenarios miteinander vergleichen zu können, wurden die Leistungen NFT, HDM und Ventilation pro Minute ausgezählt, addiert und der Mittelwert gebildet.

Beginn der ersten Minute stellte die erste adäquate Intervention dar. Als erste adäquate Intervention galt die Defibrillation, Thoraxkompression oder Ventilation (Situation eines beobachteten Kreislaufstillstandes). Ausgewertet wurde nun in einem 1-Minutenraster bis zum Auslösen der vierten Defibrillation, wobei die dafür angebrochene Minute, in der die vierte Defibrillation durchgeführt wurde, nicht mehr berücksichtigt wurde. Für jede Minute wurden Daten zu den folgenden Parametern ausgezählt:

\subsubsection{NFT}

Die NFT entspricht der Zeit (Sekunden pro Minute), in der keine Herzdruckmassage durchgeführt, d.h. in der kein künstlicher Kreislauf aufrecht erhalten wurde wie während der Rhythmusanalyse, Defibrillation und Maskenbeatmung. Angegeben wird die NFT in dieser Arbeit in Prozent. Das heisst, in wieviel Prozent der Zeit, in der HDM durchgeführt werden sollte, dies nicht erfolgte. Startpunkt hierfür ist die erste adäquate Intervention und Endpunkt die vierte Defibrillation.

\subsubsection{Herzdruckmassage}

Neben der Frequenz pro Minute wurde die durchschnittlich korrekte Drucktiefe $(3,8$ $5,1 \mathrm{~cm}$ ), Handposition und vollständige Entlastung, jeweils als prozentualer Anteil der durchgeführten Kompressionen, angegeben. Das Programm zeigte für jede durchgeführte Kompression an, ob diese ausreichend tief (im grünen Bereich), mit falscher Handposition (Handsymbol) oder unvollständiger Entlastung (Dreieck) durchgeführt wurde (siehe Abb. 
2.3.2). Die Kompressionen wurden nur bis zum Start der ggf. asynchronen Beatmung nach erfolgreicher Intubation ausgezählt, da die Puppe das gleichzeitige Ventilieren und Komprimieren des Thorax jeweils nicht korrekt registrieren konnte und das Programm einen falschen Graphen zeichnete.

\subsubsection{Maskenbeatmung}

Es wurde die prozentuale Anzahl korrekter Maskenbeatmungen (MB) erhoben.

$$
\% \text { korrekte } \mathrm{MB}=\frac{\text { korrekte } \mathrm{MB} / \mathrm{min}}{\text { durchgeführte } \mathrm{MB} / \mathrm{min}}
$$

Tidalvolumina zwischen 260 und 800ml wurden als korrekte Ventilationen gewertet, da sich die Puppe im Vergleich zum Menschen schwerer beatmen lässt, d.h. ein normales Tidalvolumen von 500ml durch die Studierende selten erreicht wurde. Die Beatmungen nach Intubation wurden nicht ausgewertet, da sich diese, wie in Kapitel 2.4.2.2 beschrieben, nicht korrekt darstellen ließen.

\subsubsection{Statistische Methoden}

Die Daten wurden mit Hilfe der Statistikprogramme SPSS (C) (Firma IBM, Armonk, New York, Vereingte Staaten; Version 19) und STATISTICA (C) (Firma StatSoft, Tulsa, Oklahoma, Vereinigte Staaten; Version 10) analysiert. Zur Untersuchung möglicher Unterschiede in den Leistungen der geschulten und nicht geschulten Gruppe wurde ein unverbundener T-Test oder ein nichtparametrischer Test (Mann-Whithney-U-Test) verwendet. Weiterhin wurde zum Vergleich ordinaler Daten ein Chi-Quadrat-Test durchgeführt. Für alle statistischen Tests wurde ein Signifikanzniveau von $\alpha=0,05$ festgelegt. 


\section{ERGEBNISSE}

\subsection{Biometrie}

Die Untersuchung fand im Notfallmedizinmodul des WS 2009/10 und SS 2010 statt. Insgesamt nahmen ursprünglich 216 Studierende an der Studie teil, aufgeteilt in 54 Gruppen zu jeweils vier Personen. Diese 54 Gruppen wurden randomisiert zu 27 Kontrollgruppen (Skill) und 27 Interventionsgruppen (CRM). Im Laufe der Module mussten jedoch einige Gruppen ausscheiden, da sie die Studienbedingungen aus verschiedenen Gründen nicht erfüllten: neun Gruppen der Kontrollkohorte (Skill) nahmen durch Fehlorganisation erst nach dem CAST am Skilltraining teil; frühzeitiger Szenarienabbruch durch den betreuenden Dozenten im CAST; Umspringen des Herzrhythmus durch Fehlfunktion der Puppe; Gruppenzusammenschluss aus zwei Gruppen, aufgrund von Nichterscheinens einiger Studierende; frühzeitiges Auftreten von ROSC nach der dritten Defibrillation; fehlende oder unvollständige Daten aus dem PCSkillreport aufgrund technischer Fehler oder durch vergessenes Starten des Programms durch den Dozenten.

Da für die Auswertung der Algorithmuskonformität bzw. ersten adäquaten Intervention die Videoaufnahmen und für die Auswertung der Reanimationsskills der PC-Skillreport genutzt wurden, unterscheiden sich die Gruppenanzahlen der beiden Auswertungsbereiche (Abb. 3.1.1 und 3.1.2).

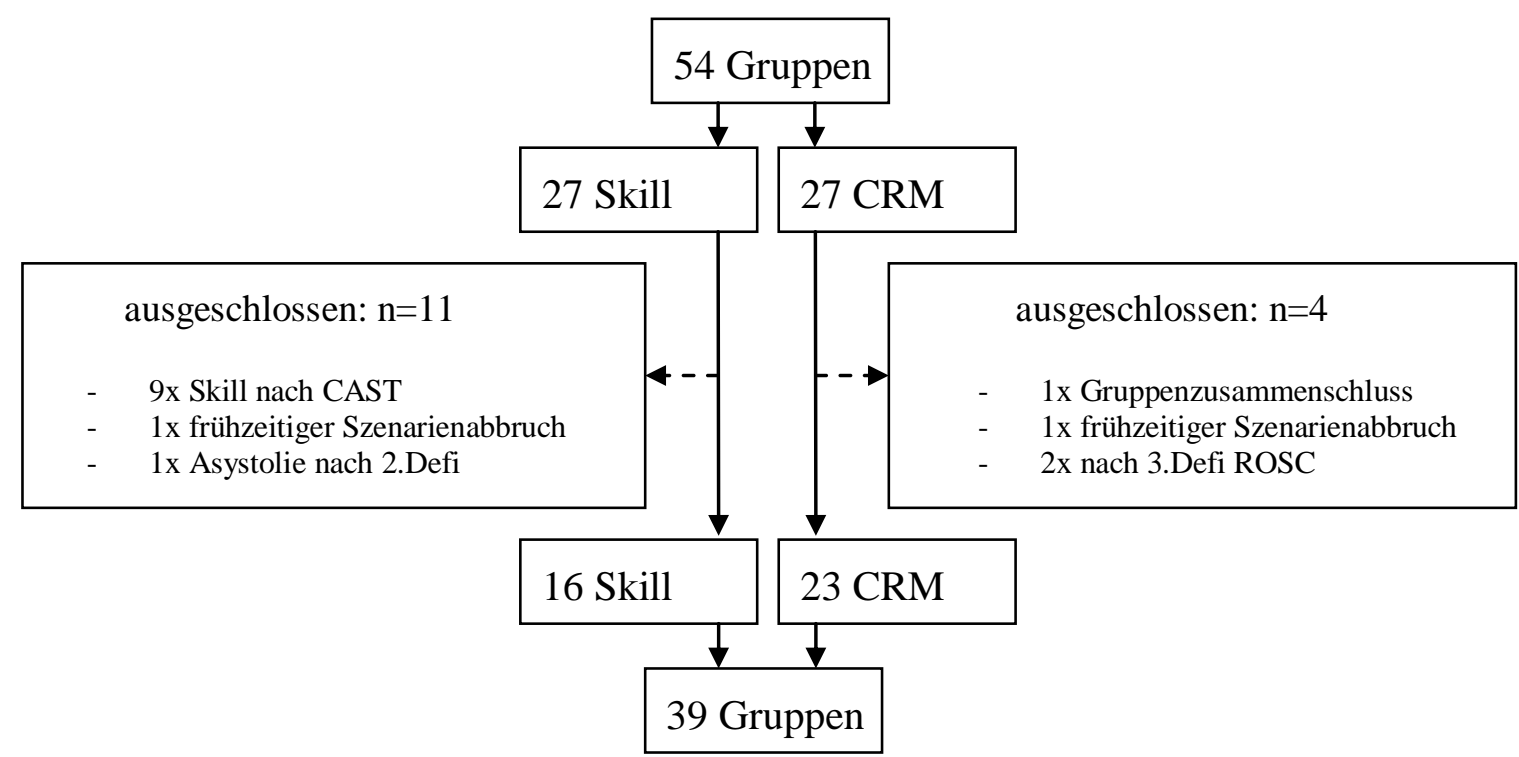

Abb. 3.1.a Flowchart des Probandenkollektivs in der Auswertung Video 


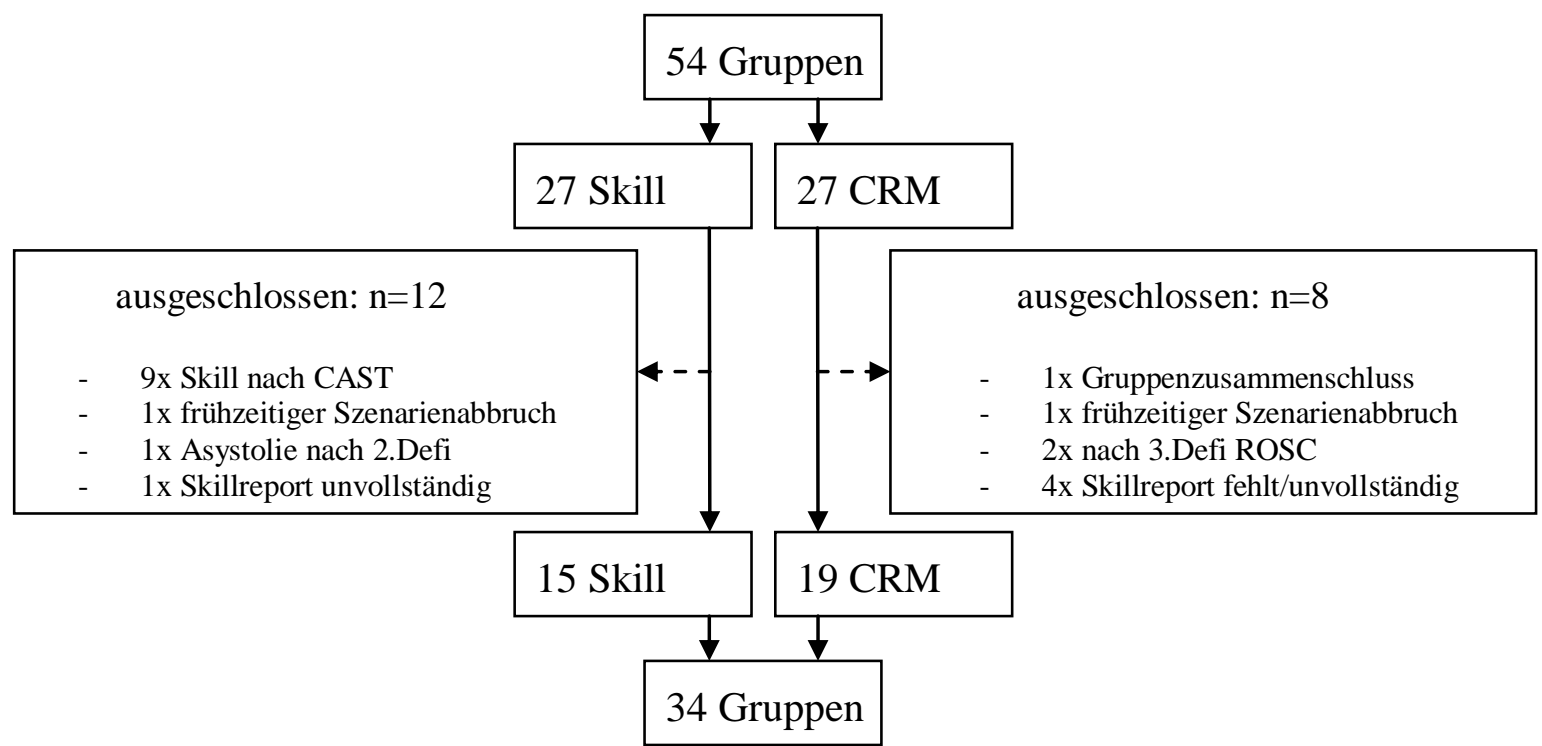

Abb. 3.1.b Flowchart des Probandenkollektivs in der Auswertung PC-Skillreport

Auch hinsichtlich der Geschlechterverteilung müssen die unterschiedlichen Gruppengrößen in den Auswertungsbereichen Algorithmuskonformität und Reanimationsqualität berücksichtigt werden. Die Geschlechterverteilung war in beiden Auswertungsbereichen nicht signifikant unterschiedlich.

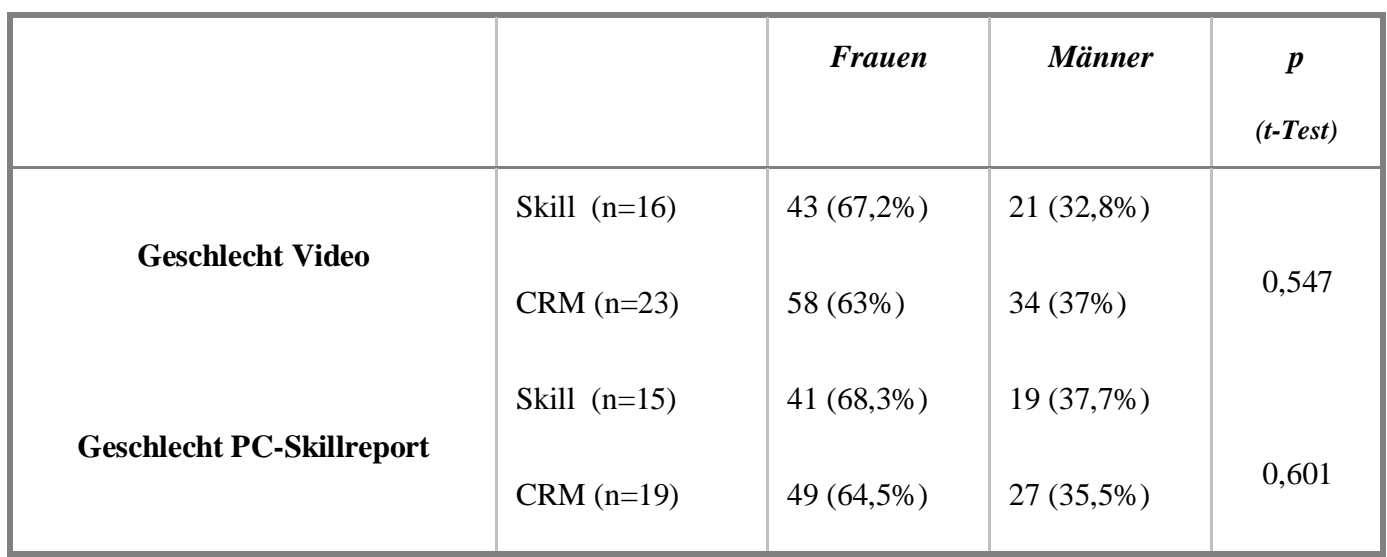

Tab 3.1 Geschlechterverteilung in den Kohorten 
Das mittlere Alter betrug in beiden Kohorten des Auswertungsbereiches Video 25,2 Jahre.

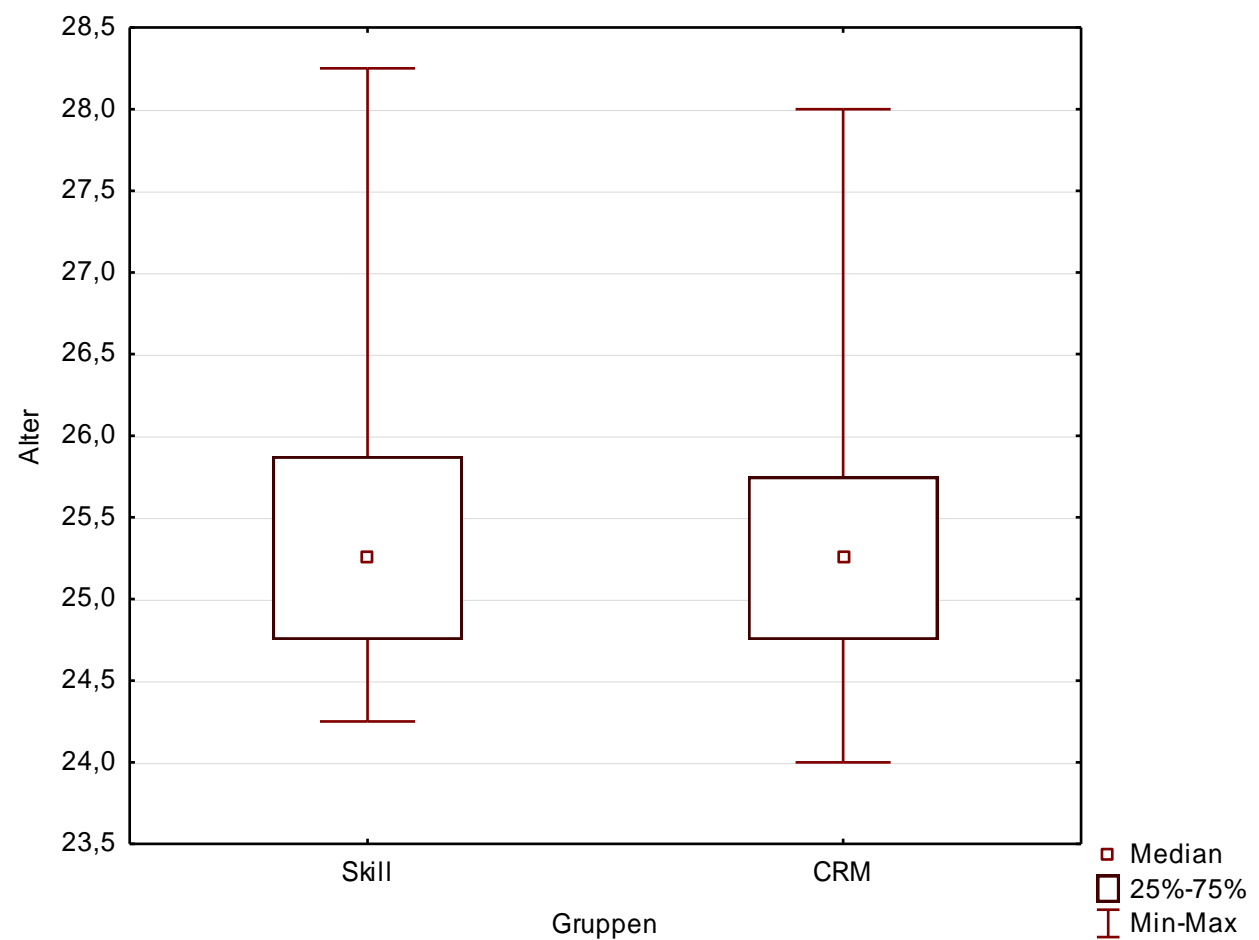

Abb. 3.1c Altersverteilung im Auswertungsbereich Video

Im Auswertungsbereich PC-Skillreport betrug das mittlere Alter der Skillgruppen 25,4 und der CRM-Gruppen 25,3 Jahre.

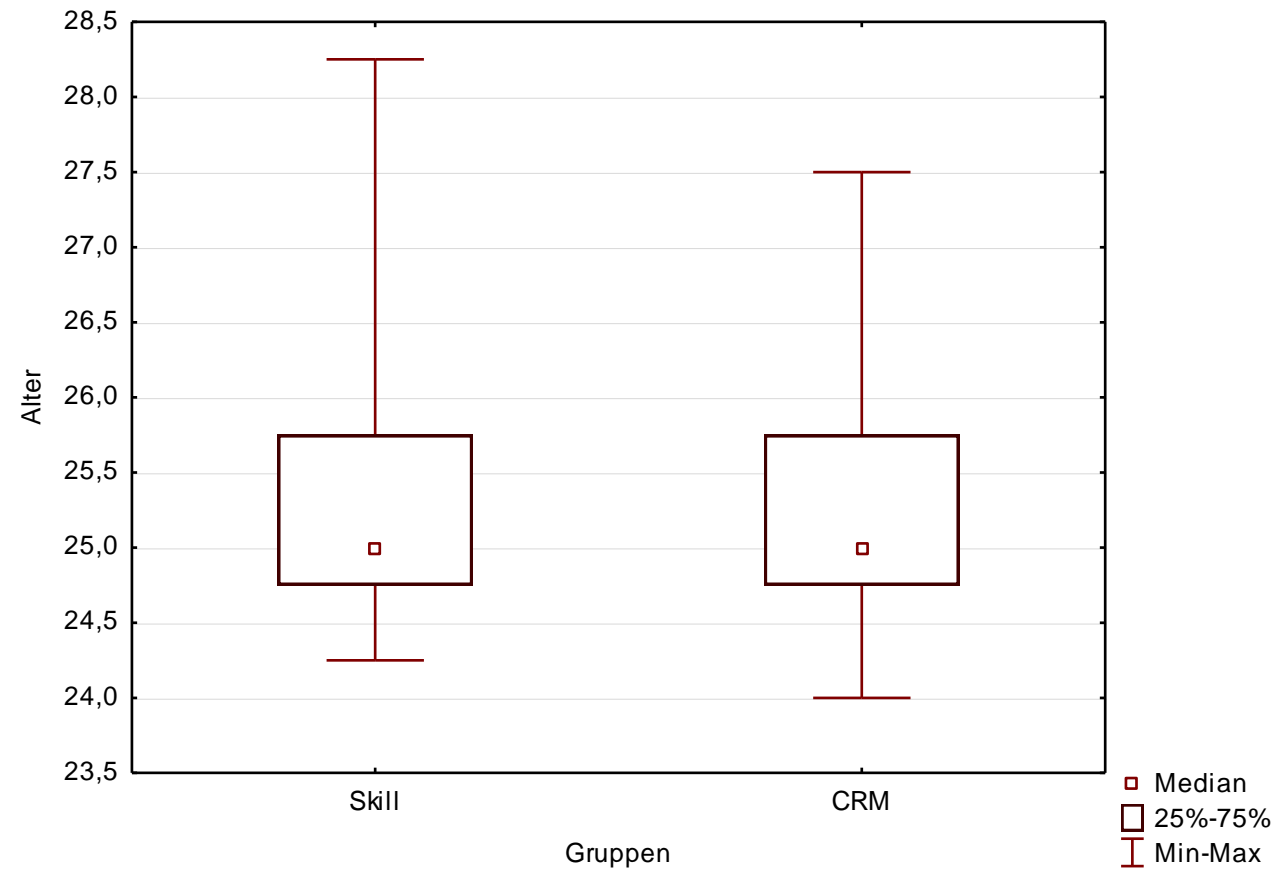

Abb. 3.1.d Altersverteilung im Auswertungsbereich PC-Skillreport 


\subsection{Auswertung Video}

Anhand des Videomaterials wurden mit Hilfe des Punktescores (Tab. 2.4.1) Vergleiche zwischen beiden Kohorten gezogen hinsichtlich der Parameter Basis-Check, Defibrillation, Medikamente/ i.v.-Zugang, Intubation/ asynchrone Beatmung, Rhythmuskonversion, ROSC und reversible Ursachen, die sich unter dem Begriff Algorithmuskonformität zusammenfassen lassen. Außerdem wurde mit den Videos die Zeit zur ersten adäquaten Intervention gemessen.

\subsubsection{Erste adäquate Intervention}

Hiermit ist der Zeitpunkt in Sekunden gemeint, zu dem die Gruppe nach Betreten des Raumes die erste Handlung durchführt, die therapeutische Zwecke verfolgt. Adäquat bedeutet im Fall des Kreislaufstillstandes Defibrillation, Beatmung oder Herzdruckmassage. Es ergaben sich keine signifikanten Unterschiede.

\begin{tabular}{|l|l|l|l|l|}
\hline & & Mittelwert & \multicolumn{1}{|c|}{$\boldsymbol{S D}$} & \multicolumn{1}{c|}{$\boldsymbol{p}$} \\
& & & & $(\boldsymbol{t}$-Test $)$ \\
\hline erste adäquate Intervention (sek) & Skill (n=16) & 28,5 & 19,22 & \\
& CRM (n=23) & 35,74 & 22,25 & 0,286 \\
\hline
\end{tabular}

Tab. 3.2.1a Erste adäquate Intervention

SD: Standardabweichung

\begin{tabular}{|l|c|c|c|}
\hline & Skill (n=16) & CRM (n= 23) & $\boldsymbol{p}$ \\
Herzdruckmassage & $14(87,5 \%)$ & $15(65,2 \%)$ & 0,2322 \\
Defibrillation & $1(6,25 \%)$ & $6(26,1 \%)$ & 0,2445 \\
Beatmung & $1(6,25 \%)$ & $2(8,7 \%)$ & 0,7422 \\
\hline
\end{tabular}

Tab. 3.2.1b Verteilung der Interventionsarten 


\subsubsection{Algorithmuskonformität}

Die Fallgruppen $(n=23)$ erreichten im Median 37 Punkte (minimal 24, maximal 57 Punkte), was einen signifikanten Unterschied $(p=0,005)$ zu den Kontrollgruppen $(n=16)$ ausmachte, die im Median nur 29 Punkte (minimal 15, maximal 42 Punkte) erreichten. Die maximal erreichbare Punktzahl waren 60 Punkte, ermittelt anhand des Punktescores (Tab. 2.4.1). Die Unterschiede zwischen Skill- und CRM-Gruppen bezüglich der einzelnen Parametern der Algorithmuskonformität werden im Folgenden erläutert.

\begin{tabular}{|l|c|c|c|c|}
\hline & & Median & Min-Max & $p$ \\
& & & & (MWU-Test $)$ \\
\hline Algorithmuskonformität & Skill (n=16) & 29 & $15-42$ & \\
& CRM (n=23) & 37 & $24-57$ & 0,005 \\
\hline
\end{tabular}

Tab. 3.2.2a Algorithmuskonformität

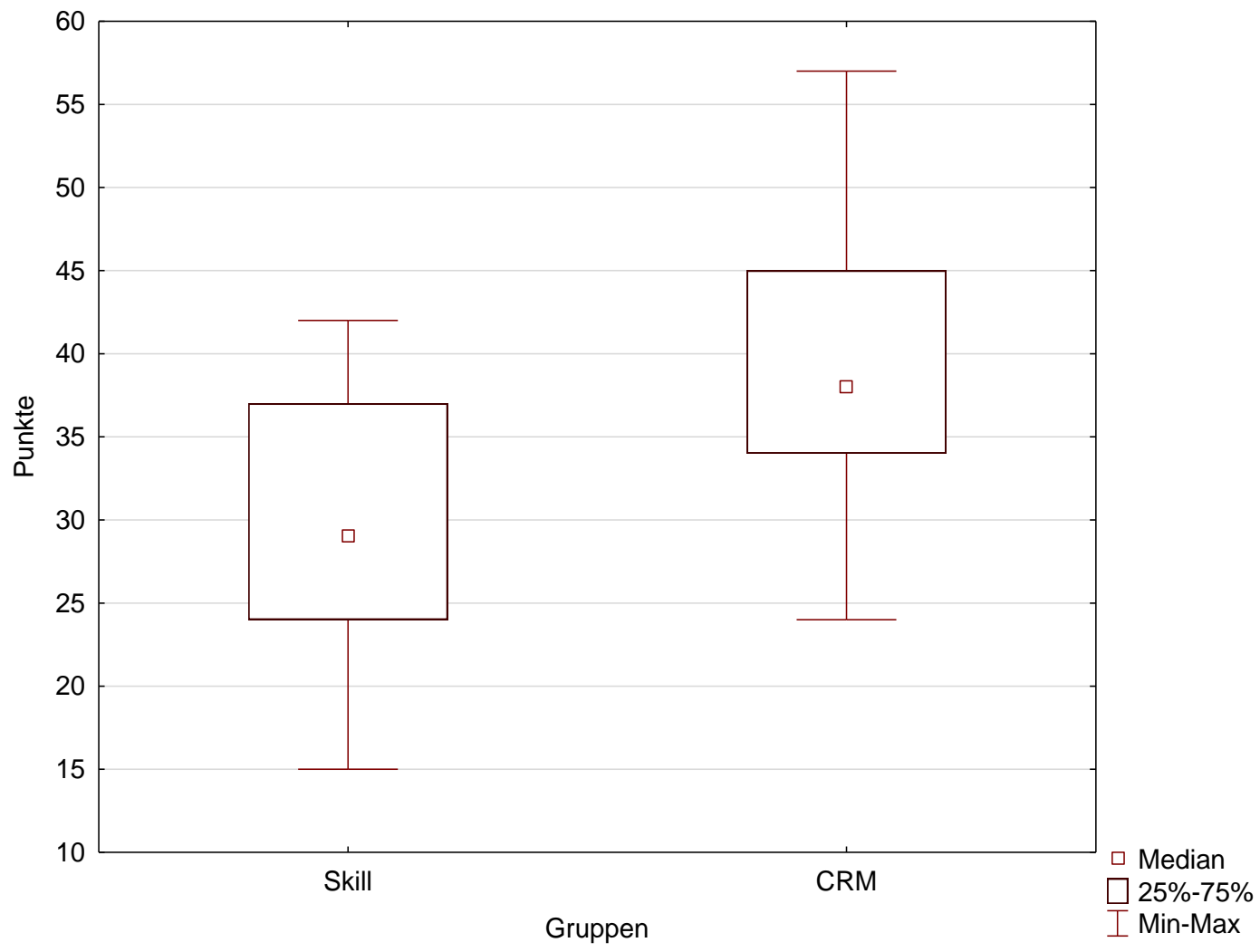

Abb. 3.2.2 Algorithmuskonformität 


\begin{tabular}{|c|c|c|c|c|c|}
\hline \multirow{2}{*}{ Kategorie } & \multirow[t]{2}{*}{ Unterkategorie } & \multicolumn{2}{|c|}{ Skill } & \multicolumn{2}{|c|}{$C R M$} \\
\hline & & Unterkat. & Kategorie & Unterkat. & Kategorie \\
\hline \multirow[t]{4}{*}{ Basis-Check } & Ansprechen & 1 & 3 & 1 & 2 \\
\hline & Atmung & 1 & & 1 & \\
\hline & Pulskontrolle & 0,5 & & 0 & \\
\hline & Diagnose Herz-Kreislaufstillstand & 0 & & 0 & \\
\hline \multirow[t]{3}{*}{ 1. Defi } & indizierte Durchführung & 1 & 2 & 1 & 2 \\
\hline & sicher ${ }^{1}$ und mit Gel durchgeführt & 1 & & 1 & \\
\hline & als Frühdefibrillation ${ }^{2}$ & 0 & & 0 & \\
\hline \multirow{4}{*}{ 2. Defi } & indizierte Durchführung & 1 & 3 & 1 & 3 \\
\hline & Zeitintervall HDM 2min +/- 30sek & 0 & & 0 & \\
\hline & $+/-15 \mathrm{sek}$ & 1 & & 0 & \\
\hline & sicher & 1 & & 1 & \\
\hline \multirow[t]{4}{*}{ 3. Defi } & indizierte Durchführung & 1 & 3,5 & 1 & 3 \\
\hline & Zeitintervall HDM 2min +/- 30sek & 0 & & 0 & \\
\hline & $+/-15 \mathrm{sek}$ & 0 & & 0 & \\
\hline & sicher & 1 & & 1 & \\
\hline \multirow[t]{4}{*}{ 4. Defi } & indizierte Durchführung & 1 & 3 & 1 & 3 \\
\hline & Zeitintervall HDM 2min +/- 30sek & 0 & & 0 & \\
\hline & $+/-15 \mathrm{sek}$ & 0 & & 0 & \\
\hline & sicher & 1 & & 1 & \\
\hline i.v. Zugang & ja & 2 & 2 & 2 & 2 \\
\hline \multirow[t]{2}{*}{ Adrenalin } & gegeben & 2 & 4 & 2 & 4 \\
\hline & zwischen 2. und 3.Schock & 2 & & 2 & \\
\hline \multirow[t]{2}{*}{ Amiodaron } & gegeben & 2 & 2 & 2 & 4 \\
\hline & zwischen 3. und 4.Schock & 0 & & 2 & \\
\hline \multirow[t]{2}{*}{ Intubation } & versucht & 2 & 2 & 2 & 4 \\
\hline & Lagekontrolle $^{3}$ & 2 & & 2 & \\
\hline HDM/Beatmung & kontinuierliche HDM nach Intubation & 0 & $\mathbf{0}$ & 5 & 5 \\
\hline \multirow[t]{4}{*}{ Rhythmuskonversion } & erkannt & 2 & -2 & 2 & -2 \\
\hline & weiter HDM & 4 & & 4 & \\
\hline & weiter HDM für $2 \mathrm{~min}$ & 0 & & 0 & \\
\hline & Abbruch HDM nach RK & -8 & & -8 & \\
\hline \multirow[t]{3}{*}{ ROSC } & Pulskontrolle zentral & 0 & 2 & 2 & 2 \\
\hline & RR-Kontrolle & 0 & & 0 & \\
\hline & post-resuscitation-care diskutiert & 0 & & 0 & \\
\hline reversible Ursachen & diskutiert & 0 & $\mathbf{0}$ & 0 & $\mathbf{0}$ \\
\hline GESAMT & & 29 & 29 & 37 & 37 \\
\hline
\end{tabular}

Tab. 3.2.2b Punktzahlen (Median) beider Kohorten in allen Kategorien der Algorithmuskonformität Unterkat.=Unterkategorie 


\subsubsection{Basis-Check}

In der Kategorie Basis-Check konnten maximal vier Punkte erreicht werden. Statistisch signifikante Unterschiede ergaben sich hierbei nicht. Die Skillgruppen erreichten im Median drei Punkte und die CRM-Gruppen zwei Punkte.

\begin{tabular}{|l|l|c|c|c|c|c|}
\hline & & Mittelwert & SD & Median & Min/Max & $p$ \\
& & & & & \\
\hline & Skill (n=16) & 2,63 & 0,62 & 3 & $2 / 4$ & \\
\hline \multirow{2}{*}{ Basis-Check } & CRM (n=23) & 2,35 & 0,49 & 2 & $2 / 3$ & 0,157 \\
& & & & & & \\
\hline
\end{tabular}

Tab. 3.2.2.1a Gesamtpunktzahl im Basis-Check

SD: Standardabweichung

Auch in den Unterkategorien bestanden keine signifikanten Unterschiede. Alle Gruppen sprachen während des Basis-Checks den Patienten an, mit gleichzeitiger Setzung von taktilen Reizen und überprüften die Atmung. Den Puls kontrollierten nur 50\% (Skill) bzw. 30,4\% (CRM) der Gruppen. Die Verbalisierung der Diagnose Herzkreislaufstillstand fand in den Skillgruppen zu 12,5\% und in den CRM-Gruppen zu 4,3\% statt.

\begin{tabular}{|l|c|c|c|}
\hline & Skill (n=16) & CRM (n=23) & $p$ \\
\hline Ansprache & $16(100 \%)$ & $23(100 \%)$ & - \\
Atemkontrolle & $16(100 \%)$ & $23(100 \%)$ & - \\
Pulskontrolle & $8(50 \%)$ & $7(30,4 \%)$ & 0,217 \\
Diagnose Herzkreislaufstillstand & $2(12,5 \%)$ & $1(4,3 \%)$ & 0,347 \\
\hline
\end{tabular}

Tab.3.2.2.1b Unterkategorien des Basis-Checks

Anzahl der Gruppen, die die einzelnen Bausteine des Basis-Checks ausführten (prozentual von n) 


\subsubsection{Defibrillation}

Für die vier Defibrillationen während des Szenarios wurden insgesamt maximal 20 Punkte vergeben. Somit ergaben sich für die indizierte Durchführung (defibrillierbarer Rhythmus: in diesem Fall immer Kammerflimmern) je 1 Punkt, maximal also 4 Punkte in dieser Unterkategorie, da pro Szenario maximal vier Defibrillationen indiziert waren. Punkte für das Einhalten des Zeitintervalls zwischen den Defibrillationen wurden vergeben wie in Kapitel 2.4.1 beschrieben. Maximal konnten dafür 6 Punkte erreicht werden. Für jedes sichere Defibrillieren gab es 1 Punkt, maximal also vier. Außerdem bekamen Gruppen, deren erste Defibrillation als Frühdefibrillation galt, 6 Punkte. Gruppen, die erst 90 Sekunden nach Szenarienbeginn zum ersten Mal defibrillierten, bekamen keinen Punkt. Unter den Skillgruppen wurden zwei Frühdefibrillationen durchgeführt, unter den CRMGruppen sieben. Statistisch signifikante Unterschiede bestanden nicht.

\begin{tabular}{|l|c|c|c|}
\hline & Skill (n=16) & CRM (n=23) & $\boldsymbol{p}$ \\
& & & $\left(\right.$ chi $\left.^{2}\right)$ \\
\hline Frühdefibrillation & $2(12,5 \%)$ & $7(30,4 \%)$ & 0,201 \\
\hline
\end{tabular}

Tab. 3.2.2.2a

Frühdefibrillation

\begin{tabular}{|l|l|l|l|l|l|l|}
\hline & Gruppen & Mittelwert & SD & Median & Min/ & \multicolumn{1}{|c|}{ Max } \\
(t-Test)
\end{tabular}

Tab. 3.2.2.2b Unterkategorien der Defibrillation und Gesamtpunktzahl Defibrillation SD: Standardabweichung 


\subsubsection{Medikamente/ i.v.-Zugang}

Medikamente, die in der Beurteilung der Reanimation eine Rolle spielten, waren Adrenalin und Amiodaron. Für die Gabe von Adrenalin/Amiodaron wurden 2 Punkte vergeben, abermals 2 Punkte gab es für den leitliniengerechten Zeitpunkt der Gabe, bei Adrenalin also zwischen der 2. und 3. Defibrillation. Amiodaron hingegen sollte zwischen dem 3.und 4. Schock verabreicht werden. Maximal 8 Punkte konnten in dieser Kategorie erreicht werden. Insgesamt erhielten die Skillgruppen im Median 5 Punkte und die CRM-Gruppen 6 Punkte, kein signifikanter Unterschied.

\begin{tabular}{|l|c|c|c|c|c|c|}
\hline & & Mittelwert & SD & Median & Min-Max & $\boldsymbol{p}$ \\
& & & & & & $($ MWU-Test $)$ \\
\hline & Skill (n=16) & 5,25 & 2,62 & 5 & $0-8$ & \\
\hline \multirow{2}{*}{ Medikamente } & CRM (n=23) & 6,61 & 1,53 & 6 & $4-8$ & 0,112 \\
& & & & & \\
\hline
\end{tabular}

Tab. 3.2.2.3a Gesamtpunktzahl Medikamente

SD: Standardabweichung

Jedoch unterschieden sich die Gruppen signifikant voneinander hinsichtlich der Unterkategorie „Amiodaron gegeben“. Alle CRM-Gruppen (100\%) gaben Amiodaron, wohingegen nur 62,5\% der Skillgruppen überhaupt Amiodaron verabreichten $(p<0,01)$.

\begin{tabular}{|c|c|c|c|}
\hline & Skill $(n=16)$ & $C R M(n=23)$ & $\begin{array}{c}p \\
\left(c h i^{2}\right)\end{array}$ \\
\hline Adrenalin gegeben & $15(93,8 \%)$ & $23(100 \%)$ & 0,225 \\
\hline Adrenalin zwischen 2./3. Defibrillation gegeben & $10(62,5 \%)$ & $17(73,9 \%)$ & 0,447 \\
\hline Amiodaron gegeben & $10(62,5 \%)$ & $23(100 \%)$ & 0,001 \\
\hline Amiodaron zwischen 3./4. Defibrillation gegeben & $7(43,8 \%)$ & $16(69,6 \%)$ & 0,107 \\
\hline
\end{tabular}

Tab. 3.2.2.3b Unterkategorien Medikamente

Gruppen, die die jeweilige Anforderung erfüllten, angegeben in absoluten Zahlen und Prozent 


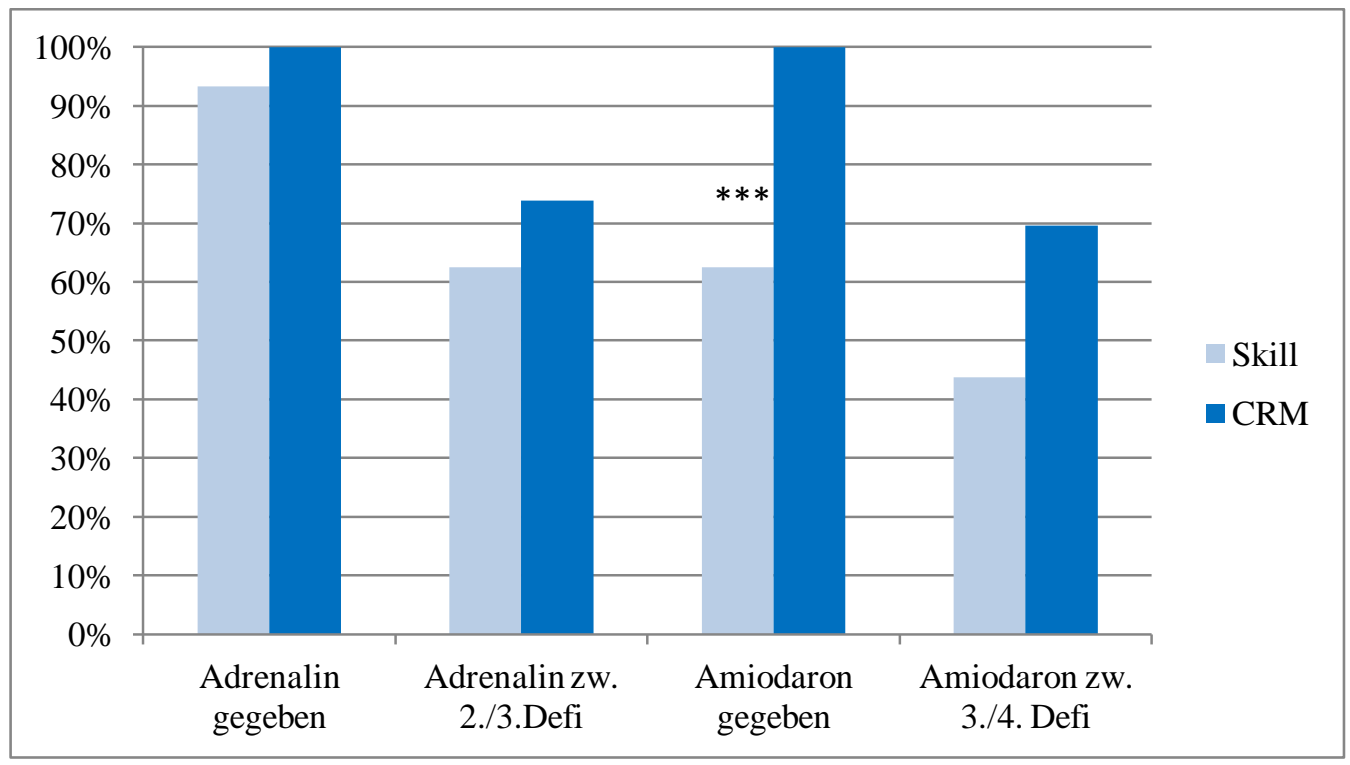

Abb. 3.2.2.3 Unterkategorien Medikamente $* * *: \mathrm{p}<0,001$

Alle Gruppen legten während der Reanimation einen intravenösen Zugang, über den die Medikamente verabreicht wurden. 


\subsubsection{Intubation/ asynchrone Beatmung}

Wenn eine Gruppe während der Reanimation versuchte zu intubieren, egal mit welchem Erfolg, wurden dafür 2 Punkte vergeben. Für die durchgeführte Lagekontrolle des Tubus nach Intubation gab es auch 2 Punkte. Von maximal 4 zu erreichenden Punkten brachten es die Skillgruppen im Median auf 2 Punkte und die CRM-Gruppen auf 4 Punkte, ein signifikantes Ergebnis $\mathrm{p}<0,001$.

\begin{tabular}{|l|c|c|c|c|c|c|}
\hline & & Mittelwert & SD & Median & Min/Max & $p$ \\
& & & & & & (MWU-Test $)$ \\
\hline \multirow{2}{*}{ Intubation } & Skill (n=16) & 1,88 & 1,86 & 2 & $0 / 4$ & \\
& CRM (n=23) & 3,91 & 0,42 & 4 & $2 / 4$ & $<0,001$ \\
\hline
\end{tabular}

Tab. 3.2.2.4a Intubation Gesamtpunktzahl SD: Standardabweichung

Auch die asynchrone Beatmung, die nach erfolgreicher endotrachealer Intubation indiziert war, führten im Mittel die CRM-Gruppen mit 82,6\% signifikant häufiger durch als die Skillgruppen mit 43,8\% ( $\mathrm{p}=0,017)$.

\begin{tabular}{|l|l|l|l|}
\hline & Skill (n=16) & CRM (n=23) & \multicolumn{1}{c|}{$\begin{array}{c}\boldsymbol{(} \\
\left(\text { chi }^{2}\right)\end{array}$} \\
\hline Intubation versucht & $9(56,3 \%)$ & $23(100 \%)$ & $<0,001$ \\
Lagekontrolle des Tubus durchgeführt & $6(37,5 \%)$ & $22(95,7 \%)$ & $<0,001$ \\
\hline asynchrone Beatmung nach Intubation & $7(43.8 \%)$ & $19(82,6 \%)$ & 0,017 \\
\hline
\end{tabular}

Tab. 3.2.2.4b Unterkategorien Intubation

Gruppen, die die jeweilige Anforderung erfüllten, angegeben in absoluten Zahlen und Prozent 


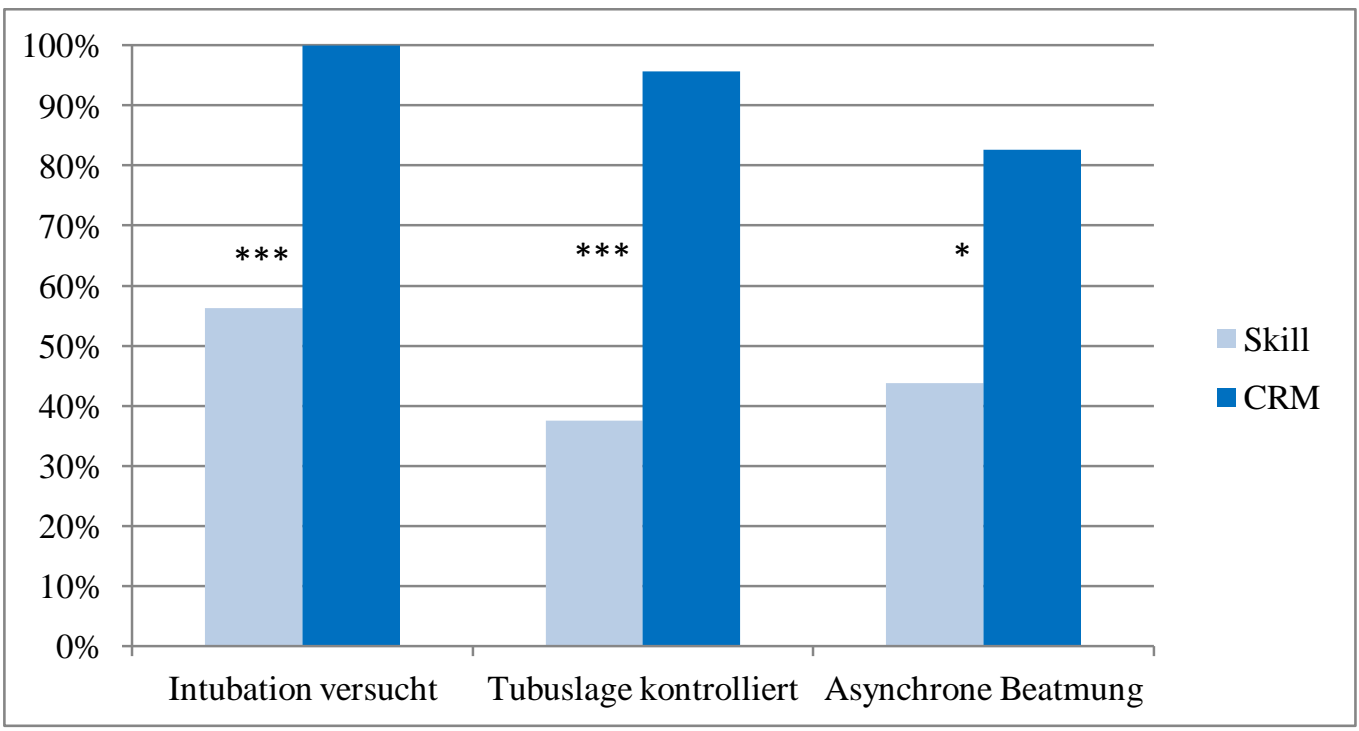

Abb. 3.2.2.4 Unterkategorien Intubation

$*$ : $\mathrm{p} \leq 0,05 \quad * * *: \mathrm{p} \leq 0,001$ 


\subsubsection{Rhythmuskonversion}

Die Kategorie Rhythmuskonversion lässt sich aufteilen in die Unterkategorien Rhythmuskonversion erkannt (2 Punkte), HDM fortgeführt (4 Punkte), HDM für 2 min fortgeführt (4 Punkte) und das Unterlassen der HDM nach Erkennen eines kreislaufeffektiven Rhythmus direkt nach der 4. Defibrillation. Letzteres hatte einen Abzug von 8 Punkten zur Folge, warum 10 Punkte die maximal und -4 Punkte die minimal erreichbare Punktzahl darstellten. Über Punktabzüge durch das oben erwähnte Unterlassen der Herzdruckmassage kamen die medianen Werte der Skill- und CRM-Gruppen von jeweils -2 Punkten zustande.

\begin{tabular}{|c|c|c|c|c|c|c|}
\hline & & Mittelwert & $S D$ & Median & $\operatorname{Min} / \operatorname{Max}$ & $\begin{array}{c}p \\
(M W U-T e s t)\end{array}$ \\
\hline Rhythmuskonversion & $\begin{array}{l}\text { Skill }(n=16) \\
\text { CRM }(n=23)\end{array}$ & $\begin{array}{l}2,38 \\
3,48\end{array}$ & $\begin{array}{l}5,85 \\
6,07\end{array}$ & $\begin{array}{l}-2 \\
-2\end{array}$ & $\begin{array}{l}-2 / 10 \\
-4 / 10\end{array}$ & 0,678 \\
\hline
\end{tabular}

Tab. 3.2.2.5a Gesamtpunktzahl Rhythmuskonversion

SD: Standardabweichung

\begin{tabular}{|c|c|c|c|}
\hline & Skill $(n=16)$ & $C R M(n=23)$ & $\begin{array}{c}p \\
\left(c h i^{2}\right)\end{array}$ \\
\hline Rhythmuskonversion erkannt & $15(93,8 \%)$ & $20(87 \%)$ & 0,492 \\
\hline HDM fortgeführt & $16(100 \%)$ & $23(100 \%)$ & - \\
\hline HDM für 2 min fortgeführt & $6(37,5 \%)$ & $11(47,8 \%)$ & 0,522 \\
\hline Abbruch der HDM nach Erkennen der Rhythmuskonversion & $9(56,3 \%)$ & $12(52,2 \%)$ & 0,802 \\
\hline
\end{tabular}

Tab. 3.2.2.5b Unterkategorien Rhythmuskonversion

Gruppen, die die jeweilige Anforderung erfüllten, angegeben in absoluten Zahlen und Prozent 


\subsubsection{ROSC}

Die Rückkehr eines Herzrhythmus, der mit ausreichender Kreislaufaktivität einhergehen kann (return of spontaneous circulation=ROSC) erfordert Kontrollen von Puls (Carotis), Blutdruck und die Planung des weiteren Vorgehens (post-resuscitation-care). Diese drei Aspekte wurden bei Erfüllen mit je 2 Punkten belohnt, hier also maximal 6 Punkte. Im Median erreichten beide Gruppen 2 Punkte.

\begin{tabular}{|c|c|c|c|c|c|c|}
\hline & & Mittelwert & $S D$ & Median & $\operatorname{Min} / \operatorname{Max}$ & $\begin{array}{c}p \\
(M W U-T e s t)\end{array}$ \\
\hline ROSC & $\begin{array}{l}\text { Skill }(n=16) \\
\text { CRM }(n=23)\end{array}$ & $\begin{array}{l}1,75 \\
2,78\end{array}$ & $\begin{array}{l}1,77 \\
1,98\end{array}$ & $\begin{array}{l}2 \\
2\end{array}$ & $\begin{array}{l}0 / 6 \\
0 / 6\end{array}$ & 0,097 \\
\hline
\end{tabular}

Tab. 3.2.2.6a Gesamtpunktzahl ROSC

SD: Standardabweichung

\begin{tabular}{|l|l|l|l|}
\hline & Skill (n=16) & CRM (n=23) & $p$ \\
& & & (chi' $^{2}$ \\
\hline Pulskontrolle zentral & $7(43,8 \%)$ & $17(73,9 \%)$ & 0,057 \\
Blutdruckkontrolle & $4(25 \%)$ & $8(34,8 \%)$ & 0,515 \\
post-resuscitation-care diskutiert & $3(18,8 \%)$ & $7(30,4 \%)$ & 0,411 \\
\hline
\end{tabular}

Tab. 3.2.2.6b Maßnahmen nach ROSC

Gruppen, die die jeweilige Anforderung erfüllten, angegeben in absoluten Zahlen und Prozent 


\subsubsection{Reversible Ursachen}

Wenn während der Reanimation mindestens eine von acht möglichen reversiblen Ursachen (siehe 2.4.1.7) für den Kreislaufstillstand in der Gruppe erwähnt bzw. diskutiert wurde, bekam die Gruppe dafür einen Punkt. Dies geschah in den Skillgruppen zu 31,3\% und in den CRM-Gruppen zu 47,8\%.

\begin{tabular}{|l|l|l|l|}
\hline & Skill (n=16) & CRM $(\boldsymbol{n = 2 3})$ & $\boldsymbol{p}$ \\
$\left(\boldsymbol{c h i}^{2}\right)$ \\
\hline reversible Ursachen diskutiert & $5(31,3 \%)$ & $11(47,8 \%)$ & 0,301 \\
\hline
\end{tabular}

Tab. 3.2.2.7 Reversible Ursachen

Gruppen, die die jeweilige Anforderung erfüllten, angegeben in absoluten Zahlen und Prozent 


\subsection{Auswertung Skillreport}

Aus den Aufzeichnungen des PC-Skillreporting Systems (C) wurden die Reanimationsskills Herzdruckmassage, Beatmung und NFT ermittelt. Unvorhersehbare technische Probleme führten zu Datenverlusten, sodass bei den Auswertungen für Skill nur 15 Gruppen und für CRM nur 19 Gruppen berücksichtigt werden konnten (Abb. 3.1b). Es wurden jeweils Vergleiche zwischen den Leistungen der CRM- und der Kontrollgruppen gezogen.

\subsubsection{NFT}

Die no-flow time (Zeit ohne Herzdruckmassage) wurde für jede Gruppe in Sekunden pro Minute ausgewertet, in Prozent ausgedrückt und dann gemittelt. Die CRM-Gruppen führten vom Zeitpunkt der ersten adäquaten Intervention bis zur vierten Defibrillation eine längere Zeit Herzdruckmassage aus als die Skillgruppen, das heißt ihre NFT war

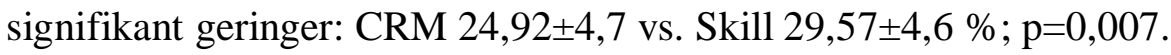

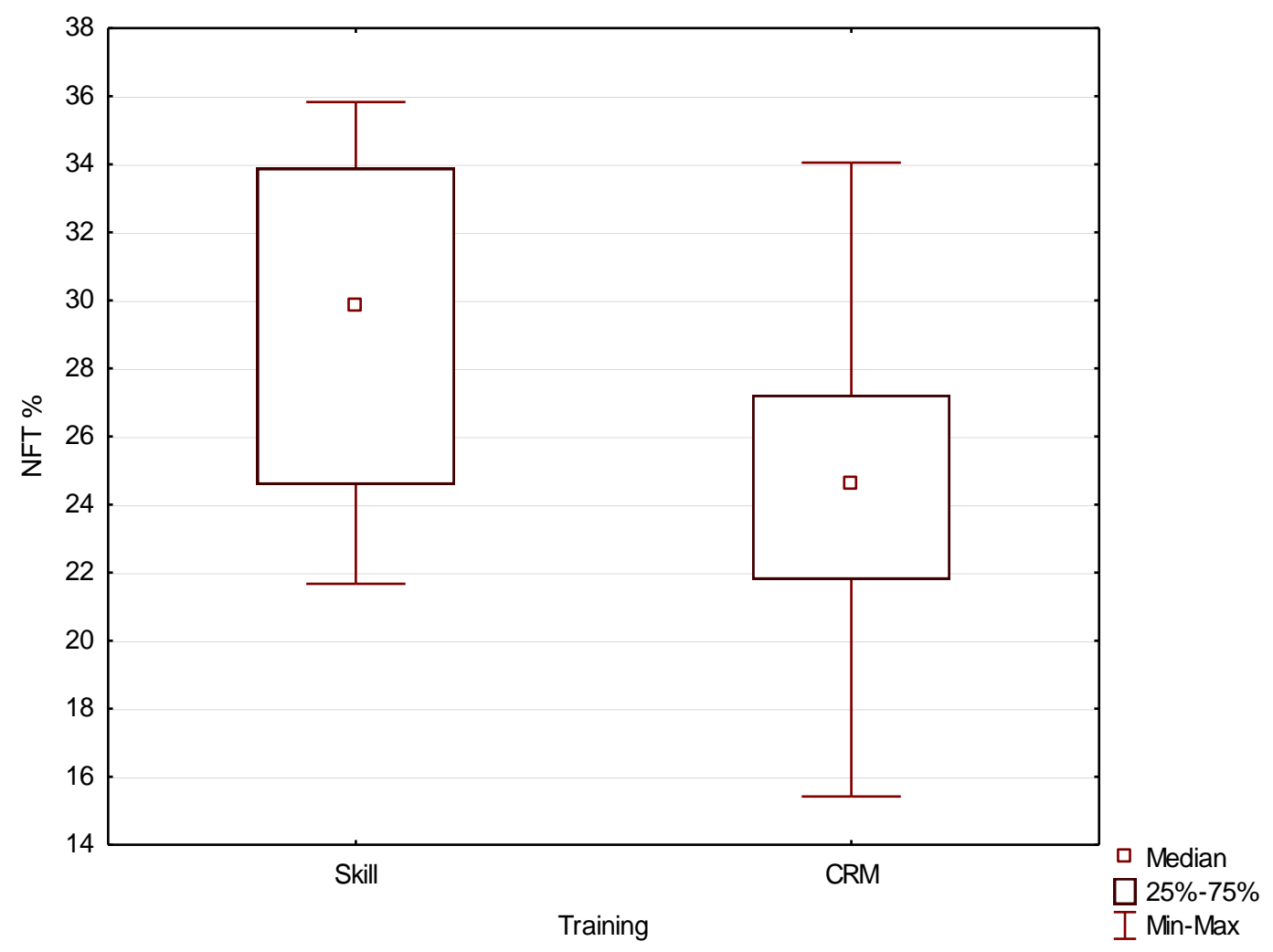

Abb. 3.3.1 no-flow time 


\subsubsection{Herzdruckmassage}

Hinsichtlich der vier zu untersuchenden Aspekte der Herzdruckmassage (Frequenz, Drucktiefe, Entlastung, Handposition) ergaben sich zwischen den beiden Gruppen keine signifikanten Unterschiede.

\begin{tabular}{|l|l|l|l|l|l|}
\hline & \multicolumn{2}{|c|}{ Skill (n=15) } & \multicolumn{2}{c|}{ CRM (n=19) } & $p$ \\
& Mittelwert & \multicolumn{1}{c|}{ SD } & Mittelwert & \multicolumn{1}{c|}{ SD } & $(t$-Test $)$ \\
\hline Druckfrequenz/min & 106 & 13,5 & 109 & 14,08 & 0,561 \\
Drucktiefe \% korrekt & 50,21 & 18,99 & 52,56 & 25,03 & 0,757 \\
Entlastung \% korrekt & 83,83 & 17,73 & 85,83 & 19,56 & 0,763 \\
Handposition \% korrekt & 76,47 & 22,32 & 81,36 & 29,94 & 0,589 \\
\hline
\end{tabular}

Tab. 3.3.2 Qualität und Quantität der Herzdruckmassage SD: Standardabweichung

\subsubsection{Maskenbeatmung}

Die Daten beschränkten sich lediglich auf die korrekten Maskenbeatmungen pro Minute anteilig von den gesamten Maskenbeatmungen pro Minute (Angabe in Prozent). In ihrer Beatmungskompetenz unterschieden sich die Gruppen statistisch nicht voneinander.

\begin{tabular}{|c|c|c|c|c|}
\hline & & Mittelwert & $S D$ & $\begin{array}{c}p \\
(t-T e s t)\end{array}$ \\
\hline $\begin{array}{c}\text { korrekte Maskenbeatmungen/min } \\
(\%)\end{array}$ & $\begin{array}{l}\text { Skill }(n=15) \\
\text { CRM }(n=19)\end{array}$ & $\begin{array}{l}61,09 \\
51,97\end{array}$ & $\begin{array}{l}28,67 \\
34,55\end{array}$ & 0,407 \\
\hline
\end{tabular}

Tab. 3.3.3 Maskenbeatmung

SD: Standardabweichung 


\section{DISKUSSION}

\subsection{Gesamtergebniss}

In der vorliegenden Arbeit wurde der Effekt eines CRM-Trainings als Teil des medizinischen Curriculums auf die Reanimationsqualität und Leitlinienadhärenz im simulierten ALS unter besonderer Beachtung der NFT und der Algorithmuskonformität untersucht.

In einigen der untersuchten Parameter traten statistisch signifikante Unterschiede in den Leistungen zwischen den CRM- und Skillgruppen auf. Die CRM-Gruppen schnitten in den Kategorien Algorithmuskonformität und NFT signifikant besser ab als die Skillgruppen. In einer gleichzeitig durchgeführten Untersuchung ergaben sich positive Effekte des CRMTrainings auch auf kommunikationspsychologischer Ebene: die CRM-Gruppen zeigten signifikant mehr Teamleiter-Verbalisationen in den Kategorien direkte/indirekte Aufforderungen, Aufgabenverteilung und Planung als die teamleitende Person der Skillgruppen. Diese Kategorien repräsentieren auch wünschenswertes CRM-Verhalten, das die Gruppen, die das vorherige CRM-Seminar besucht hatten, sehr stark zeigten (Fernandez Castelao et al. 2011).

\subsection{Video}

\subsubsection{Erste adäquate Intervention}

Da der frühzeitige Beginn der kardiopulmonalen Reanimation zu einem besseren Outcome führt (Wik et al. 1994), ist es interessant diesen Aspekt zu untersuchen. Wie den Tabellen 3.2.1 $\mathrm{a}$ und $\mathrm{b}$ zu entnehmen, konnte kein statistisch signifikanter Unterschied hinsichtlich des Zeitpunktes und der Art der ersten adäquaten Intervention der Gruppen ermittelt werden. Es zeigte sich aber eine Tendenz, dass die Skillgruppen früher intervenierten. Nach durchschnittlich 28,5 Sekunden begannen die Teams der Skillgruppen überwiegend mit Herzdruckmassage (87,5\%). Nur zu jeweils 6,25\% wurde defibrilliert oder beatmet. Die verspätete erste adäquate Intervention der CRM-Gruppen, 35,7 Sekunden nach Szenarienbeginn, könnte mit dem höheren Anteil von Defibrillationen als erste adäquate Intervention zusammenhängen. $\mathrm{Zu}$ 26,1\% wurde unter den CRM-Gruppen defibrilliert, was einen größeren zeitlichen Aufwand bedeutet, als Herzdruckmassage durchzuführen, da 
zunächst der Defibrillator eingeschaltet, Elektrodengel aufgetragen, eine Rhythmusanalyse durchgeführt und auf 360 Joule geladen werden muss. Die Defibrillation ist die wünschenswerte erste Intervention bzw. die einzig korrekte der drei Möglichkeiten bei beobachtetem Kreislaufstillstand mit Kammerflimmern (Wenzel et al. 2006). Auch Hunziker et al. nutzen den Parameter der ersten adäquaten Intervention zur Beurteilung von CPR und Teambildung. Jedoch unterscheidet sich das Studiendesign grundlegend, da die Probanden Ärzte waren und auch nicht mit CRM-Training gearbeitet wurde, sondern nur mit spontaner vs. früher Teambildung (Hunziker et al. 2009).

Fasst man alles zusammen, so lässt sich vermuten, dass durch die CRM-Schulung ein höherer, wenn auch nicht signifikanter Anteil an Defibrillationen als erste adäquate Intervention durchgeführt wurde und somit wegen dessen größeren zeitlichen Aufwands, von den CRM-Gruppen erst später interveniert wurde. Das bedeutet gleichzeitig, dass sich die CRM-Gruppen in der frühen Phase der Reanimation enger an den Algorithmus hielten, der eine sofortige Defibrillation bei beobachtetem Kreislaufstillstand erfordert (dazu auch Kapitel 4.2.2). Ein anderes Argument für die frühere Intervention der Skillgruppen in Form von Herzdruckmassage könnte sein, dass in der Aufregung des Szenarienbeginns aus Verlegenheit schnell mit Herzdruckmassage begonnen wird, da dies eine relativ einfache Aufgabe ist und sich somit die erste Defibrillation verzögert. Es könnte sein, dass die Studierenden sich eher davor scheuen, einen Defibrillator zu nutzen, wenn sie nicht dazu aufgefordert werden oder nicht fest für das Defibrillieren eingeteilt sind. Durch die hohe Aufgabenkomplexität in der Anfangsphase einer Reanimation ist der initiale Bedarf an Koordination und Teamleitung hoch (Fernandez Castelao et al. 2011). Dies könnte durch CRM-Training verbessert und dadurch wertvolle Zeit durch effektive Aufgabenverteilung gewonnen werden. Um weitere Gründe für das langsamere Intervenieren der CRMGruppen zu suchen, müsste untersucht werden, ob es vielleicht an der Unerfahrenheit der Studierenden im Umgang mit dem Defibrillator liegen könnte.

\subsubsection{Algorithmuskonformität}

Da sich das Outcome der Patienten mit der Einführung der Reanimationsleitlinien 2005 verbessert hat (Olasveengen et al. 2009), sollte es eine logische Konsequenz sein, sich im Falle einer kardiopulmonalen Reanimation eng an den Reanimationsalgorithmus zu halten. Jedoch konnte dies, wie Studien zeigen, bislang nur mangelhaft umgesetzt werden (Marsch 
et al. 2005a; Wik et al. 2005). Eine Methode, um diese Algorithmuskonformität zu steigern, könnte die Einführung der Lehre von Crisis Resource Management sein, wie die vorliegende Arbeit zeigt: Studierende, die vor der Datenerhebung ein CRM-Training besuchten, hielten sich signifikant enger an den ALS-Algorithmus, als die Gruppen, die lediglich die für die Reanimation benötigten „Skills“ wiederholten. Die CRM-Gruppen erreichten von 60 möglichen Punkten für die Algorithmuskonformität im Median 37 Punkte, während die Skillgruppen nur 29 Punkte erreichten. Diese Ergebnisse deuten darauf hin, dass die Schulung in Crisis Resource Management die Algorithmuskonformität während einer CPR erhöhen könnte. In der Literatur finden sich keine Beispiele zu eventuellen Zusammenhängen zwischen CRM-Training und ALS-Algorithmuskonformität. Die These wird aber auch von den Ergebnissen der kommunikationspsychologischen Untersuchung gestützt, denn die CRM-Gruppen wiesen einen signifikant größeren Anteil von Teamleiter-Verbalisationen auf als die Skillgruppen (Fernandez Castelao et al. 2011) und übertrugen somit ihr Wissen des CRM-Trainings auch in die Praxis. Mehr Teamleiter-Verbalisationen, die durch das vorherige CRMTraining begründet werden könnten, die Handlungen gemäß des ALS-Algorithmus fordern, würden somit eine höhere ALS-Algorithmuskonformität durch CRM-Training bedeuten. Es lässt sich vermuten, dass für die leitliniengerechte Reanimation das CRMTraining eine sehr wichtige Komponente ist und es seinen festen Platz in der ALSAusbildung von Medizinstudenten und Ärzten erhalten sollte.

Im Folgenden wird die Relevanz der einzelnen Parametern des Algorithmus erörtert und dessen Einfluss auf das Gesamtergebniss der Algorithmuskonformität beschrieben.

\subsubsection{Basis-Check}

Hinsichtlich des Basis-Checks gab es keine Unterschiede zwischen den Gruppen. Ein Zusammenhang wurde hierbei aber auch initial nicht vermutet, da die Studierenden schon im ersten klinischen Semester Unterricht im Basic Life Support erhielten, wie es der Lehrplan in Göttingen vorschreibt, wo die Komponente Basis-Check geübt und auch geprüft wurde. Es konnte hierbei also ein ungefähr gleiches Leistungslevel unter den Studierenden erwartet werden. 


\subsubsection{Defibrillation}

Die erste Defibrillation hatte unter den einzelnen Parametern der Algorithmuskonformität ein großes Gewicht. Sie wurde, falls innerhalb von 90 Sekunden nach Szenariobeginn durchgeführt, mit sechs Punkten bewertet, denn die Überlebenschance bei Kammerflimmern sinkt mit jeder Minute ohne Defibrillation um etwa 10\% (Weaver et al. 1988). Diese sogenannte Frühdefibrillation wurde von den CRM-Gruppen häufiger ausgeführt als von den Skillgruppen (30,4 vs. 12,5\%), jedoch konnte sich kein statistisch signifikanter Unterschied nachweisen lassen. Eine Erklärung hierfür könnte sein, dass die Studierenden noch nicht erfahren genug im Umgang mit dem Gerät waren, um schneller die erste Defibrillation durchzuführen und somit außerhalb der 90-Sekunden-Grenze agierten. Die ärztlichen Probanden der vorab formierten Gruppe bei Hunziker et al. gaben durchschnittlich innerhalb von 67 Sekunden nach Eintritt von Kammerflimmern den ersten Schock ab (Hunziker et al. 2009), was nach der 90-Sekunden-Definition als Frühdefibrillation gilt. Ärzte defibrillieren im Vergleich zu Studierende mehrheitlich früher, was unterstreichen könnte, dass mehr Erfahrung und Übung zur schnelleren Defibrillation führt.

Die weiteren Ergebnisse der Unterkategorien im Abschnitt Defibrillation können der Tabelle 3.2.2.2b entnommen werden, es konnten keine weiteren Unterschiede aufgezeigt werden.

\subsubsection{Medikamente}

Bei den erforderlichen Medikamenten im untersuchten Szenario handelt es sich um Adrenalin und Amiodaron. In der Gabe und dessen Zeitpunkt von Adrenalin fanden sich keine Unterschiede. Amiodaron hingegen wurde von den CRM-Gruppen signifikant häufiger gegeben als von den Skillgruppen (100\% vs. 62,5\%), jedoch ohne Unterschied hinsichtlich dem Zeitpunkt der Gabe. Eine mögliche Erklärung hierfür ist, dass Adrenalin ein sehr bekanntes Medikament in der Notfallmedizin ist und vermutlich bei vielen Studierenden mit der Therapie eines Kreislaufstillstandes in Verbindung gebracht wird. Amiodaron dagegen ist ein Medikament aus der komplexen Klasse der Antiarrhythmika aus der Kardiologie bzw. Notfallmedizin und eventuell den Studierenden nicht geläufig genug, um in einer Stresssituation an Amiodaron und seine korrekte Dosierung zu denken. Genau in dieser Stresssituation hat ein unabhängiger Teamleiter mehr Zeit, über die 
nächsten Schritte des Algorithmus nachzudenken und diese als Anweisungen weiterzuleiten, eben wie die Gabe von Amiodaron.

\subsubsection{Intubation/ asynchrone Beatmung}

Ein sehr eindeutiges und auch signifikantes Ergebniss war, dass die CRM-Gruppen im Median 4 von 4 Punkten für die Kategorie Intubation bekamen (Intubation versucht und Lagekontrolle=jeweils zwei Punkte), wohingegen von den Skillgruppen im Median nur 2 Punkte erreicht wurden. Durch die Intubation bestand dann bei den erfolgreichen Gruppen auch die Möglichkeit zur asynchronen Beatmung (Beatmung unter laufender Herzdruckmassage), was durch die Ergebnisse hinsichtlich der versuchten Intubation (Skill $56,3 \%$ vs. CRM 100\%) auch signifikant häufiger von CRM-Gruppen geleistet wurde als von Skillgruppen (82,6\% vs. 43,8\%). Für Studierende ist die Intubation eine sehr anspruchsvolle Aufgabe, da sie viel Übung und klinische Erfahrung erfordert bis sie zur Routine wird. Man kann sich deshalb vorstellen, dass eine gewisse Hemmschwelle besteht unter Stress, Zeitdruck, Beobachtung und suboptimalen Bedingungen (Puppe liegt auf dem Boden) einen Intubationsversuch zu wagen. Diese Hemmschwelle wurde vielleicht bei den CRM-Gruppen herabgesetzt, da die Rollen klar verteilt waren, sich der/die Zuständige/r für das Airwaymanagement verantwortlich fühlte und somit versuchte zu Intubieren. Auch wurde die Intubation wahrscheinlich durch den Teamleiter klar gefordert und organisiert, sodass der Zuständige keine andere Wahl hatte als es $\mathrm{zu}$ versuchen. Es wurde nicht bewertet, ob die Intubation erfolgreich war oder nicht, da dies nicht prüfungsrelevant war und von Studierenden mit wenig klinischer Erfahrung nicht erwartet werden kann. Für das schlechte Abschneiden der Skillgruppen könnte wieder die mangelnde Teamführung eine Erklärung sein. So wurden die Studierenden von niemandem aufgefordert zu intubieren und hielten sich vermehrt an die Maskenbeatmung.

Da die asynchrone Beatmung nur nach erfolgreicher Intubation erfolgen kann, machten die Skillgruppen auch Verluste in der Gesamtpunktzahl, denn die asynchrone Beatmung wurde mit fünf Punkten bewertet. Begründet wird die verhältnismäßig hohe Punktzahl durch die wichtige Reduzierung der NFT aufgrund der asynchronen Beatmung, die eine durchgängige Herzdruckmassage ermöglicht. Im Falle einer unerkannten Fehlintubation, erkennbar aus den Aufzeichnungen des PC-Skillreports und nachfolgender fälschlicher asynchroner Beatmung, wurden trotzdem fünf Punkte vergeben, da es vermieden werden 
sollte den tatsächlichen Intubationserfolg bzw. Misserfolg zu bewerten. Auch in der Literatur sind Empfehlungen für die Einführung von CRM in die Ausbildung des Airwaymanagements zu finden (Rall und Dieckmann 2005), jedoch konnte noch kein Zusammenhang zwischen CRM und Airwaymanagement im ALS nachgewiesen werden.

In der Literatur wird vermehrt der Einsatz von Atemwegsalternativen bei CPR bzw. für den unerfahrenen Anwender diskutiert. Studierende ohne Erfahrung im Airwaymanagement intubierten am Patienten schneller und erfolgreicher mit Intubationslarynxmasken als über die endotracheale Intubation (Timmermann et al. 2007), jedoch wurde diese Studie am Patienten im OP durchgeführt, wo eine höhere Patientensicherheit gewährleistet werden konnte als in einer Situation mit CPR. Reiter et al. zeigten, dass der Gebrauch von Larynxmasken bei CPR die Zeit bis zur Sicherung der Atemwege signifikant verkürzt (Reiter et al. 2013). Auch der Einsatz von Larynxmasken statt endotrachealer Tuben bei CPR von Kindern führt zur schnelleren Atemwegssicherung, effektiveren Ventilation und geringerer Komplikationsrate (Chen und Hsiao 2008).

In der vorliegenden Arbeit wurden den Studierenden im Notfallkoffer neben endotrachealen Tuben auch Larynxmasken zur Verfügung gestellt, jedoch wurden sie in keinem Fall verwendet. Vielleicht müsste vermehrt auf die mögliche Nutzung von Atemwegsalternativen während CPR in den Lehrveranstaltungen hingewiesen werden, um die Intubationsbereitschaft bei den Studierenden zu erhöhen, die sich vor endotrachealer Intubation scheuen.

Es bleibt zu untersuchen, ob die Nutzung von Atemwegsalternativen während einer simulierten CPR eine niedrigere Hemmschwelle für Studierende bedeutet, die im Umgang mit der endotrachealen Intubation unerfahren sind und ob dies $\mathrm{zu}$ einer höheren Intubationsbereitschaft führen könnte.

\subsubsection{Rhythmuskonversion, ROSC und reversible Ursachen}

In den Kategorien Rhythmuskonversion, ROSC, und reversible Ursachen gab es keine signifikanten Unterschiede zwischen den Gruppen, jedoch gibt es diskussionswürdige Hinweise bezüglich des Verhaltens der Studierenden.

Nach der vierten Defibrillation, die in dem gewählten Szenario immer die Konversion in einen Sinusrhythmus zu Folge hatte, setzten alle Gruppen direkt die HDM fort, so wie es 
die Leitlinien erfordern. Leider ließen sich die Artefakte, die die HDM normalerweise auf das EKG hat von der Puppe nicht simulieren und so war unter laufender HDM der Sinusrhythmus sichtbar, wenn die EKG-Elektroden angeschlossen waren. Ca. 50\% beider Gruppen brachen die HDM zu früh ab, obwohl die Leitlinien eine durchgehende HDM für zwei Minuten nach Rhythmuskonversion fordern. Eine Erklärung hierfür könnte ein Fixierungsfehler auf den Monitor mit dem neuen Rhythmus sein, in dessen Analyse sich auch der Teamleiter miteinbinden ließ und dieser dadurch nicht mehr auf die Zeit achtete. Es könnte auch sein, dass die Studierenden Sorge hatten, dem „Patienten“ zu schaden, wenn sie bei sichtbarem Sinusrhythmus die Herzdruckmassage fortführten. In einem Bericht über die Umsetzung der Leitlinien 2005 im medizinischen Curriculum wird berichtet, dass Studierende Schwierigkeiten mit der Fortsetzung der Reanimation haben, wenn die vorangegangene Defibrillation mit einer Rhythmuskonversion einhergeht (Roessler et al. 2008).

Ähnlich wie der gestörte EKG-Rhythmus durch HDM, lassen sich auch mögliche reversible Ursachen eines Kreislaufstillstands, wie z.B. Hypothermie, nicht simulieren und werden deshalb vielleicht meist nicht bedacht und vergessen zu diskutieren.

Zwar werden Simulationen immer so realitätsgetreu wie möglich inszeniert, jedoch lässt sich vermuten, dass wahrscheinlich die Qualität der Performance auch ein stückweit davon abhängt wie gut sich jeder einzelne Proband auf die Situation einlässt und ,mitspielt““. Letztendlich bleibt die Simulation nur ein Abbild der Wirklichkeit.

\subsection{Reanimationsskills}

Marsch und Mitarbeiter konnten zeigen, dass ein Reanimationsversagen in den ersten Minuten nach Eintreten von Kammerflimmern mit reduzierter Interaktion im Team und weniger Kommunikation assoziiert ist (Marsch et al. 2004). Reanimationsversagen wurde hierbei u.a. definiert über eine NFT größer als 15 Sekunden in den ersten fünf min, wobei die Defibrillationszeit nicht inbegriffen war. Jedoch gibt es auch gegensätzliche Studien. Hänsel et al zeigte, dass weder CRM-Training noch Simulatortraining die klinische Performance von Studierenden in einem Szenario mit Sepsis verbessert (Hansel et al. 2012). Da in dieser Studie nicht mit Lehrvideos gearbeitet wurde, um den Studierenden CRM zu veranschaulichen, besteht die Möglichkeit, dass die Vermittlung der CRM- 
Kriterien für die Studierenden zu abstrakt schien und sie diese nicht gut genug in die Praxis übersetzen konnten. Auch die vorliegenden Ergebnisse scheinen auf einen positiven Zusammenhang zwischen CRM-Training und Reanimationsqualität hinzuweisen.

\subsubsection{NFT}

Die Unterbrechung von Herzdruckmassage während CPR, wird als no-flow time oder hands-off time bezeichnet. Die große Bedeutung der Reduzierung von NFT wird häufig in der Literatur erwähnt und gilt als zentraler Parameter, um Reanimationsqualität zu messen (Hunziker et al. 2009; Mondrup et al. 2011). Auch in der vorliegenden Arbeit gehört die NFT zu einem der Hauptuntersuchungspunkte im Zusammenhang mit dem Effekt von CRM-Training. Die CRM-geschulten Gruppen wiesen eine signifikant kürzere NFT auf als

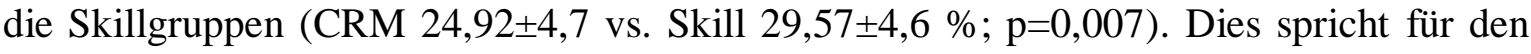
positiven Einfluss von CRM auf die NFT. Geregeltere Arbeitsabläufe durch mehr Antizipation und Planung, bessere Kommunikation über direkte Aufforderungen durch den Teamleiter und Rückmeldungen von den Teammitgliedern, Reevaluation der bisherigen Handlungen, effektives Teamwork und Aufgabenverteilung durch Nutzung von Resourcen. All das könnten die Gründe für eine verkürzte NFT sein. Dieser Zusammenhang hat klinisch eine sehr große Relevanz, denn eine ununterbrochene Herzdruckmassage ermöglicht eine gewisse Kreislaufaktivität, die unabdingbar ist für die CPR-Effektivität und somit für das Überleben der Patienten (Valenzuela et al. 2005). Eine dänische Studie identifizierte unerfahrene Teamleiter, Überlastung der Teammitglieder mit Aufgaben und das Unvermögen, sich vorrangig auf die Herzdruckmassage $\mathrm{zu}$ konzentrieren als Schwachpunkte eines Reanimationsteams und forderte das fokussierte Reanimationstraining in diesen nicht-technischen Fähigkeiten, um die NFT zu reduzieren (Andersen et al. 2010). Die oben erwähnte Studie von Hunziker et al. zeigte die Verminderung von NFT durch Teams, die vor der Reanimation die Möglichkeit hatten, sich dem Prozess der Teambildung zu unterziehen (Hunziker et al. 2009). Schon früher wurde die Effektivität von Teamtraining im ALS vermutet und testweise ein Teamführungsseminar in ALSProvider-Kurse des ERC integriert. Das Resultat zeigte tatsächlich ein erhöhtes Vorkommen von Teamführungsverhalten in der Versuchsgruppe, und es wurde die Integration solch eines Seminars in alle ALS-Provider-Kurse des ERC gefordert (Cooper 2001). Interessant im Zusammenhang mit der NFT ist auch die Untersuchung der 
Aktivitäten während der Unterbrechungen der Herzdruckmassage. Sie zeigte, dass sich bei 56\% der NFT alle Teammitglieder auf die gleiche sekundäre Aktivität konzentrieren wie das Fokussieren auf den Monitor, den Defibrillator und die Vitalparameter (Tschan et al. 2011). Dies ist ein weiterer Hinweis auf die Wichtigkeit eines Teamleiters, der sich nicht aktiv handelnd in den Reanimationsvorgang einbringen sollte, sondern nur delegierend, außer eingeschränkte Ressourcen lassen dies nicht anders zu. Die unnötigen Unterbrechungen der Herzdruckmassage wurden in einer anderen Studie im Verhältnis zu der möglichen Unterbrechungszeit (Start HDM bis ROSC) gemessen. Sie zeigte, dass die Zeit, in der die HDM unnötig unterbrochen wurde, 10-16\% der gesamt möglichen Unterbrechungszeit ausmachte und dass sich die Probanden dieser unnötigen Unterbrechungen während des Szenarios auch gar nicht bewusst waren (Marsch et al. 2005b). Hier lagen wahrscheinlich auch Fixierungsfehler der Teammitglieder vor, die zu den Unterbrechungen führten.

Aus diesen Erkenntnissen lässt sich schließen, dass die Einführung von CRM-Training in die Ausbildung von ALS bereits innerhalb des medizinischen Curriculums sinnvoll erscheint, um dem Ziel der Leitlinien 2005, also der ununterbrochenen Herzdruckmassage, in der Praxis näher zu kommen. Die nicht-technischen Fähigkeiten des CRM hat auch schon in die ERC-Leitlinien 2010 Einzug gehalten (Soar et al. 2010). Die Implementierung von CRM-Training in das Curriculum der Notfallmedizin während des Studiums sollte in allen Medizinischen Fakultäten forciert werden, um die Studierenden effektiver in CPR ausbilden zu können. Ob die Einführung von CRM auch in anderen Zusammenhängen und Fachdisziplinen des medizinischen Curriculums sinnvoll ist, bleibt zu untersuchen.

\subsubsection{Herzdruckmassage}

In den Daten der Herzdruckmassage ergaben sich keine signifikanten Unterschiede (Tab. 3.3.2). Die Druckfrequenz beider Gruppen lag zwischen 105-110 Kompressionen/min, also relativ nah an den geforderten 100/min in den Leitlinien 2005 (Handley et al. 2005). In Studien zeigte sich ein deutlicher Einfluss der Frequenz auf das Überleben. Patienten, die mit einer Frequenz von durchschnittlich 90/min reanimiert wurden, wiesen eine höhere Überlebensrate auf, als Patienten, die nur mit einer Frequenz von 79/min reanimiert wurden (Abella et al. 2005). Die Qualität der Kompressionen ist mit der Druckfrequenz assoziiert. Field et al. zeigten, dass eine Frequenz größer als 120/min signifikant die 
Qualität (Drucktiefe und Entlastung) der Kompressionen beeinträchtigt und eine Frequenz von 100-120/min optimal sei (Field et al. 2012). Auch durch den BMI und die Fitness der Helfer kann die Qualität der Herzdruckmassage vorausgesagt werden (Russo et al. 2011).

\subsubsection{Maskenbeatmung}

Auch hinsichtlich der Maskenbeatmung ergaben sich keine Unterschiede zwischen den Gruppen (Tab 3.3.3). Die CRM-Gruppen führten zu 61\% korrekte Beatmungen durch und die Skillgruppen zu 52\%. Dies spiegelt den derzeitigen Trend in der Notfallmedizin wider, die Maskenbeatmung zugunsten der Einlage eines supraglottischen Atemweges zu verlassen (Heuer et al. 2010). In der Literatur finden sich aber auch hohe Erfolgsraten hinsichtlich der Maskenbeatmung: Studentische Probanden, ohne Routine im Airwaymanagement, ventilierten unter Supervision $\mathrm{zu}$ ca. $86 \%$ effizient über eine Gesichtsmaske (Timmermann et al. 2007). Eine Erklärung für diese Differenz in den Leistungen der Studierenden könnte sein, dass im CAST ein höherer Erfolgsdruck und erschwerte Bedingungen herrschten als im OP unter Supervision. Dessen ungeachtet sollte die Maskenbeatmung vermehrt im Studium geübt werden, da sie zu den Basisfertigkeiten eines jeden Arztes gehört.

\subsection{Limitationen}

Die Hypothese, dass CRM-Schulungen einen positiven Einfluss auf die NFT und Algorithmuskonformität während der CPR ausüben, lässt sich unter der Berücksichtigung der Limitationen im Studienaufbau verifizieren. Der Dozent, der das CAST betreute, war nicht bei allen Gruppen derselbe, da mehrere CASTs zur gleichen Zeit in benachbarten Räumen stattfanden. Aus zeitlichen und organisatorischen Gründen war dies nicht anders möglich. Da alle Dozenten aber einheitliche Instruktionen vor dem CAST erhielten und die Interaktion zwischen der Gruppe und dem Dozenten vor dem Messzeitraum minimal war (ca. 5min), wurde dieser Störfaktor minimiert. Ähnlich verhielt es sich im CRM-Seminar. Auch dieses konnte nicht immer von dem gleichen Dozenten gehalten werden, aber durch identische Präsentationsfolien (siehe Anhang 6.1) wurde wahrscheinlich eine große Übereinstimmung im Ablauf geschaffen. Kleinere Abweichungen im Ablauf des CASTs und des CRM-Seminars konnten also durch die Dozenteninhomogenität nicht ausgeschlossen werden. Desweiteren ist es möglich, dass Studierende nach Besuch des 
CRM-Seminars über dessen Inhalt ihren Kommilitonen berichteten, die der anderen Kohorte angehörten und diese das überlieferte CRM-Verhalten im CAST gezeigt haben könnten. Da für die Skillgruppen das Seminar jedoch nach dem CAST auch noch auf dem Stundenplan stand, ist eine detaillierte Weitergabe der Informationen, auch im Anbetracht der vorliegenden Ergebnisse, unwahrscheinlich.

Als Schwachpunkte der Messungen über den PC-Skillreport ist die Unzuverlässigkeit der Puppe zu nennen. Es gab häufig Schwierigkeiten, da die Puppen sich gegenseitig in ihren eingestellten EKG-Rhythmen zu stören schienen, wenn mehrere Szenarien in benachbarten Räumen gleichzeitig stattfanden, die Puppe abstürzte oder das Programm die Reanimationsdaten nicht aufzeichnete. Entsprechende Szenarien konnten deshalb nicht gewertet werden.

Die Auswertung der Daten und Videos wurde aufgrund des zeitlich hohen Aufwandes nur von einer Person durchgeführt, jedoch bekam diese erst nach der Auswertung die Informationen über die Kohortenzugehörigkeit der einzelnen Gruppen, sodass eine objektive Beurteilung möglich war. 


\section{ZUSAMMENFASSUNG}

Crisis Resource Management (CRM) hat seinen Ursprung in der Luftfahrt und zielt auf die Schulung in zwischenmenschlichen Fähigkeiten ab, den sogenannten non-technical skills, wie Kommunikation, Teambildung/-leitung, Antizipation und Planung, Nutzung von Resourcen und Reevaluation. Auch in der Medizin wird das CRM-Training genutzt, um die Effektivität von Teams in Krisensituationen, wie z.B. der kardiopulmonalen Reanimation, zu steigern und somit das Outcome der Patienten zu verbessern. Jedoch ist die Integration von CRM in das medizinische Curriculum bislang nur an wenigen Fakultäten Deutschlands erfolgt. Am Universitätsklinikum Göttingen wurde ein CRMSeminar in das Curriculum des Querschnittsbereichs Notfallmedizin und Intensivmedizin aufgenommen. Das Ziel der vorliegenden Arbeit war zu überprüfen, ob sich das CRMSeminar mit den dort gelehrten Verhaltensstrategien auf die Qualität der CPR auswirkt und ob sich die Studierenden dadurch besser an den ALS-Algorithmus halten. Ein „Cardiac Arrest Scenario Training" (CAST), also ein Praktikum, in dem ALS an einer Simulationspuppe im Team geübt wurde, diente der Datenerhebung. Die Daten wurden teils über die Simulationspuppe detektiert (PC-Skillreporting System@), teils einem parallel aufgenommenen Video entnommen. Anhand des Videos und eines entwickelten Berwertungsscores wurde die Konformität zum ALS-Algorithmus (ERC 2005) mit Punkten bewertet. Eine Kohorte nahm vor dem CAST am CRM-Seminar teil (CRMGruppen), die andere hinterher (Skillgruppen). Letztere durften vorher jedoch ein Skillseminar besuchen, in dem alle praktischen Fähigkeiten wie Airwaymanagement, BLS, Defibrillation und Rhythmusanalyse wiederholt und geübt wurden, das den CRM-Gruppen wiederum erst nach dem CAST angeboten wurde.

Die CRM-Gruppen hielten sich im CAST signifikant enger an den ALS-Algorithmus (CRM 37 Punkte, Min/Max 24/57 Punkte vs. Skill 29 Punkte, Min/Max 15/42 Punkte; $\mathrm{p}=0,005)$, vor allem in den Untergruppen „Medikamente“ und „Intubation“. Die CRMGruppen hatten außerdem eine signifikant geringere NFT als die Skillgruppen (CRM

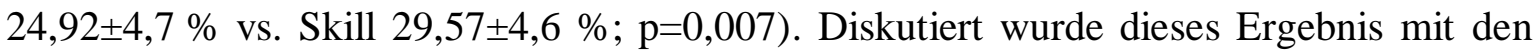
möglichen positiven Einflüssen des vermehrten CRM-konformen Verhaltens innerhalb der CRM-Gruppen auf die genannten Parameter. Eine parallel stattfindende kommunikationspsychologische Untersuchung zeigte außerdem mehr Teamleiter- 
Verbalisationen in den Kategorien direkte und indirekte Aufforderungen, Aufgabenverteilung und Planung in den CRM-Gruppen.

Es konnte gezeigt werden, dass ein CRM-Seminar für Medizinstudenten im letzten Jahr zu einer signifikanten Verminderung der NFT während CPR führte und sich die Studierenden außerdem enger an den ALS-Algorithmus des ERC hielten. Die Implementierung eines solchen Seminars in das medizinische Curriculum kann mit den vorliegenden Ergebnissen befürwortet werden. 
6. ANHANG

\title{
6.1 Präsentationsfolien des CRM-Seminars
}

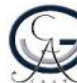

\author{
UNIVERSITÄTSMEDIZIN "
GÖTtIINGEN " $\mathbf{M G}$
}

Inhalt des Seminars

Modul 6.2

Notfall- und Intensivmedizin

- ALS Wiederholung

Die Bewältigung von Zwischenfällen

- Der „Human Factor" -

Sinnvolle Aufteilung zur Teamreanimation

- Zwischenfälle in der Medizin

- Bedeutung der Human Factors

CRM Prinzipien

- Videoanalyse

Zusammenfassung

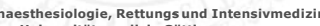

Universitätsmedizizing G̈ttingen
www.zarionline.de

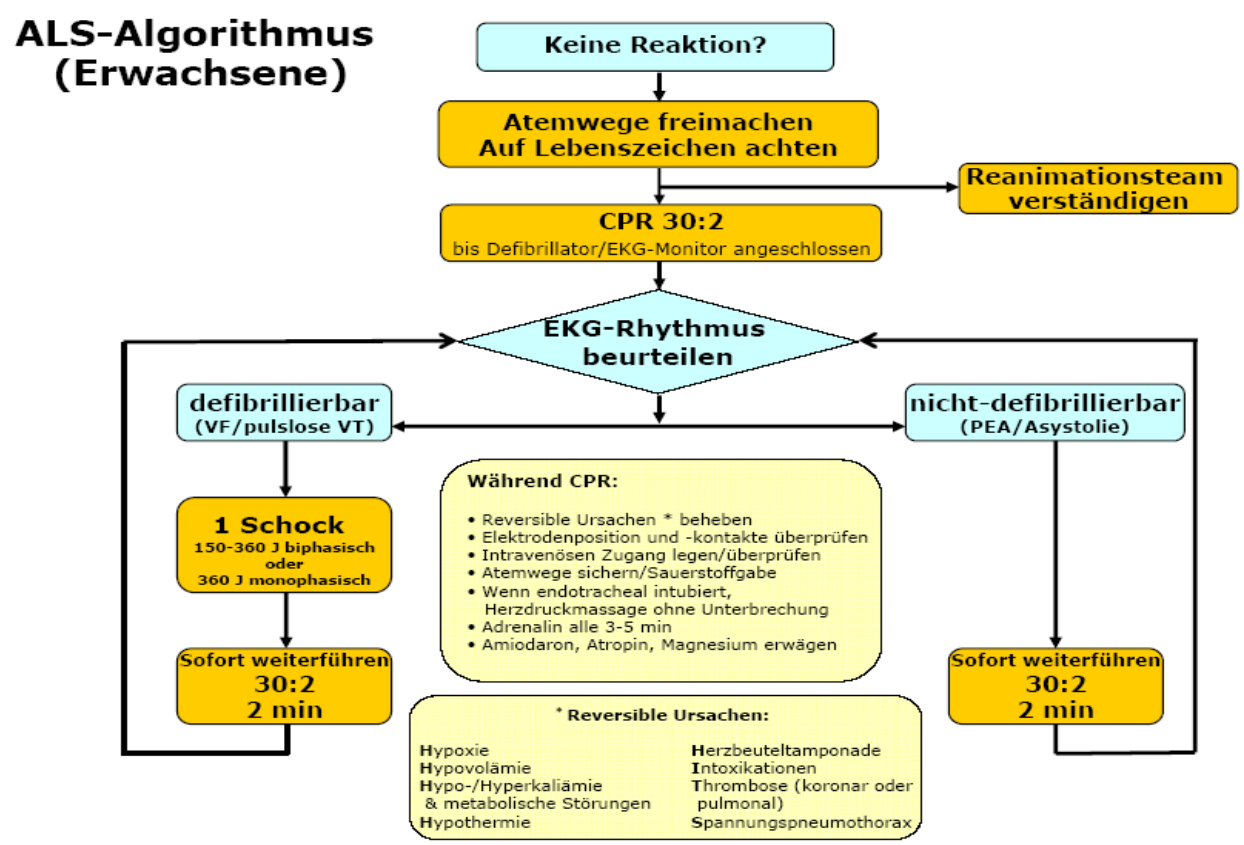

FILM: Schwarzwaldklinik Aufgabe: Welche Fehler werden gemacht

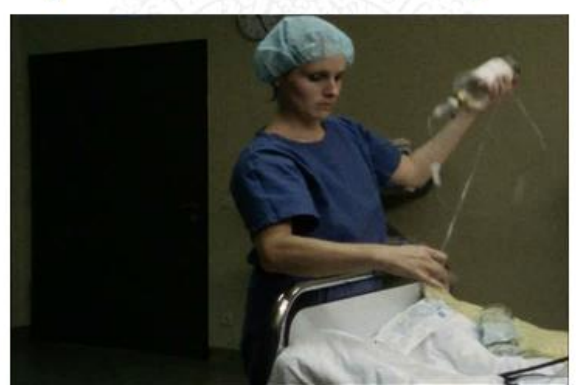

Organisieren Sie Ihr ALS - Team

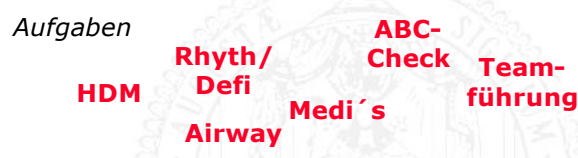
Helfen

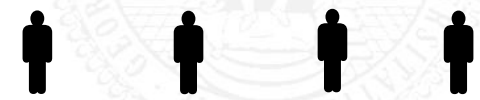

Teammitglieder 


\section{Herz-Druck-Massage(HDM)}

In meiner Funktion...

- suche ich den korrekten Druckpunkt und beginne sofort mit der Herzdruckmassage (HDM)

- komprimiere ich mit $100 / \mathrm{min}$ - mit einer Tiefe von 4-5cm

- mit einer vollständigen Entlastung

- mit 30:2 während der Atemweg noch nicht gesichert ist

- asynchron, wenn der Atemweg gesichert is

- achte ich auf minimale „Hands-off" Zeiten

- auf Fortführung der HDM während der Intubation

- auf Fortführung der HDM nach der Defibrillation ohne

Rhythmus-Kontrolle für weitere 2 Minuten

- gebe ich Rückmeldung an den Teamleader!

\section{Aufgabe: Airway}

In meiner Funktion ....

- führe ich den initialen Basischeck durch

- nehme mir Maske/Beutel/ $\mathrm{O}_{2}$ und

ventiliere ich $2 x$ nach 30 Kompressionen mit ca. $500 \mathrm{~m}$

- lasse ich mir die Intubation vorbereiten

- intubiere ich während der HDM unter laufender HDM

- kontrolliere ich die Tubuslage ( $\mathrm{CO}_{2}$, Auskultation)

- führe ich die MB nach Fehlintubation fort

- beatme ich nach korrekter Intubation mit einer Frequenz von $10-12 / \mathrm{min}$

- gebe ich Rückmeldung an den Teamleader!

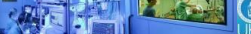

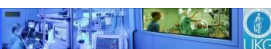

\section{Aufgabe: Defi/Rhythmus}

In meiner Funktion...

- führe ich den initialen Rhythmuscheck durch (mit Defipaddels)

- führe ggf. die sichere Frühdefibrillation mit $360 J$ (monophasisch) durch

- lege ich das EKG-Kabel an

- führe ich alle $\mathbf{2}$ min eine Rhythmuskontrolle und ggf. eine Defibrillation durch

- gebe ich Rückmeldung an den Teamleader!!!

\section{Aufgabe: Medis}

In meiner Funktion...

- lege ich einen iv-Zugang und hänge eine Infusion an

- ziehe ich 1 mg Adrenalin auf und gebe es vor dem 3. Schock

- ziehe ich $\mathbf{3 0 0} \mathbf{~ m g ~ A m i o d a r o n ~ a u f ~ u n d ~ g e b e ~ e s ~ v o r ~}$ dem 4. Schock

- gebe ich repetitiv $1 \mathrm{mg}$ Adrenalin alle 3-5 Minuten

- gebe ich Rückmeldung an den Teamleader!!!

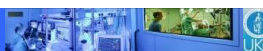

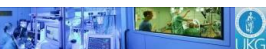

\section{Aufgabe: Hilfe}

In meiner Funktion...

- bereite ich die Intubation vor und helfe bei der Durchführung

- löst bei der HDM ab

- hält sich für weitere Assistenz bereit

- gebe ich Rückmeldung an den Teamleader!!!

\section{ALS - Teamorganisation}

\begin{tabular}{|c|c|c|c|}
\hline Aufgaben & Rhyth / & $\begin{array}{l}\text { ABC- } \\
\text { Check }\end{array}$ & Team- \\
\hline 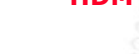 & & Medi's & \\
\hline
\end{tabular}

Helfen

Teammitglieder

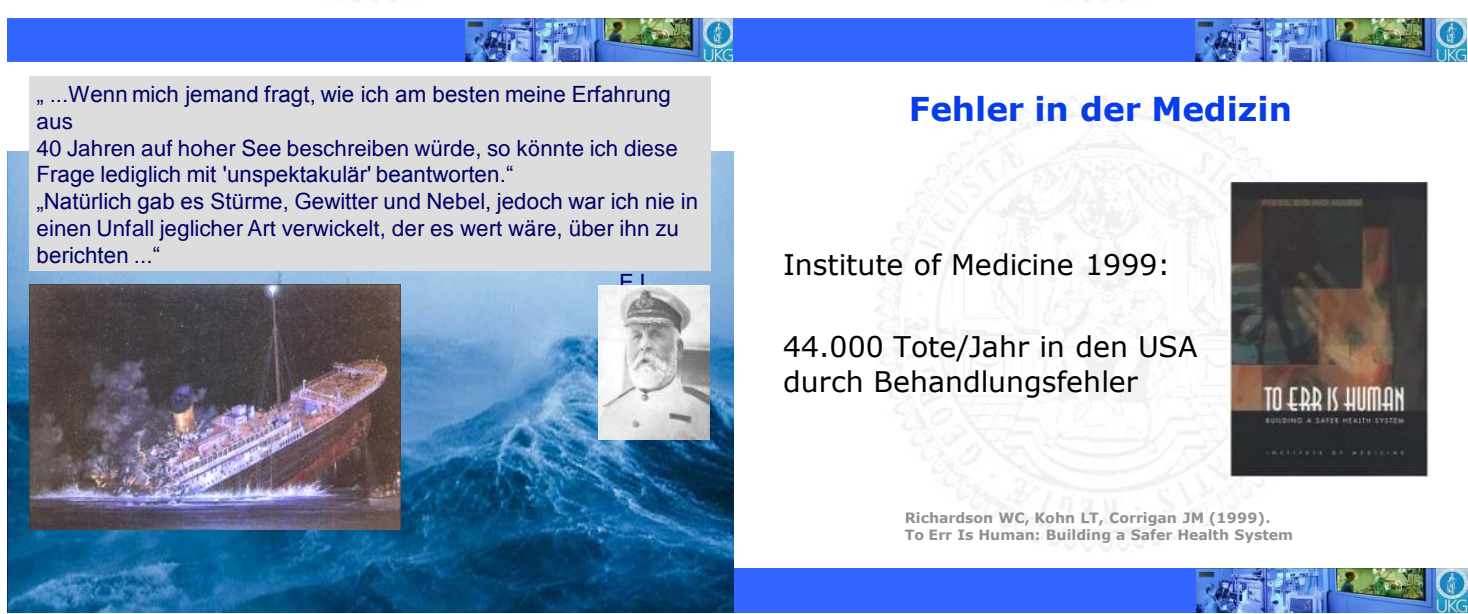




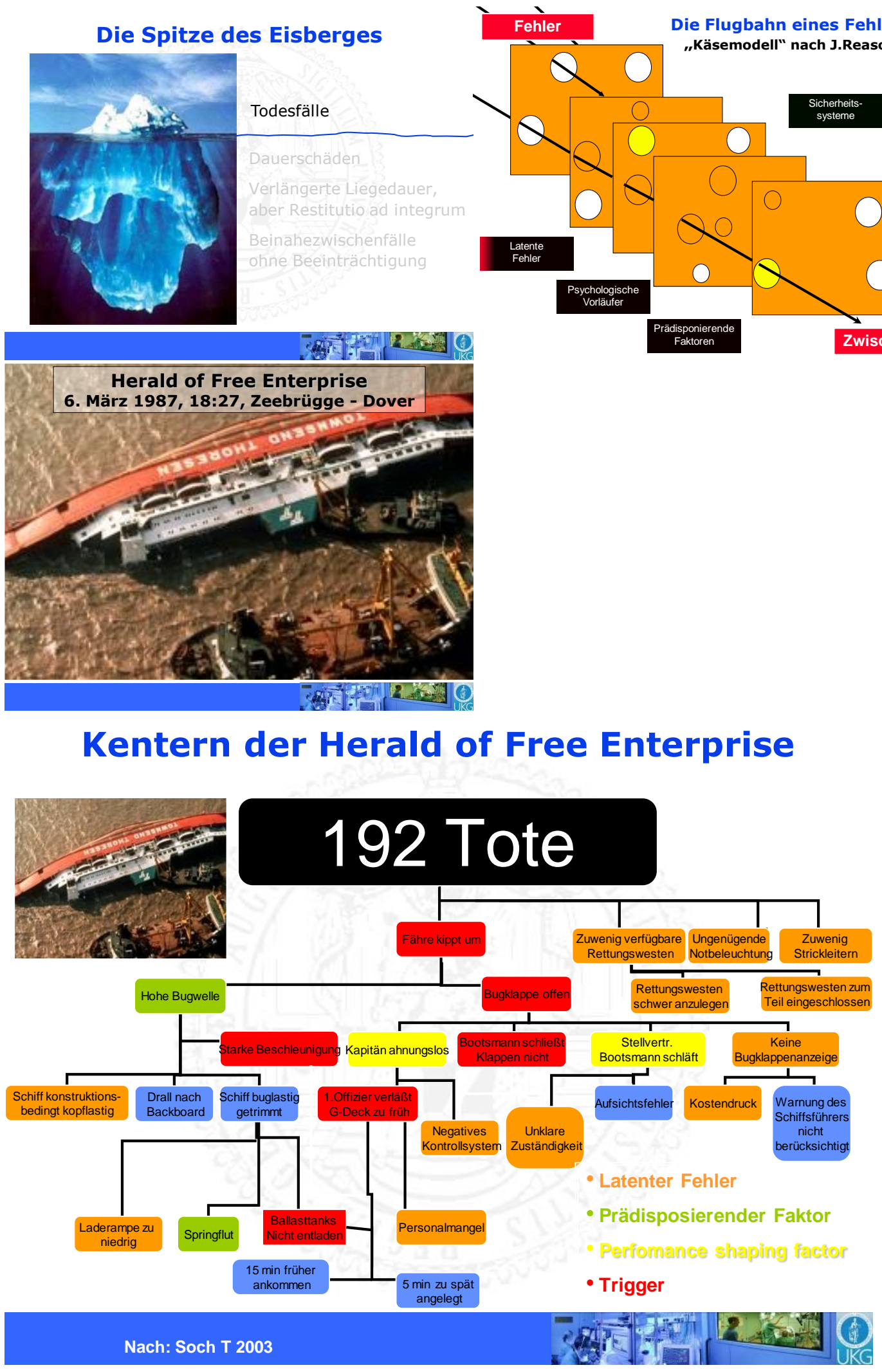


Aufgabe:

„Human Factor"

Formulieren Sie Fertigkeiten, die eine gute Ärztin/einen guten Arzt in einer Zwischenfallssituation ausmachen!
Der „Faktor Mensch":

Sammelbegriff für psychische, kognitive und soziale Einflussfaktoren in soziotechnischen Systemen

Ursache für $\approx \mathbf{8 0} \%$ aller Zwischenfälle in der Medizin ${ }^{1}$

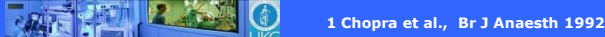

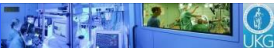

Wie löse ich den Notfall ?

Kontrollebenen des Handels

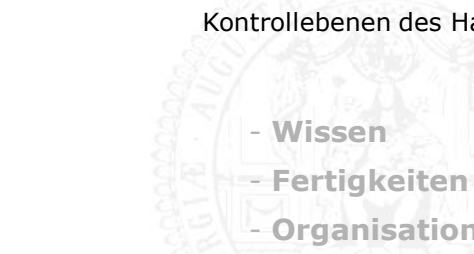

Das Pyramidenmodell der klinischen Kompetenz

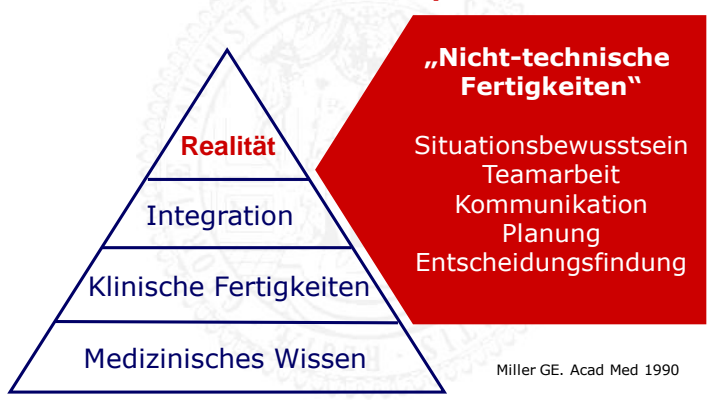

Crisis Resource Management (CRM)

$=$ Zwischenfallmanagement

$$
\text { CRM - Prinzipien }
$$
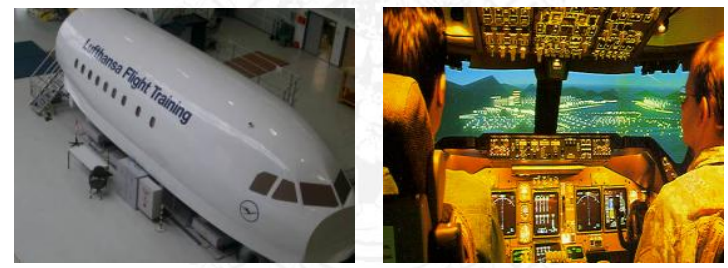

1. Antizipation und Planung

2. Teambildung und -führung

3. Kommunikation

4. Nutzung von Ressourcen

5. Re-Evaluation

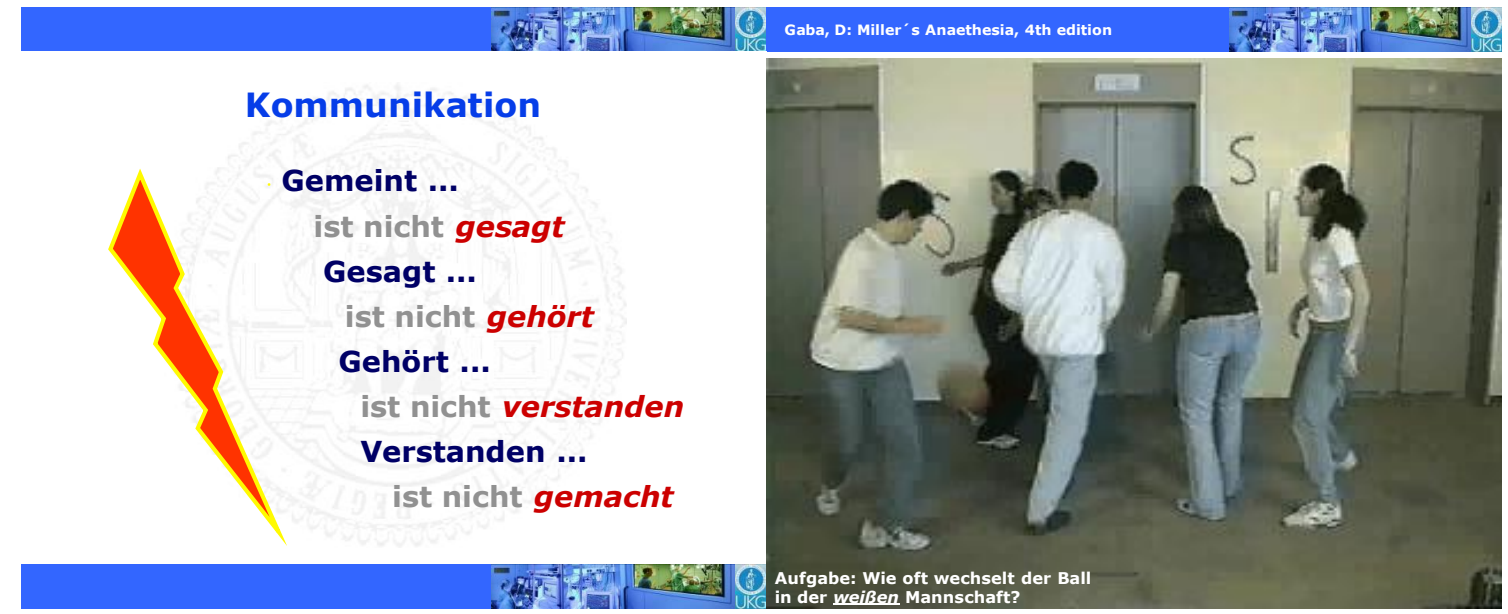


Video 1/Aufgabe:

Beobachten Sie Maßnahmen der CPR

- Welche Aufgaben wurden gut/schlecht gelöst?

- Überlegen Sie warum? Erkennen sie Beispiele für gutes oder schlechtes CRM ?
Video 2/Aufgabe:

Beobachten Sie Maßnahmen der CPR

- Welche Aufgaben wurden gut/schlecht gelöst?

- Überlegen Sie warum? Erkennen sie Beispiele für gutes oder schlechtes CRM ?

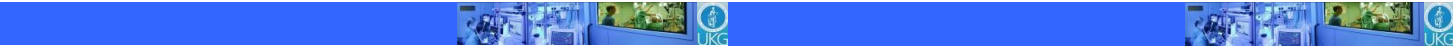 \\ Video 3 / Aufgabe: CRM BINGO \\ Zusammenfassung I \\ Beobachten Sie Maßnahmen der CPR \\ - Folgen Sie den Anweisungen der Bingo Karten! \\ Zwischenfälle in der Medizin \\ treten häufig auf \\ entstehen aus einer Verkettung von Ereignissen \\ basieren meist auf dem „Human Factor"}

\section{Zusammenfassung II}

Gutes Zwischenfallsmanagement

bedeutet effektive/s

Antizipation und Planung

Teamarbeit und Führung

Kommunikation

Nutzung von Ressourcen

Re-Evaluation

\section{Zusammenfassung III}

Eckpfeiler des Adavance Life Support

1. Team organisieren

2. „Push fast $(100 / \mathrm{min})$

Push hard (4-5cm)

Don' $\mathrm{t}$ interrupt"

3. alle $\mathbf{2}$ min Rhythmuskontrolle, ggf. Defibrillation

4. Effektive Ventilation

5. iv-Zugang, Adrenalin, Atropin und Amiodaron nach Algorithmus 


\section{LITERATURVERZEICHNIS}

Abella BS, Sandbo N, Vassilatos P, Alvarado JP, O'Hearn N, Wigder HN, Hoffman P, Tynus K, Vanden Hoek TL, Becker LB (2005): Chest compression rates during cardiopulmonary resuscitation are suboptimal: a prospective study during inhospital cardiac arrest. Circulation $\underline{111}$ (4), 428-434

Andersen PO, Jensen MK, Lippert A, Ostergaard D (2010): Identifying non-technical skills and barriers for improvement of teamwork in cardiac arrest teams. Resuscitation $\underline{81}$ (6), 695-702

Billings CE, Reynard WD (1984): Human factors in aircraft incidents: results of a 7-year study. Aviat Space Environ Med 55 (10), 960-965

Capella J, Smith S, Philp A, Putnam T, Gilbert C, Fry W, Harvey E, Wright A, Henderson K, Baker D, Ranson S, Remine S (2010): Teamwork training improves the clinical care of trauma patients. J Surg Educ 67 (6), 439-443

Chandra DB, Savoldelli GL, Joo HS, Weiss ID, Naik VN (2008): Fiberoptic oral intubation: the effect of model fidelity on training for transfer to patient care. Anesthesiology 109 (6), 1007-1013

Chen L, Hsiao AL (2008): Randomized trial of endotracheal tube versus laryngeal mask airway in simulated prehospital pediatric arrest. Pediatrics 122 (2), 294-297

Chopra V, Bovill JG, Spierdijk J, Koornneef F (1992): Reported significant observations during anaesthesia: a prospective analysis over an 18-month period. Br J Anaesth $\underline{68}(1), 13-17$

Cooper S (2001): Developing leaders for advanced life support: evaluation of a training programme. Resuscitation $\underline{49}$ (1), 33-38

Crile G, Dolley DH (1906): An Experimental Research into the Resuscitation of Dogs Killed by Anesthetics and Asphyxia. J Exp Med $\underline{8}$ (6), 713-725

Fernandez Castelao E, Russo SG, Cremer S, Strack M, Kaminski L, Eich C, Timmermann A, Boos M (2011): Positive impact of crisis resource management training on noflow time and team member verbalisations during simulated cardiopulmonary resuscitation: a randomised controlled trial. Resuscitation $\underline{82}$ (10), 1338-1343

Field RA, Soar J, Davies RP, Akhtar N, Perkins GD (2012): The impact of chest compression rates on quality of chest compressions - a manikin study. Resuscitation 83 (3), 360-364

Flanagan B, Nestel D, Joseph M (2004): Making patient safety the focus: crisis resource management in the undergraduate curriculum. Med Educ $\underline{38}$ (1), 56-66

Gaba DM, Howard SK, Fish KJ, Smith BE, Sowb YA (2001): Simulation-Based Training in Anesthesia Crisis Resource Management (ACRM): A Decade of Experience. Simulation \& Gaming 32 (2), 175-193

Gallagher EJ, Lombardi G, Gennis P (1995): Effectiveness of bystander cardiopulmonary resuscitation and survival following out-of-hospital cardiac arrest. Jama 274 (24), 1922-1925 
Greene DG, Bauer RO, Janney CD, Elam JO (1957): Expired air resuscitation in paralyzed human subjects. J Appl Physiol 11 (2), 313-318

Handley AJ, Koster R, Monsieurs K, Perkins GD, Davies S, Bossaert L (2005): European Resuscitation Council guidelines for resuscitation 2005. Section 2. Adult basic life support and use of automated external defibrillators. Resuscitation 67 Suppl 1 7-23

Hansel M, Winkelmann AM, Hardt F, Gijselaers W, Hacker W, Stiehl M, Koch T, Muller MP (2012): Impact of simulator training and crew resource management training on final-year medical Studierendes' performance in sepsis resuscitation: a randomized trial. Minerva Anestesiol 78 (8), 901-9

Harris D, Li WC (2008): Cockpit design and cross-cultural issues underlying failures in crew resource management. Aviat Space Environ Med 79 (5), 537-538

Helmreich RL (2000): On error management: lessons from aviation. Bmj 320 (7237), 781785

Heuer JF, Barwing J, Eich C, Quintel M, Crozier TA, Roessler M (2010): Initial ventilation through laryngeal tube instead of face mask in out-of-hospital cardiopulmonary arrest is effective and safe. Eur J Emerg Med 17 (1), 10-5

Howard SK, Gaba DM, Fish KJ, Yang G, Sarnquist FH (1992): Anesthesia crisis resource management training: teaching anesthesiologists to handle critical incidents. Aviat Space Environ Med $\underline{63}$ (9), 763-770

Hunt EA, Shilkofski NA, Stavroudis TA, Nelson KL (2007): Simulation: translation to improved team performance. Anesthesiol Clin $\underline{25}$ (2), 301-319

Hunziker S, Tschan F, Semmer NK, Zobrist R, Spychiger M, Breuer M, Hunziker PR, Marsch SC (2009): Hands-on time during cardiopulmonary resuscitation is affected by the process of teambuilding: a prospective randomised simulator-based trial. BMC Emerg Med 9 (3),

Ilper H, Kunz T, Pfleger H, Schalk R, Byhahn C, Ackermann H, Breitkreutz R (2012): Comparative quality analysis of hands-off time in simulated basic and advanced life support following European Resuscitation Council 2000 and 2005 guidelines. Emerg Med J $\underline{29}$ (2), 95-99

Kohn LT, Corrigan JM, Donaldson MS: To err is human: building a safer health system. National Academies Press, Washington D.C. 2000

Kouwenhoven WB, Jude JR, Knickerbocker GG (1960): Closed-chest cardiac massage. Jama 173 1064-1067

Krüger A, Gillmann B, Hardt C, Doring R, Beckers SK, Rossaint R (2009): [Teaching non-technical skills for critical incidents: Crisis resource management training for medical Studierendes]. Anaesthesist $\underline{58}$ (6), 582-8

Kurrek MM, Fish KJ (1996): Anaesthesia crisis resource management training: an intimidating concept, a rewarding experience. Can J Anaesth $\underline{43}$ (5 Pt 1), 430-434

Leblanc VR (2011): Review article: simulation in anesthesia: state of the science and looking forward. Can J Anaesth $\underline{59}$ (2), 193-202

Lerner S, Magrane D, Friedman E (2009): Teaching teamwork in medical education. Mt Sinai J Med 76 (4), 318-329 
Liss HP (1986): A history of resuscitation. Ann Emerg Med 15 (1), 65-72

Luscher F, Hunziker S, Gaillard V, Tschan F, Semmer NK, Hunziker PR, Marsch S (2010): Proficiency in cardiopulmonary resuscitation of medical Studierendes at graduation: a simulator-based comparison with general practitioners. Swiss Med Wkly $\underline{140}$ (3-4), 57-61

Marsch SC, Muller C, Marquardt K, Conrad G, Tschan F, Hunziker PR (2004): Human factors affect the quality of cardiopulmonary resuscitation in simulated cardiac arrests. Resuscitation $\underline{60}$ (1), 51-56

Marsch SC, Tschan F, Semmer N, Spychiger M, Breuer M, Hunziker PR (2005a): Performance of first responders in simulated cardiac arrests. Crit Care Med 33 (5), 963-967

Marsch SC, Tschan F, Semmer N, Spychiger M, Breuer M, Hunziker PR (2005b): Unnecessary interruptions of cardiac massage during simulated cardiac arrests. Eur J Anaesthesiol 22 (11), 831-833

Mondrup F, Brabrand M, Folkestad L, Oxlund J, Wiborg KR, Sand NP, Knudsen T (2011): In-hospital resuscitation evaluated by in situ simulation: a prospective simulation study. Scand J Trauma Resusc Emerg Med 1955

Morey JC, Simon R, Jay GD, Wears RL, Salisbury M, Dukes KA, Berns SD (2002): Error reduction and performance improvement in the emergency department through formal teamwork training: evaluation results of the MedTeams project. Health Serv Res $\underline{37}$ (6), 1553-1581

Morrissey J (2004): Patient safety proves elusive. Five years after publication of the IOM's 'To Err is Human,' there's plenty of activity on patient safety, but progress is another matter. Mod Healthc $\underline{34}$ (44), 6-7, 24-5, 28-32

Nolan JP, Deakin CD, Soar J, Bottiger BW, Smith G (2005): European Resuscitation Council guidelines for resuscitation 2005. Section 4. Adult advanced life support. Resuscitation 67 Suppl 1 39-86

Okuda Y, Bryson EO, DeMaria S, Jr., Jacobson L, Quinones J, Shen B, Levine AI (2009): The utility of simulation in medical education: what is the evidence? Mt Sinai J Med $\underline{76}$ (4), 330-343

Olasveengen TM, Vik E, Kuzovlev A, Sunde K (2009): Effect of implementation of new resuscitation guidelines on quality of cardiopulmonary resuscitation and survival. Resuscitation $\underline{80}$ (4), 407-411

Paraskos JA (1993): History of CPR and the role of the national conference. Ann Emerg Med 22 (2), 275-280

Perkins GD, Davies RP, Stallard N, Bullock I, Stevens H, Lockey A (2007): Advanced life support cardiac arrest scenario test evaluation. Resuscitation 75 (3), 484-490

Rall M, Dieckmann P (2005): Safety culture and crisis resource management in airway management: general principles to enhance patient safety in critical airway situations. Best Pract Res Clin Anaesthesiol 19 (4), 539-557

Reiter DA, Strother CG, Weingart SD (2013): The quality of cardiopulmonary resuscitation using supraglottic airways and intraosseous devices: a simulation trial. Resuscitation $\underline{84}$ (1), 93-7 
Reznek M, Smith-Coggins R, Howard S, Kiran K, Harter P, Sowb Y, Gaba D, Krummel T (2003): Emergency medicine crisis resource management (EMCRM): pilot study of a simulation-based crisis management course for emergency medicine. Acad Emerg Med 10 (4), 386-389

Ricci MA, Brumsted JR (2012): Crew resource management: using aviation techniques to improve operating room safety. Aviat Space Environ Med 83 (4), 441-444

Risser DT, Rice MM, Salisbury ML, Simon R, Jay GD, Berns SD (1999): The potential for improved teamwork to reduce medical errors in the emergency department. The MedTeams Research Consortium. Ann Emerg Med 34 (3), 373-383

Roessler M, Lott C, Timmermann A, Russo S, Graf BM, Eich C (2008): Umsetzung der Reanimationsleitlinien 2005 in der Studierendeischen Lehre. Notfall+Rettungsmedizin 11 (2), 105-112

Ruesseler M, Weinlich M, Muller MP, Byhahn C, Marzi I, Walcher F (2012): Republished: Simulation training improves ability to manage medical emergencies. Postgrad Med J 88 (1040), 312-316

Russo SG, Neumann P, Reinhardt S, Timmermann A, Niklas A, Quintel M, Eich CB (2011): Impact of physical fitness and biometric data on the quality of external chest compression: a randomised, crossover trial. BMC Emerg Med 1120

Safar P (1999): Ventilation and cardiopulmonary resuscitation. Curr Opin Anaesthesiol 12 (2), 165-171

Schmidt CE, Hardt F, Moller J, Malchow B, Schmidt K, Bauer M (2010): [Improvement of team competence in the operating room : Training programs from aviation]. Anaesthesist $\underline{59}$ (8), 717-722, 724-726

Sica GT, Barron DM, Blum R, Frenna TH, Raemer DB (1999): Computerized realistic simulation: a teaching module for crisis management in radiology. AJR Am J Roentgenol $\underline{172}$ (2), 301-304

Soar J, Monsieurs KG, Ballance JH, Barelli A, Biarent D, Greif R, Handley AJ, Lockey AS, Richmond S, Ringsted C, Wyllie JP, Nolan JP, Perkins GD (2010): European Resuscitation Council Guidelines for Resuscitation 2010 Section 9. Principles of education in resuscitation. Resuscitation $\underline{81}$ (10), 1434-1444

Spreier SW, Fontaine MH, Malloy RL (2006): Leadership run amok. The destructive potential of overachievers. Harv Bus Rev $\underline{84}$ (6), 72-82, 144

Standards und Leitlinien:

(1974a): Standards for cardiopulmonary resuscitation (CPR) and emergency cardiac care (ECC). 3. Advanced life support. Jama 227 (7), Suppl:852-860

(1974b): Standards for cardiopulmonary resuscitation (CPR) and emergency cardiac care (ECC). II. Basic life support. Jama 227 (7), Suppl:841-851

(1980): Standards and guidelines for cardiopulmonary resuscitation (CPR) and emergency cardiac care (ECC). Jama $\underline{244}$ (5), 453-509 
(1986): Standards and guidelines for Cardiopulmonary Resuscitation (CPR) and Emergency Cardiac Care (ECC). National Academy of Sciences - National Research Council. Jama 255 (21), 2905-2989

(1992): Emergency Cardiac Care Committee and Subcommittees, American Heart Association. Jama $\underline{268}$ (16), 2171

Stelfox HT, Palmisani S, Scurlock C, Orav EJ, Bates DW (2006): The "To Err is Human" report and the patient safety literature. Qual Saf Health Care 15 (3), 174-178

Timmermann A, Eich C, Nickel E, Russo S, Barwing J, Heuer JF, Braun U (2005): [Simulation and airway management]. Anaesthesist 54 (6), 582-587

Timmermann A, Russo SG, Crozier TA, Eich C, Mundt B, Albrecht B, Graf BM (2007): Novices ventilate and intubate quicker and safer via intubating laryngeal mask than by conventional bag-mask ventilation and laryngoscopy. Anesthesiology 107 (4), 570-576

Tschan F, Vetterli M, Semmer NK, Hunziker S, Marsch SC (2011): Activities during interruptions in cardiopulmonary resuscitation: a simulator study. Resuscitation $\underline{82}$ (11), 1419-1423

Valenzuela TD, Kern KB, Clark LL, Berg RA, Berg MD, Berg DD, Hilwig RW, Otto CW, Newburn D, Ewy GA (2005): Interruptions of chest compressions during emergency medical systems resuscitation. Circulation 112 (9), 1259-1265

Volk MS, Ward J, Irias N, Navedo A, Pollart J, Weinstock PH (2011): Using medical simulation to teach crisis resource management and decision-making skills to otolaryngology housestaff. Otolaryngol Head Neck Surg $\underline{145}$ (1), 35-42

Wayne DB, Siddall VJ, Butter J, Fudala MJ, Wade LD, Feinglass J, McGaghie WC (2006): A longitudinal study of internal medicine residents' retention of advanced cardiac life support skills. Acad Med $\underline{81}$ (10 Suppl), 9-12

Weaver WD, Hill D, Fahrenbruch CE (1988): Use of the automatic external defibrillator in the management of out-of-hospital cardiac arrest. N Engl J Med $\underline{319}$ 661-666

Weller J, Robinson B, Larsen P, Caldwell C (2004): Simulation-based training to improve acute care skills in medical undergraduates. N Z Med J 117 (1204), 1119

Wenzel V, Russo S, Arntz HR, Bahr J, Baubin MA, Bottiger BW, Dirks B, Dorges V, Eich C, Fischer M, Wolcke B, Schwab S, Voelckel WG, Gervais HW (2006): [The new 2005 resuscitation guidelines of the European Resuscitation Council: comments and supplements]. Anaesthesist 55 (9), 958-966, 968-972, 974-979

Wik L, Steen PA, Bircher NG (1994): Quality of bystander cardiopulmonary resuscitation influences outcome after prehospital cardiac arrest. Resuscitation 28 (3), 195-203

Wik L, Kramer-Johansen J, Myklebust H, Sorebo H, Svensson L, Fellows B, Steen PA (2005): Quality of cardiopulmonary resuscitation during out-of-hospital cardiac arrest. Jama 293 (3), 299-304

Wilson KA, Burke CS, Priest HA, Salas E (2005): Promoting health care safety through training high reliability teams. Qual Saf Health Care 14 (4), 303-309 
Wischet W, Schusterschitz C (2009): Quality management and safety culture in medicine Do standard quality reports provide insights into the human factor of patient safety? Ger Med Sci 7 Doc30

Zoll PM, Linenthal AJ, Gibson W, Paul MH, Norman LR (1956): Termination of ventricular fibrillation in man by externally applied electric countershock. N Engl J Med 254 (16), 727-732 


\section{Danksagung}

Der erste Dank gilt meinem Doktorvater Herrn Prof. Dr. Arnd Timmermann für die freundliche Überlassung des Themas der Dissertation, für die Betreuung sowie die vielen produktiven Anregungen.

Besonderer Dank gilt auch Herrn Ezequiel Fernandez Castelao für die gute und nette Zusammenarbeit und die überragende Hilfsbereitschaft bei der Datenerhebung und Auswertung.

Weiterhin danke ich allen Mitarbeitern des Lehr- und Simulationszentrums sowie den beteiligten Personen aus der Abteilung für Sozial- und Kommunikationspsychologie der Georg-August-Universität in Göttingen. Im Einzelnen sind dies u.a. Stefan Cremer, Christoph Eich, Eike Nikkel, Sebastian Russo, Katrin Schwertfeger, Nils Jacobsen, Anita Konradi und Margarete Boos.

Danken möchte ich auch der Abteilung für Medizinische Statistik für die Beratung und Hilfe bei der Auswertung der Daten.

Mein herzlicher Dank gilt selbstverständlich auch allen teilnehmenden Studierenden der Humanmedizin sowie den helfenden Studierenden der Psychologie für die Ermöglichung der Datenerhebung für diese Arbeit. 